\title{
Photochemical Control of the Mechanical and Adhesive Properties of Crystalline Molecular Solids
}

Nicholas D. Blelloch, ${ }^{\dagger}$ Haydn T. Mitchell, ${ }^{\dagger}$ Louisa C. Greenburg, ${ }^{\dagger}$ Douglas W. Van Citters, ${ }^{\ddagger}$ and Katherine A. Mirica ${ }^{*}+$

+ Burke Laboratory, Department of Chemistry, Dartmouth College, Hanover, New Hampshire 03755, United States

\# Thayer School of Engineering, Dartmouth College, Hanover, New Hampshire 03755, United States

* Correspondence and requests should be addressed to K.A.M. (email: Katherine.A.Mirica@dartmouth.edu)

(52 pages)

\begin{tabular}{|c|c|c|}
\hline & Table of Contents & S1 \\
\hline SI. & General Materials & S2 \\
\hline SII. & Synthesis of Photoresponsive Crystalline Adhesives & S3 \\
\hline SIII. & NMR Spectroscopy & S5 \\
\hline SIV. & $\begin{array}{l}\text { Growing Single Crystals of Photoresponsive Crystalline } \\
\text { Adhesives }\end{array}$ & S14 \\
\hline SV. & $\begin{array}{l}\text { Crystal Structure Determination via Single Crystal X-Ray } \\
\text { Diffraction }\end{array}$ & S16 \\
\hline SVI. & Hirshfeld Surface Calculations and Fingerprint Plot Analysis & S22 \\
\hline SVII. & Calculation of Crystal Structure Correlation Coefficients & S23 \\
\hline SVIII. & $\begin{array}{l}\text { Melt-Bonding Quartz Substrates with Photoresponsive } \\
\text { Crystalline Adhesives }\end{array}$ & S25 \\
\hline SIX. & $\begin{array}{l}\text { Obtaining and Analyzing Micrographs of Polycrystalline Films } \\
\text { of Photoresponsive Adhesives }\end{array}$ & S26 \\
\hline SX. & Shear Testing of the Overlap Joint for Adhesive Measurements & S30 \\
\hline SXI. & $\begin{array}{l}\text { Powder X-Ray Diffraction and Calculation of Preferential } \\
\text { Orientation }\end{array}$ & S31 \\
\hline SXII. & UV/Vis Spectroscopy of Photoresponsive Crystalline Adhesives & S36 \\
\hline
\end{tabular}




\begin{tabular}{|c|l|c|}
\hline SXIII. & $\begin{array}{l}\text { Determination of the Reaction Rate for the Photolysis of } \\
\text { Photoresponsive Crystalline Adhesives }\end{array}$ & $\mathbf{S} 40$ \\
\hline SXIV. & $\begin{array}{l}\text { UV Irradiation of Quartz Assemblies Bonded with } \\
\text { Photoresponsive Crystalline Adhesives }\end{array}$ & $\mathrm{S} 42$ \\
\hline SXV. & $\begin{array}{l}\text { Solid-State Photolysis of Exposed Films of Photoresponsive } \\
\text { Crystalline Adhesives }\end{array}$ & $\mathrm{S} 45$ \\
\hline SXVI. & $\begin{array}{l}\text { Diffuse Reflectance Infrared Fourier Transform Spectroscopy } \\
\text { of Photoresponsive Crystalline Adhesives }\end{array}$ & $\mathbf{S 4 9}$ \\
\hline SXVII. & References & \\
\hline
\end{tabular}

\section{SI. General Materials}

All chemicals were purchased from commercial vendors and used as received: 2-nitrobenzyl alcohol (MilliporeSigma, Supplier No.: 8.820888.0025, CAS No.: 612-25-9), benzoyl chloride (Alfa Aesar, Supplier No.: A14107-30, CAS No.: 98-88-4), 1-naphthoyl chloride (Acros Organics, Supplier No.: AC27038-0100, CAS No.: 879-18-5), 2-naphthoyl chloride (TCl America, Supplier No.:N0048-25G, CAS No.: 2243-83-6), 1-adamantanecarbonyl chloride (Alfa Aesar, Supplier No.: L02352-09, CAS No.: 2094-72-6), benzoic acid (Acros Organics, Supplier No.: AC14913-5000, CAS No.: 65-85-0), 1-naphthoic acid (TCI America, Supplier No.: N0024-25G, CAS No.: 86-55-5), 2-naphthoic acid (Acros Organics, Supplier No.: AC17732-0250, CAS No.: 93-09-4), adamantane-1-carboxylic acid (Acros Organics, Supplier No.: AC14788-0050, CAS No.: 828-51-3), pyridine (VWR International, Supplier No.: BDH4562-1L, CAS No.: 110-8601), acetone-D6 (Cambridge Isotope Labs, Supplier No.:DLM-9TB-25, CAS No.: 666-52-4), ethyl acetate (VWR International, Supplier No.: BDH1123-19L, CAS No.: 141-78-6), dichloromethane (VWR International, Supplier No.: BDH1113-19L, CAS No.: 75-09-2), petroleum ether (VWR International, Supplier No.: BDH1145-19L, CAS No.: 64742-49-0), ammonium chloride (VWR 
International, Supplier No.: BDH9208-500G, CAS No.: 12125-02-9), sodium chloride (VWR International, Supplier No.: BDH9286-2.5KG, CAS No.: 7647-14-5), and silica gel 60 (Avantor, Supplier No.: V120-25, CAS No.: 63231-67-4). Quartz microscope slides (ChemGlass, Supplier No.: CGQ-0640-01) were used as substrates, and bonded samples were handled and stored using aluminum foil (Reynolds Wrap) to minimize contamination of surfaces. The mass and thickness of each quartz slide, and of each bonded assembly were measured using a balance (VWR 124B) and digital display micrometer (iGaging). Melt-bonding was performed on a lab hot plate (IKA C-MAG HS 10).

\section{SII. Synthesis of Photoresponsive Crystalline Adhesives}

\section{2-Nitrobenzyl Benzoate (1)}

A flame-dried $250-\mathrm{mL}$ round bottomed flask was wrapped in aluminum foil and charged with a solution of 2-nitrobenzyl alcohol $(15 \mathrm{mmol})$ in pyridine $(75 \mathrm{~mL})$. To the flask was added benzoyl chloride $(16.5 \mathrm{mmol})$ at $0{ }^{\circ} \mathrm{C}$ under a nitrogen atmosphere. The reaction mixture was kept at the same temperature for $3 \mathrm{~h}$, and the excess reagent was destroyed by adding water $(25 \mathrm{~mL})$. The reaction mixture was extracted with ethyl acetate, and the organic layer was washed with saturated aqueous ammonium chloride followed by brine, and then evaporated. Purification of the residue by column chromatography (silica gel 60) with petroleum ether/ethyl acetate (11:1) as an eluent yielded 1 as a white powder in $54 \%$ yield. ${ }^{1} \mathrm{H} \mathrm{NMR}\left(600 \mathrm{MHz}, \mathrm{CDCl}_{3}\right) \delta 8.1438$ (d, $J=8.4594 \mathrm{~Hz}, 1 \mathrm{H}), 8.103(\mathrm{~d}, J=7.1237 \mathrm{~Hz}, 2 \mathrm{H}), 7.7022-7.6414(\mathrm{~m}, 2 \mathrm{H}), 7.6044(\mathrm{t}, J=7.569 \mathrm{~Hz}$, 1H), 7.5331-7.4589 (m, 3H), 5.79119 (s, 2H). $\left.{ }^{13} \mathrm{C} \mathrm{NMR} \mathrm{(600} \mathrm{MHz,} \mathrm{CDCl}_{3}\right) \delta 165.9415,133.7947$, $133.4107,132.3931,129.7900,129.5892,128.9382,128.8041,128.5711,128.5711,125.1338$, 63.3542. 


\section{2-Nitrobenzyl 2-Naphthoate (2)}

A flame-dried $250-\mathrm{mL}$ round bottomed flask was wrapped in aluminum foil and charged with a solution of 2-nitrobenzyl alcohol $(15 \mathrm{mmol})$ in pyridine $(75 \mathrm{~mL})$. To the flask was added 2-naphthoyl chloride $(16.5 \mathrm{mmol})$ at $0{ }^{\circ} \mathrm{C}$ under a nitrogen atmosphere. The reaction mixture was kept at the same temperature for $3 \mathrm{~h}$, and the excess reagent was destroyed by adding water $(25 \mathrm{~mL})$. The reaction mixture was extracted with ethyl acetate, and the organic layer was washed with saturated aqueous ammonium chloride followed by brine, and then evaporated. Purification of the residue by column chromatography (silica gel 60) with petroleum ether/ethyl acetate $(11: 1)$ as an eluent yielded 2 as a white powder in $98 \%$ yield. ${ }^{1} \mathrm{H} \mathrm{NMR}\left(600 \mathrm{MHz}, \mathrm{CDCl}_{3}\right) \delta 8.946$ $(\mathrm{d}, J=8.251 \mathrm{~Hz}, 1 \mathrm{H}), 8.286(\mathrm{~d}, J=6.842 \mathrm{~Hz}, 1 \mathrm{H}), 8.166(\mathrm{~d}, J=8.210 \mathrm{~Hz}, 1 \mathrm{H}), 8.069(\mathrm{~d}, J=8.666 \mathrm{~Hz}$, 1H), $7.908(\mathrm{~d}, J=8.66 \mathrm{~Hz}, 1 \mathrm{H}), 7.739(\mathrm{~d}, J=7.298 \mathrm{~Hz}, 1 \mathrm{H}), 7.666(\mathrm{t}, J=7.298 \mathrm{~Hz}, 1 \mathrm{H}), 7.631(\mathrm{t}$, $J=8.210 \mathrm{~Hz}, 1 \mathrm{H}), 7.573-7.500(\mathrm{~m}, 3 \mathrm{H}), 5.874(\mathrm{~s}, 2 \mathrm{H}) .{ }^{13} \mathrm{C} \mathrm{NMR}\left(600 \mathrm{MHz}, \mathrm{CDCl}_{3}\right) \delta 166.6654$, 133.9451, 133.9012, 133.8400, 132.4341, 131.5119, 130.5181, 129.1657, 128.8463, 128.6366, $128.0661,126.3907,126.2610,125.7077,125.1902,124.5409,63.4743$.

\section{2-Nitrobenzyl 1-Naphthoate (3)}

A flame-dried $250-\mathrm{mL}$ round bottomed flask was wrapped in aluminum foil and charged with a solution of 2-nitrobenzyl alcohol $(15 \mathrm{mmol})$ in pyridine $(75 \mathrm{~mL})$. To the flask was added 1-naphthoyl chloride $(16.5 \mathrm{mmol})$ at $0{ }^{\circ} \mathrm{C}$ under a nitrogen atmosphere. The reaction mixture was kept at the same temperature for $3 \mathrm{~h}$, and the excess reagent was destroyed by adding water $(25 \mathrm{~mL})$. The reaction mixture was extracted with ethyl acetate, and the organic layer was washed with saturated aqueous ammonium chloride followed by brine, and then evaporated. Purification of the residue by column chromatography (silica gel 60) with petroleum ether/ethyl acetate 
(11:1) as an eluent yielded 3 as a white powder in $89 \%$ yield. ${ }^{1} \mathrm{H} N M R\left(600 \mathrm{MHz}, \mathrm{CDCl}_{3}\right) \delta 8.676$ (s, 1H), $8.162(\mathrm{~d}, J=7.744 \mathrm{~Hz}, 1 \mathrm{H}), 8.106(\mathrm{~d}, J=8.340 \mathrm{~Hz}, 1 \mathrm{H}), 7.983(\mathrm{~d}, J=7.744 \mathrm{~Hz}, 1 \mathrm{H}), 7.911$ $(t, J=8.638 \mathrm{~Hz}, 2 \mathrm{H}), 7.738(\mathrm{~d}, J=7.744 \mathrm{~Hz}, 1 \mathrm{H}), 7.678(\mathrm{t}, J=7.744 \mathrm{~Hz}, 1 \mathrm{H}), 7.619(\mathrm{t}, J=7.446 \mathrm{~Hz}$, 1H), $7.567(t, J=6.553 \mathrm{~Hz}, 1 \mathrm{H}), 7.522(\mathrm{t}, J=7.446 \mathrm{~Hz}, 1 \mathrm{H}), 5.854(\mathrm{~s}, 2 \mathrm{H}) .{ }^{13} \mathrm{C} \mathrm{NMR}\left(600 \mathrm{MHz}, \mathrm{CDCl}_{3}\right)$ $\delta 135.7447,133.8324,131.4622,129.4610,128.9779,128.8277,128.5445,128.4115,127.8292$, $126.8259,125.1918,125.1641,63.4730$.

\section{2-Nitrobenzyl 1-Adamantane Carboxylate (4)}

A flame-dried $250-\mathrm{mL}$ round bottomed flask was wrapped in aluminum foil and charged with a solution of 2-nitrobenzyl alcohol $(15 \mathrm{mmol})$ in pyridine $(75 \mathrm{~mL})$. To the flask was added 1-adamantanecarbonyl chloride $(16.5 \mathrm{mmol})$ at $0{ }^{\circ} \mathrm{C}$ under a nitrogen atmosphere. The reaction mixture was kept at the same temperature for $3 \mathrm{~h}$, and the excess reagent was destroyed by adding water $(25 \mathrm{~mL})$. The reaction mixture was extracted with ethyl acetate, and the organic layer was washed with saturated aqueous ammonium chloride followed by brine, and then evaporated. Purification of the residue by column chromatography (silica gel 60) with petroleum ether/ethyl acetate (11:1) as an eluent yielded 4 as a white powder in $76 \%$ yield. ${ }^{1} \mathrm{H}$ NMR $\left.\left(600 \mathrm{MHz}^{\mathrm{CDCl}}\right)_{3}\right) \delta 8.113(\mathrm{~d}, J=8.045 \mathrm{~Hz}, 1 \mathrm{H}), 7.673(\mathrm{t}, J=8.045 \mathrm{~Hz}, 1 \mathrm{H}), 7.560(\mathrm{~d}, J=8.045 \mathrm{~Hz}$, 1H), $7.510(t, J=8.045 \mathrm{~Hz}, 1 \mathrm{H}), 5.509(\mathrm{~s}, 2 \mathrm{H}), 2.066(\mathrm{~m}, 4 \mathrm{H}), 1.970(\mathrm{~m}, 2 \mathrm{H}), 1.756(\mathrm{~m}, 3 \mathrm{H}), 1.564$ (m, 6H). ${ }^{13} \mathrm{C} \mathrm{NMR}\left(600 \mathrm{MHz}, \mathrm{CDCl}_{3}\right) \delta 133.6135,128.8611,128.6306,125.0264,62.7258,38.8371$, $38.2797,36.4446,36.3171,27.8987,27.6792$.

\section{SIII. NMR Spectroscopy}

NMR spectroscopy was performed on a Bruker $600 \mathrm{MHz}$ Avance III HD spectrometer outfitted with a $5 \mathrm{~mm} \mathrm{BB}(\mathrm{F}) \mathrm{O}$ probe and controlled by Topspin 3.2 pl6. Quartz NMR tubes 
(ChemGlass, Supplier No.: C-S-5-600-QTZ-8) were used throughout this report. NMR spectra of interest are presented in Figures S1-S16.

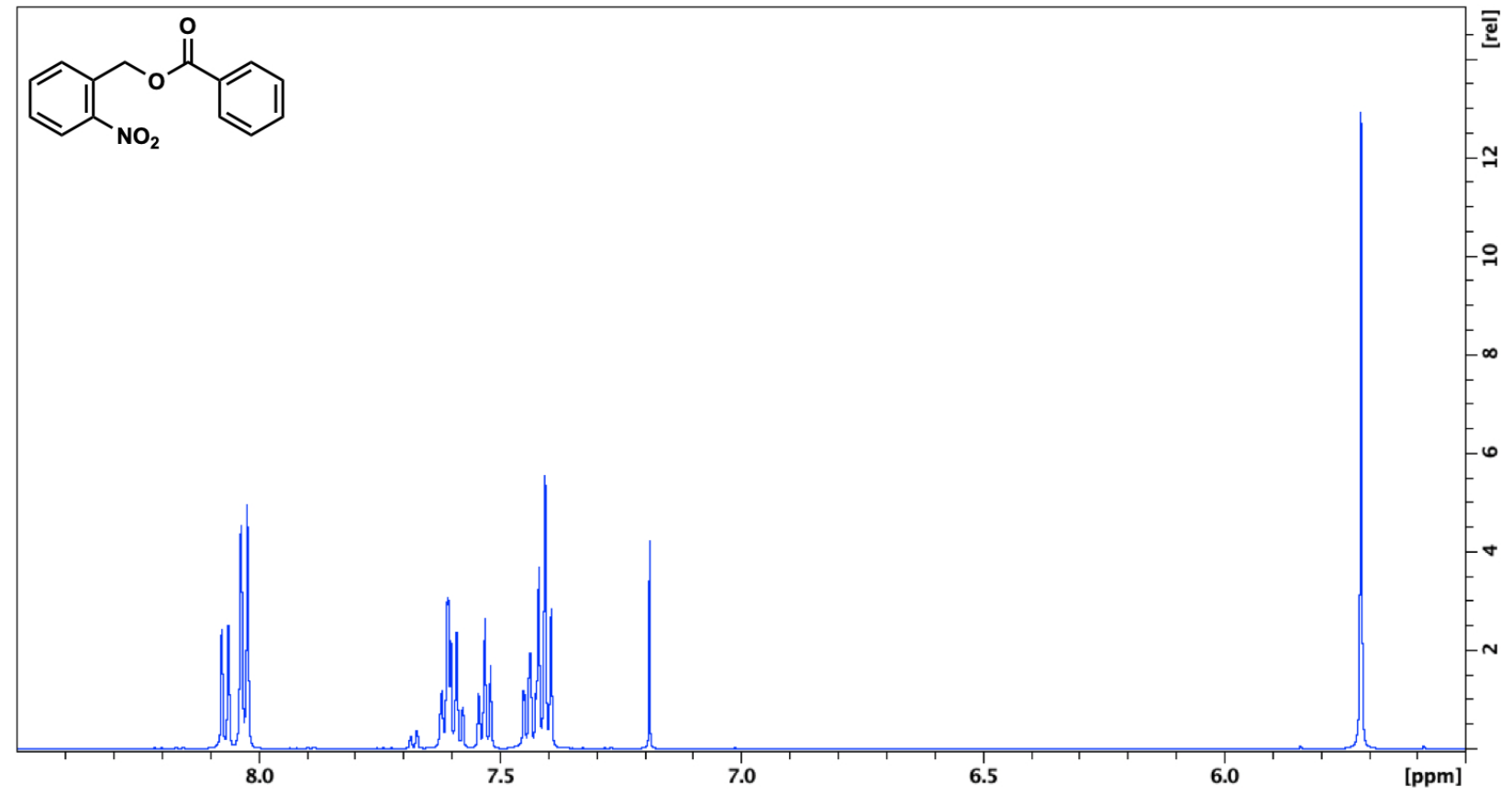

Figure S1. ${ }^{1} \mathrm{H}$ NMR spectrum of 2-nitrobenzyl benzoate (1).

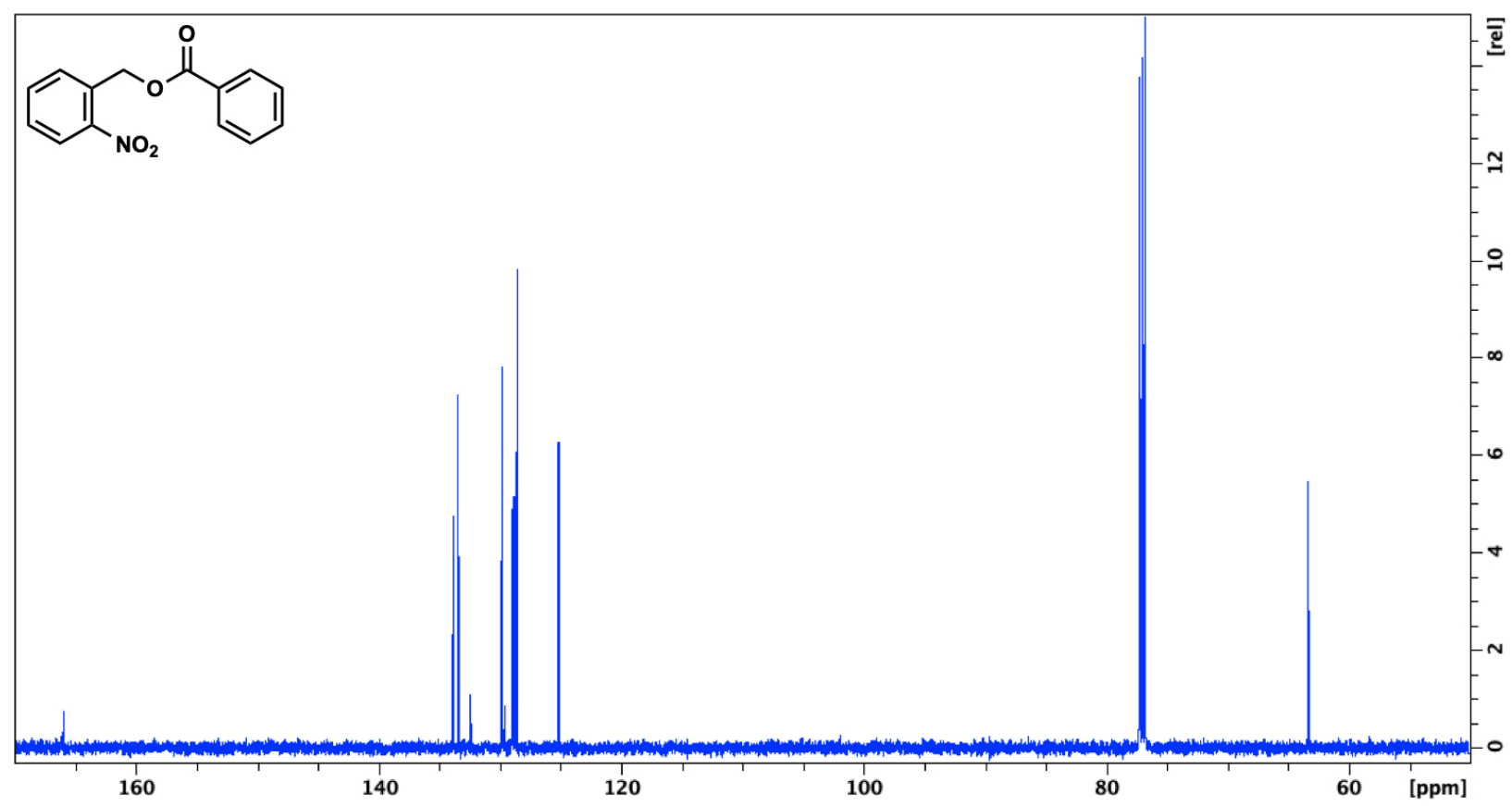

Figure S2. ${ }^{13} \mathrm{C}$ NMR spectrum of 2-nitrobenzyl benzoate (1). 


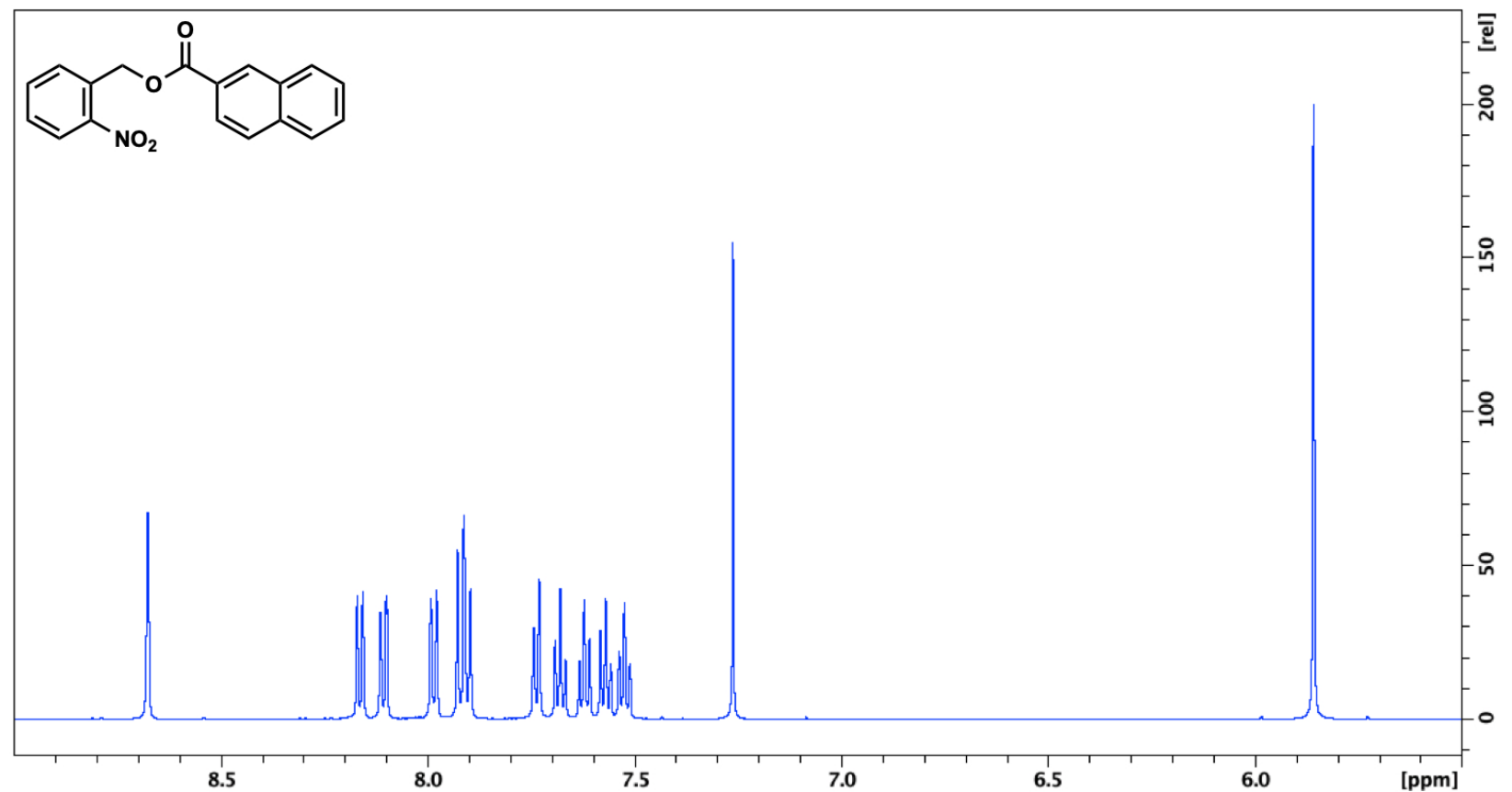

Figure S3. ${ }^{1} \mathrm{H}$ NMR spectrum of 2-nitrobenzyl 2-naphthoate (2).

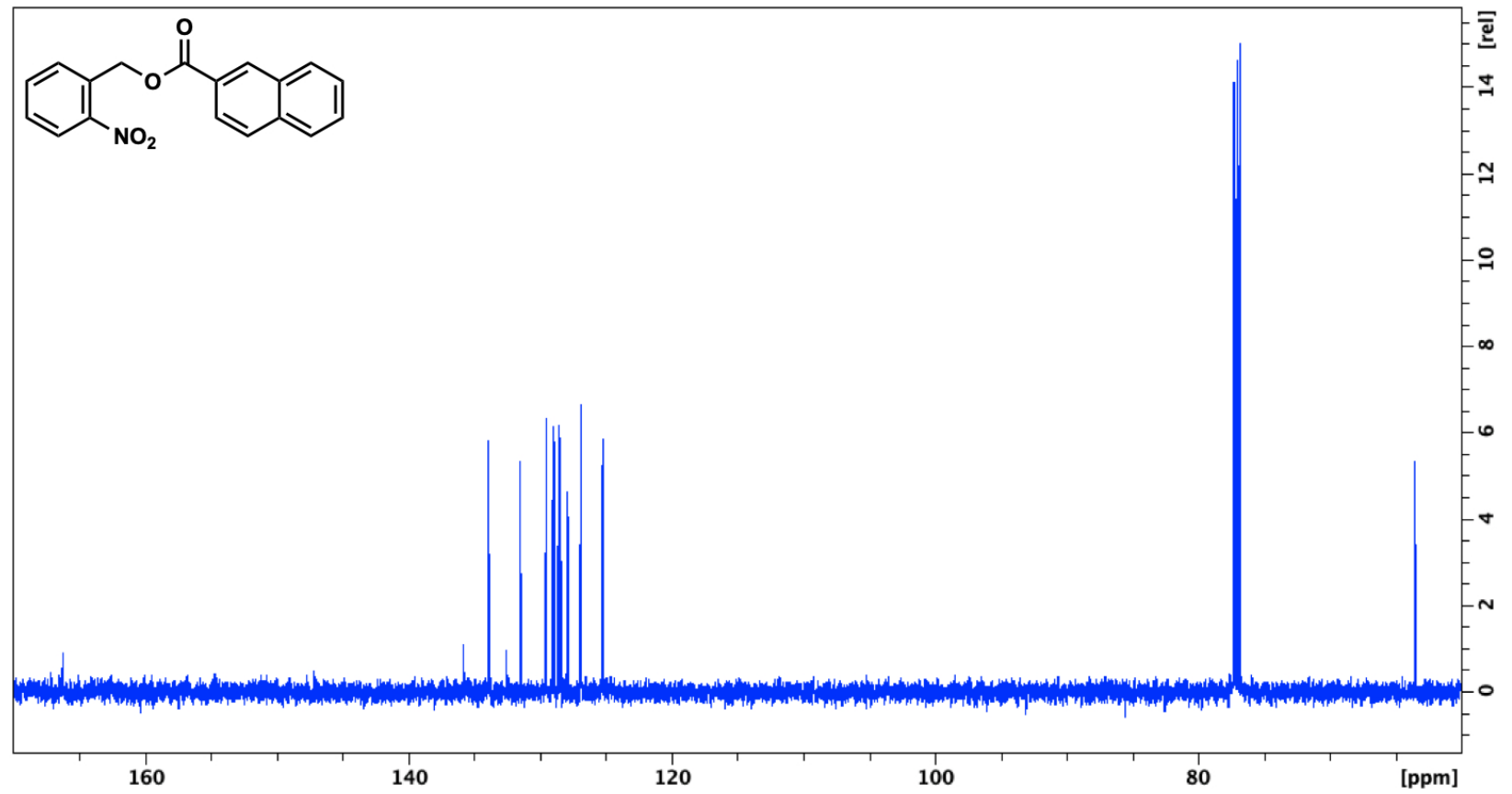

Figure S4. ${ }^{13} \mathrm{C}$ NMR spectrum of 2-nitrobenzyl 2-naphthoate (2). 


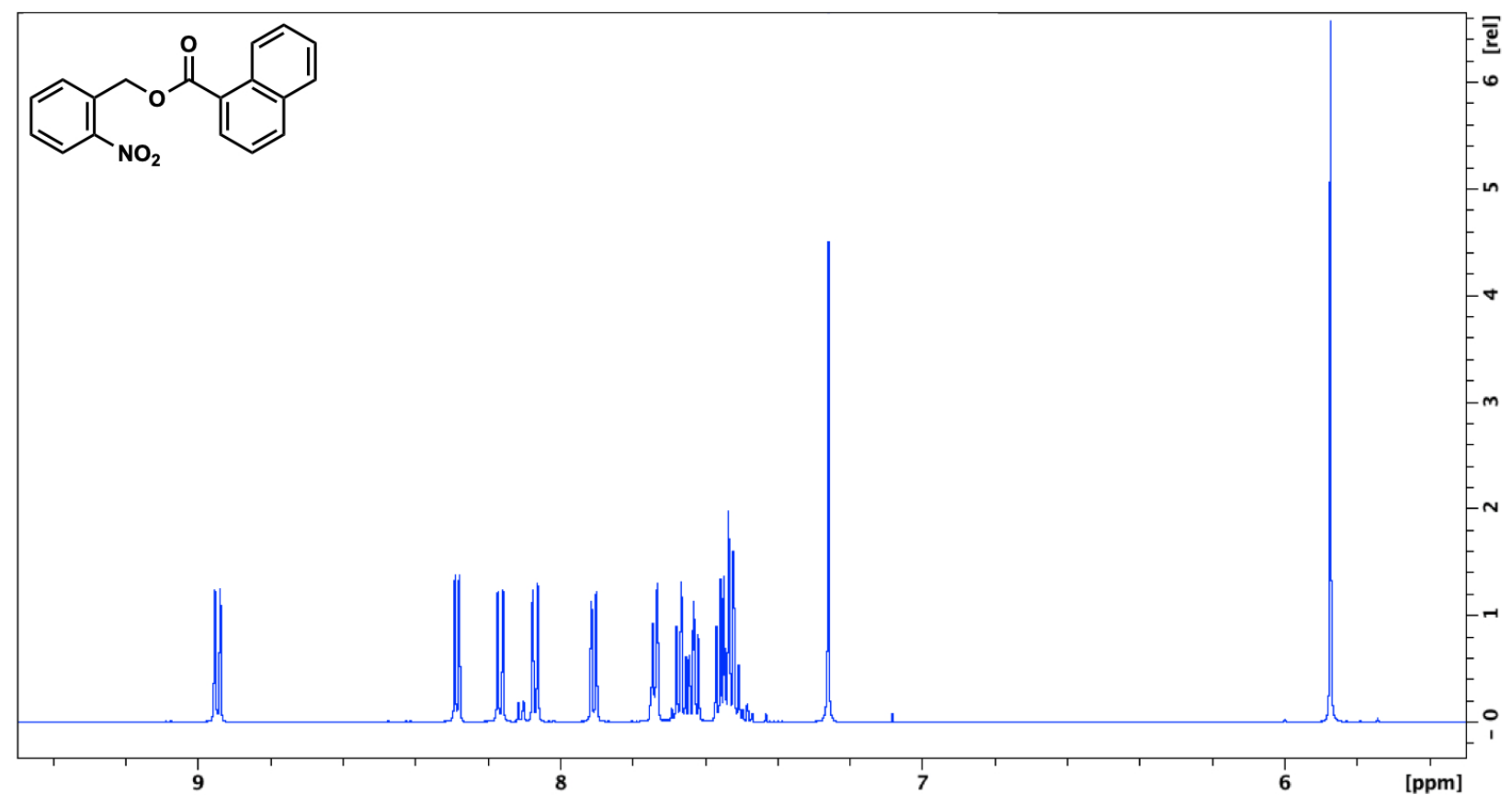

Figure S5. ${ }^{1} \mathrm{H}$ NMR spectrum of 2-nitrobenzyl 1-naphthoate (3).

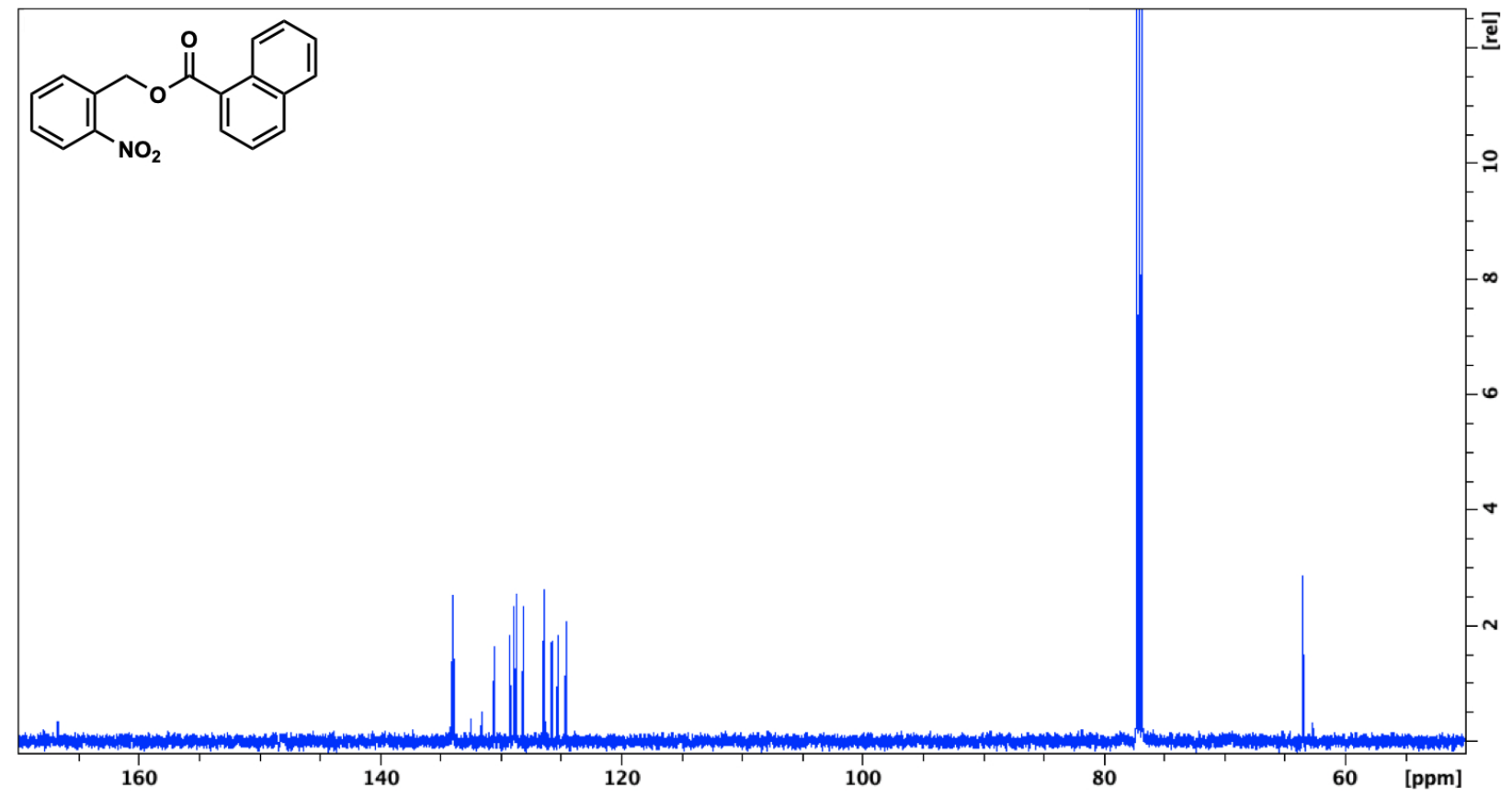

Figure S6. ${ }^{13} \mathrm{C}$ NMR spectrum of 2-nitrobenzyl 1-naphthoate (3). 


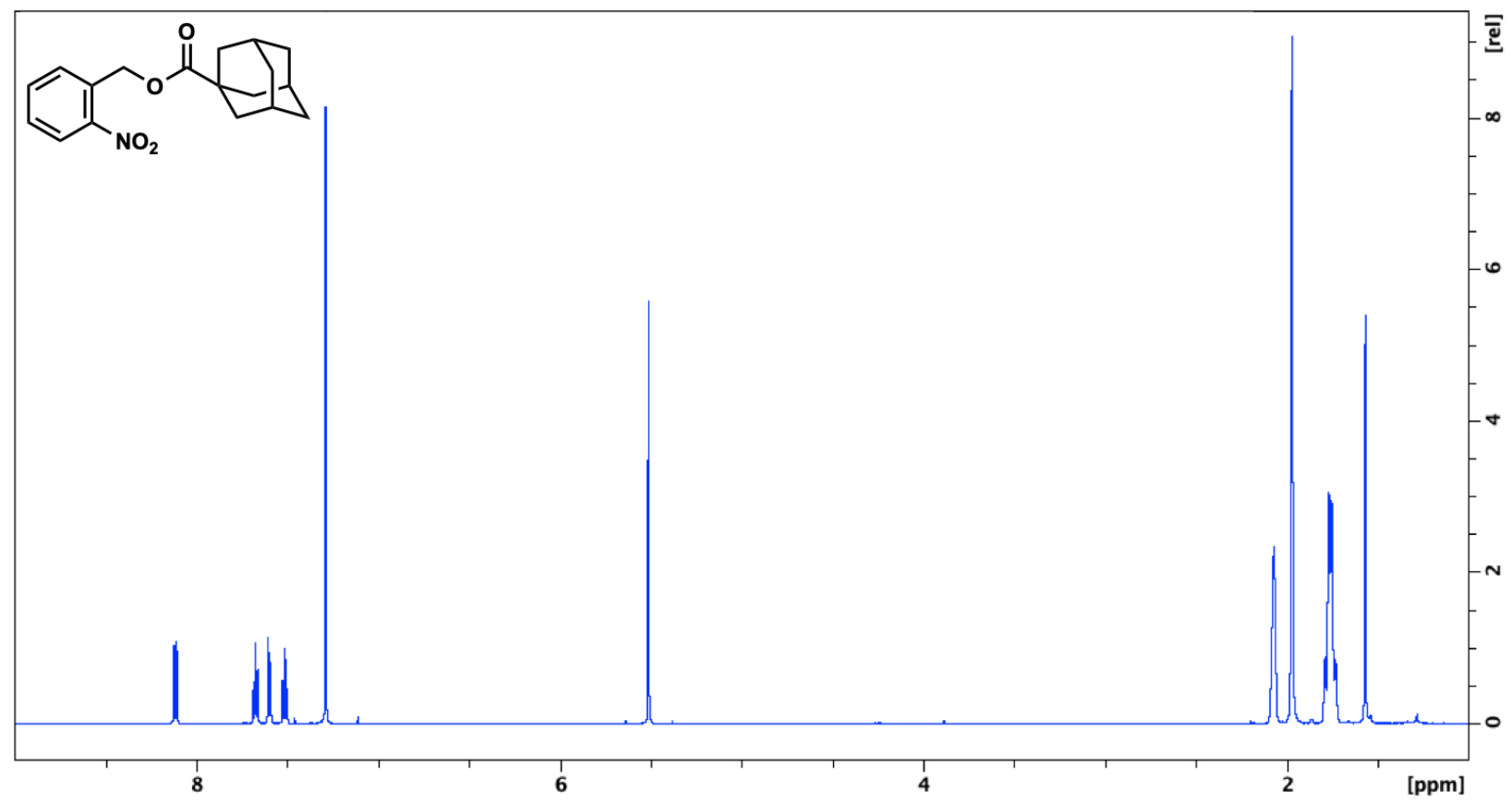

Figure S7. ${ }^{1} \mathrm{H}$ NMR spectrum of 2-nitrobenzyl 1-adamantane carboxylate (4).

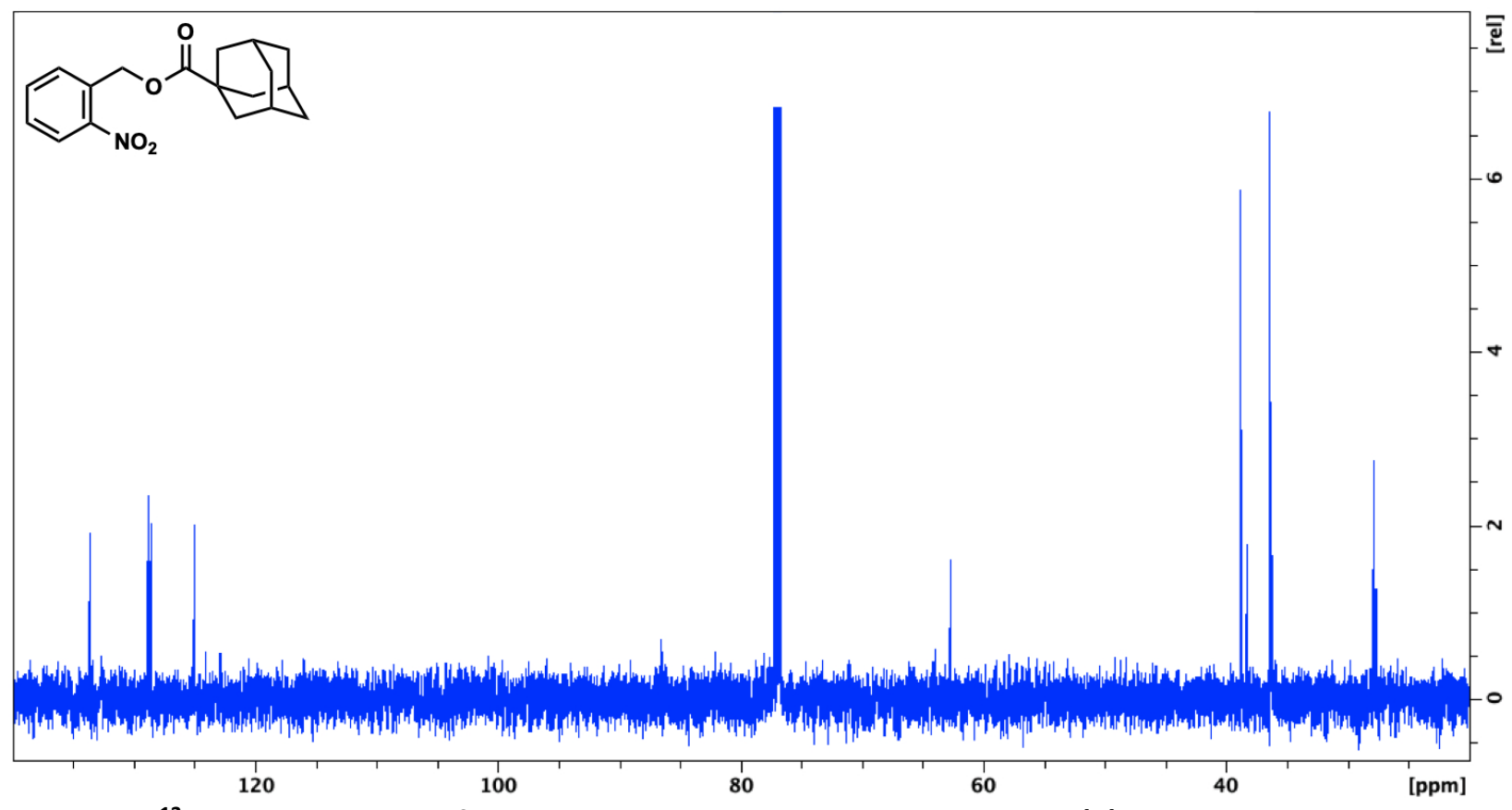

Figure S8. ${ }^{13} \mathrm{C}$ NMR spectrum of 2-nitrobenzyl 1-adamantane carboxylate (4).

Page 9 of 52 


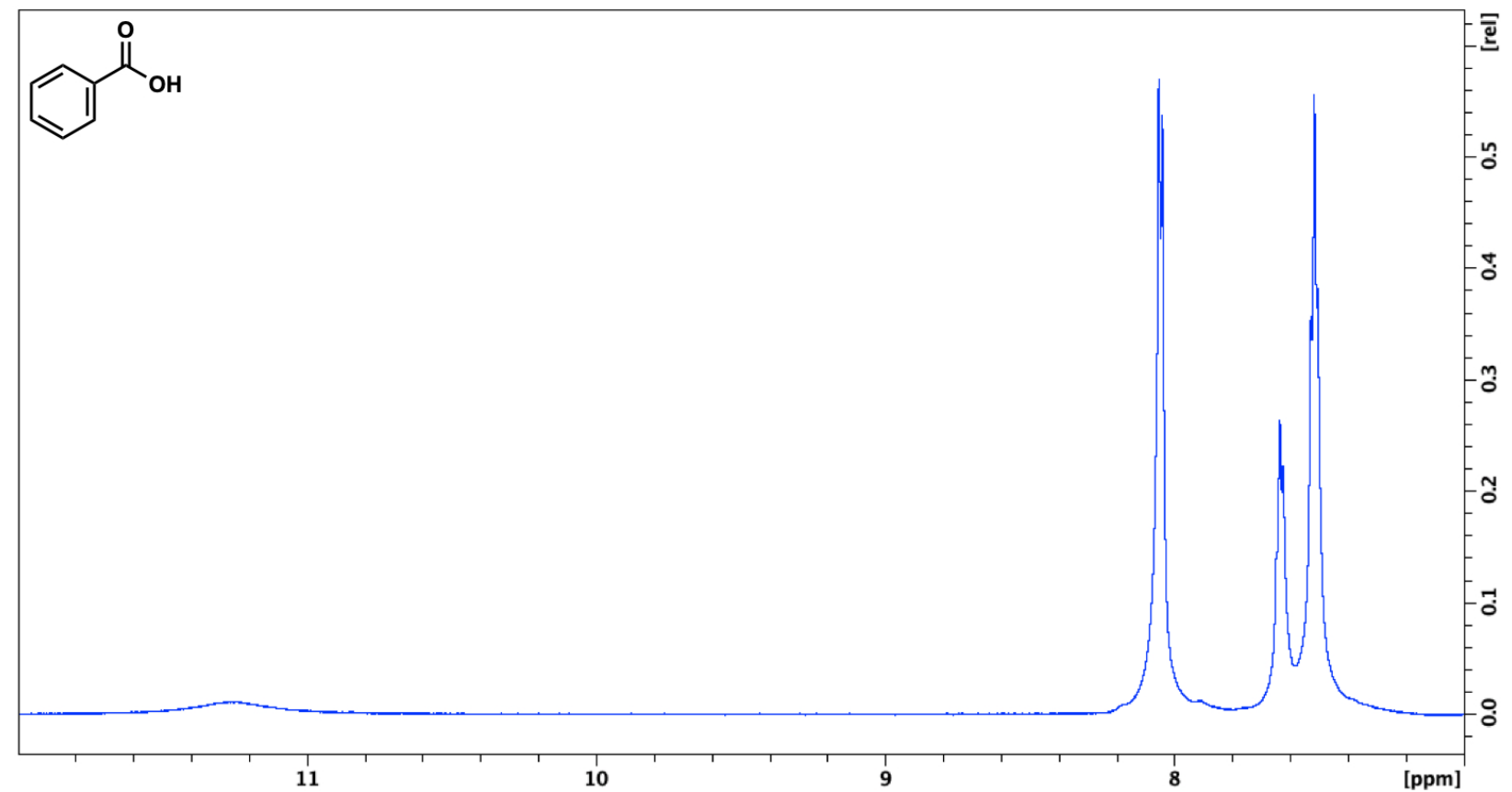

Figure S9. ${ }^{1} \mathrm{H}$ NMR spectrum of benzoic acid.

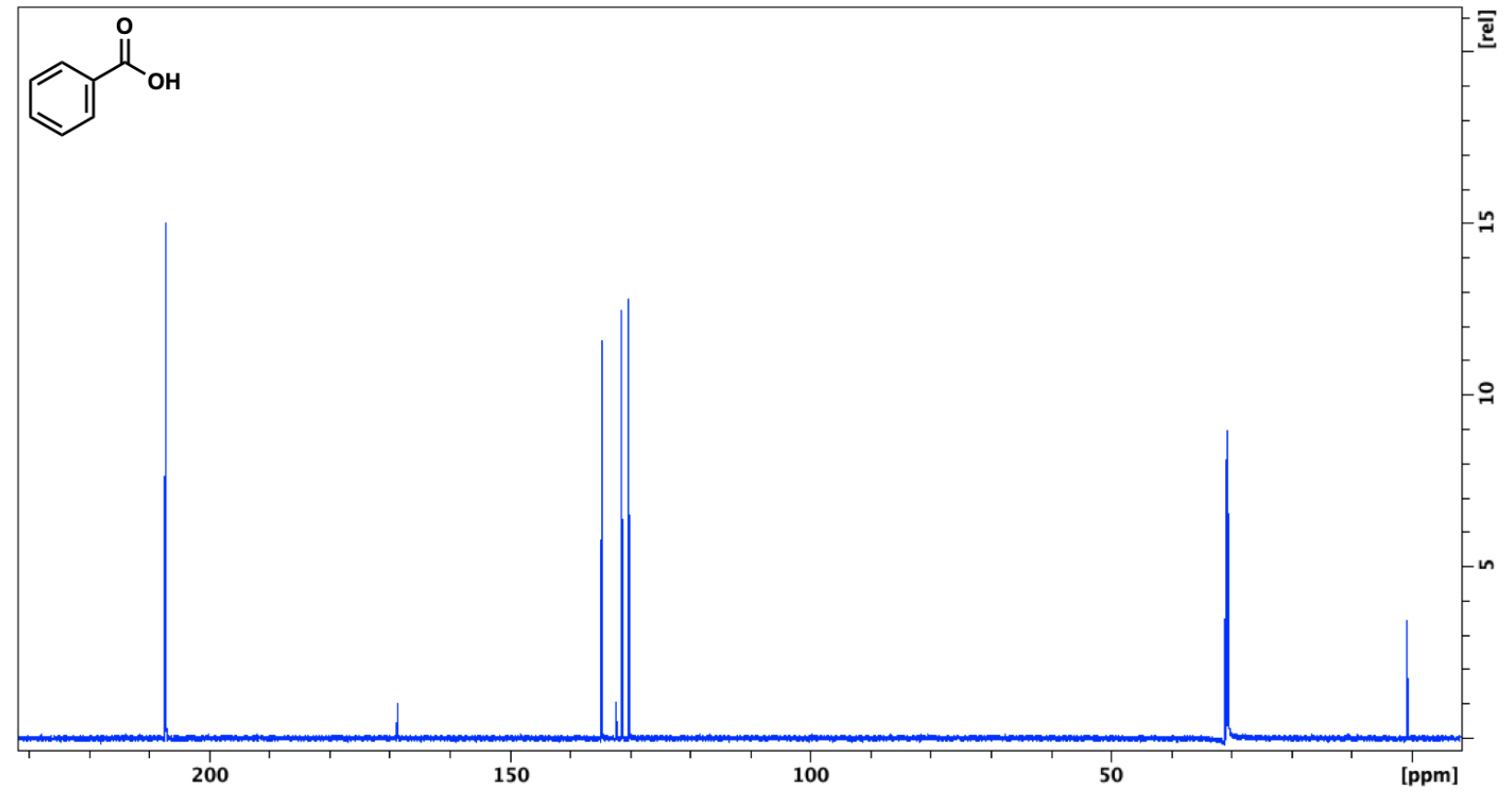

Figure S10. ${ }^{13} \mathrm{C}$ NMR spectrum of benzoic acid. 


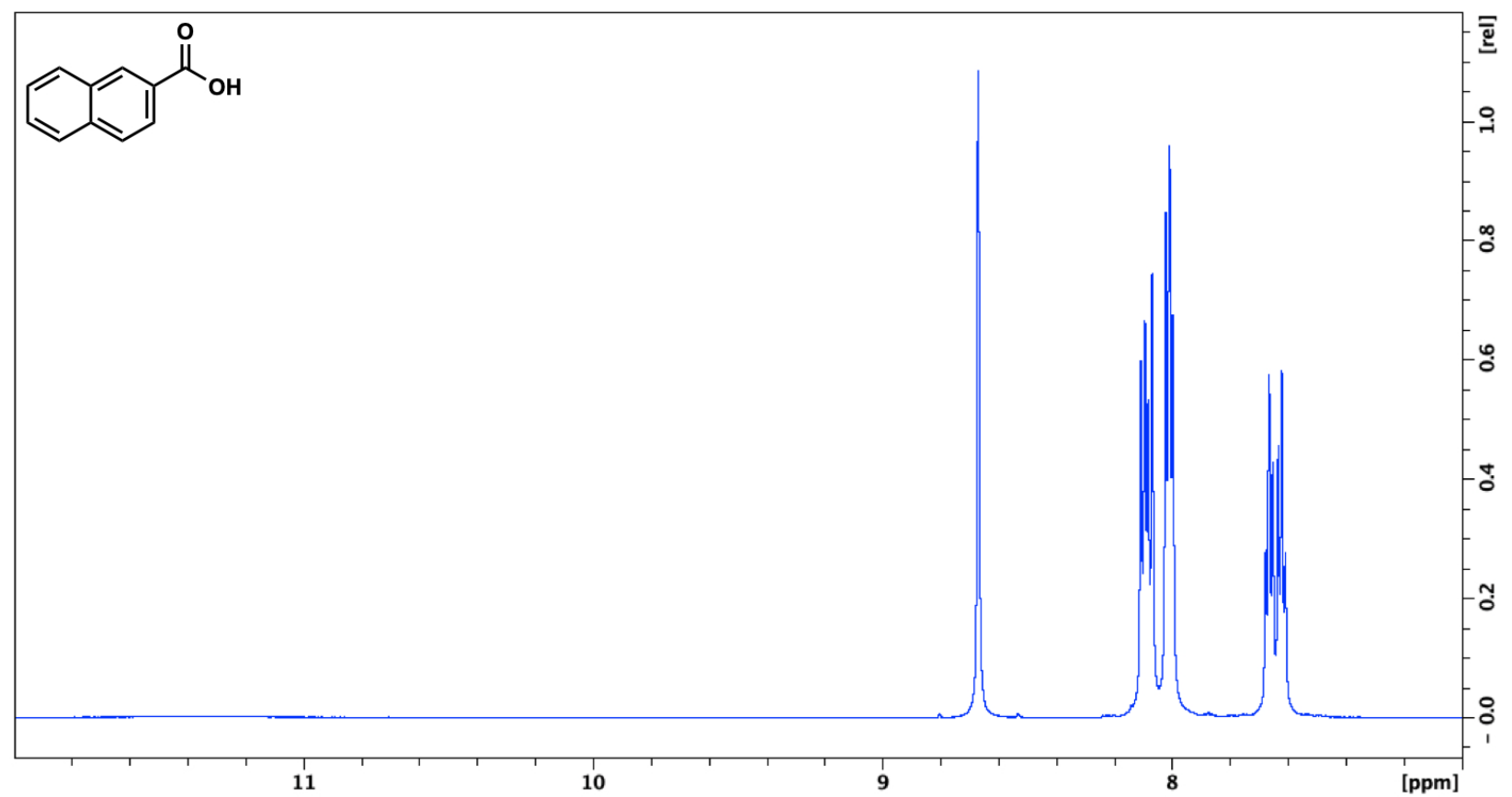

Figure S11. ${ }^{1} \mathrm{H}$ NMR spectrum of 2-naphthoic acid.

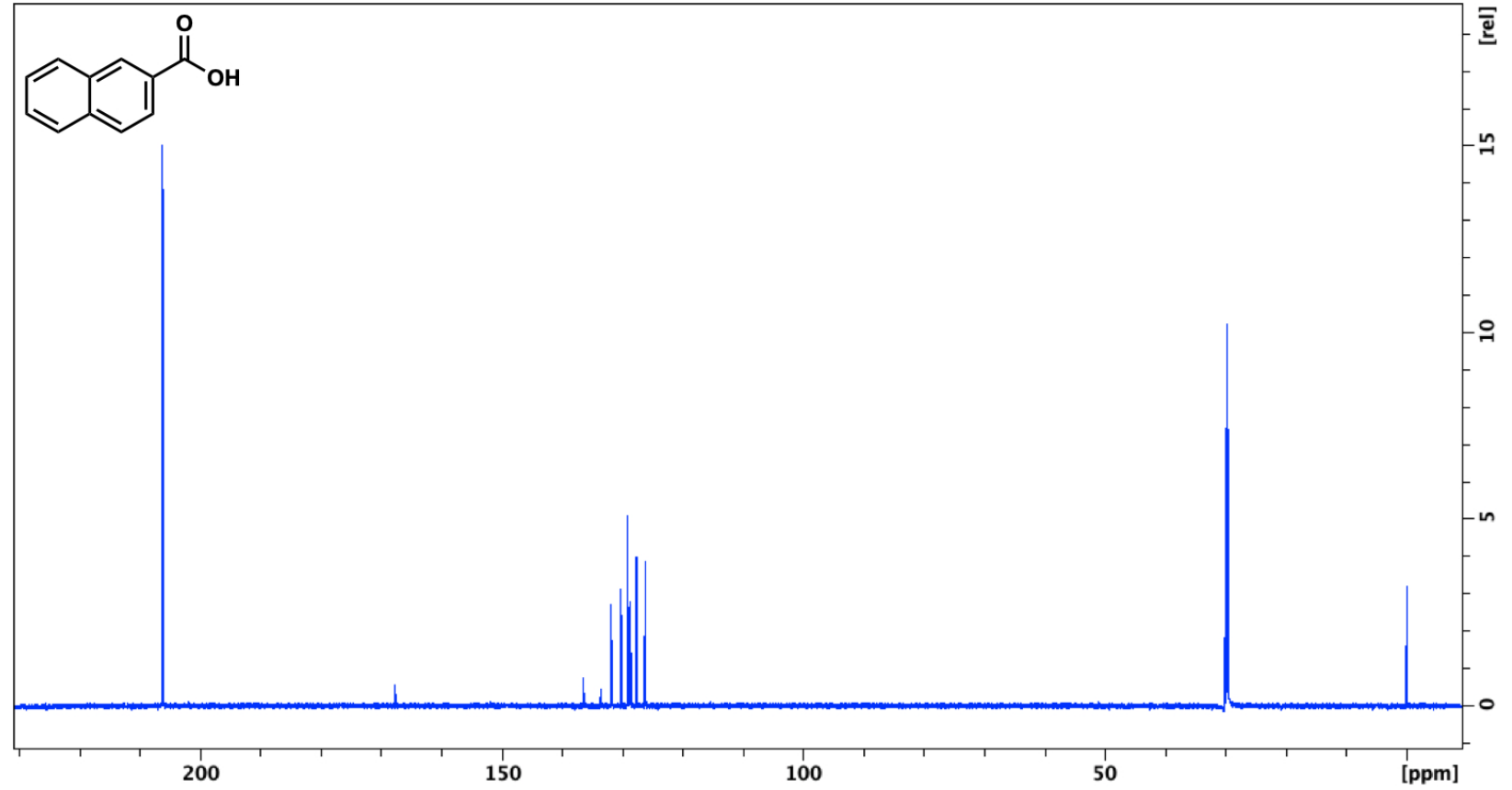

Figure S12. ${ }^{13} \mathrm{C}$ NMR spectrum of 2-naphthoic acid. 


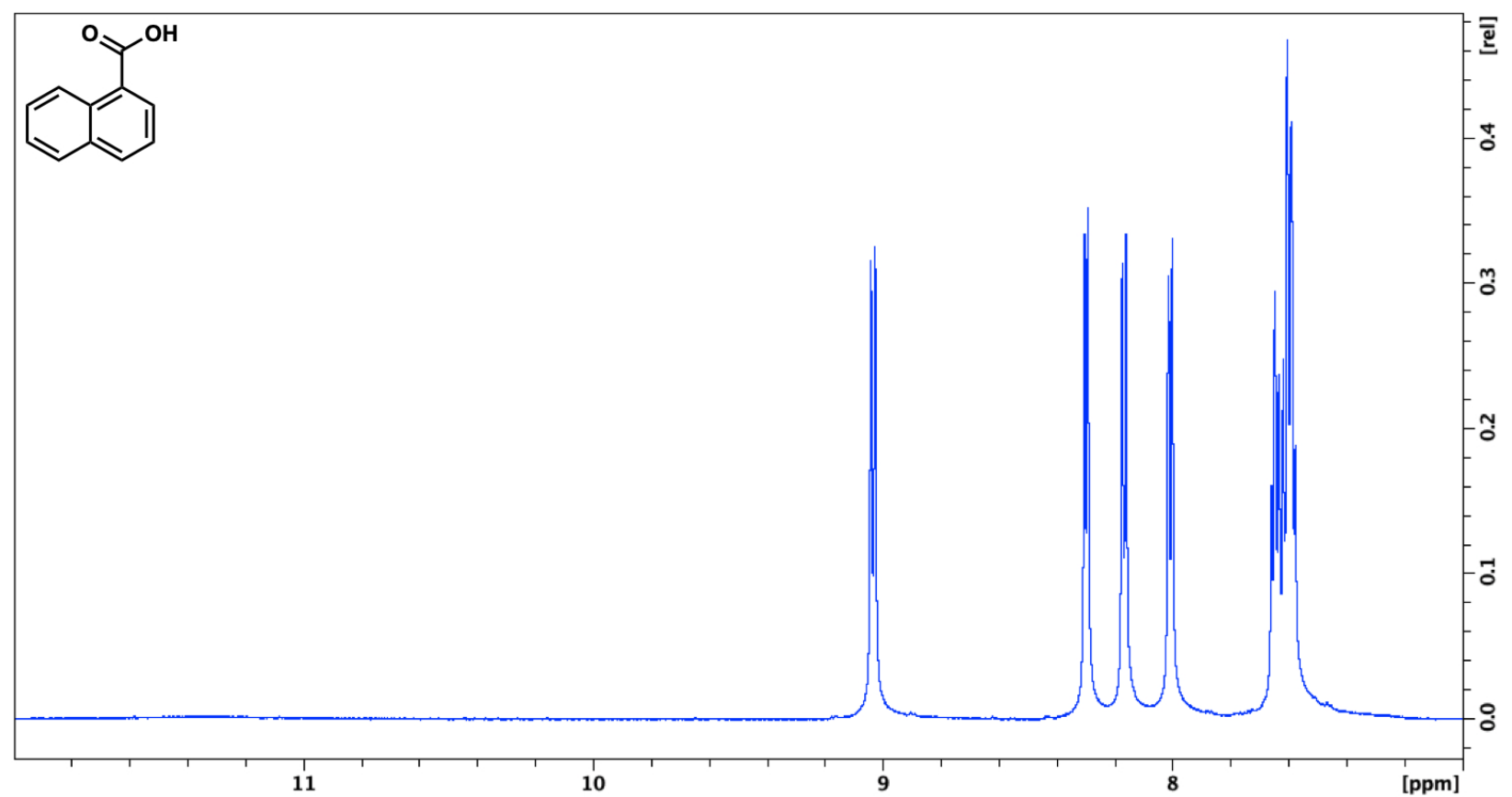

Figure S13. ${ }^{1} \mathrm{H}$ NMR spectrum of 1-naphthoic acid.

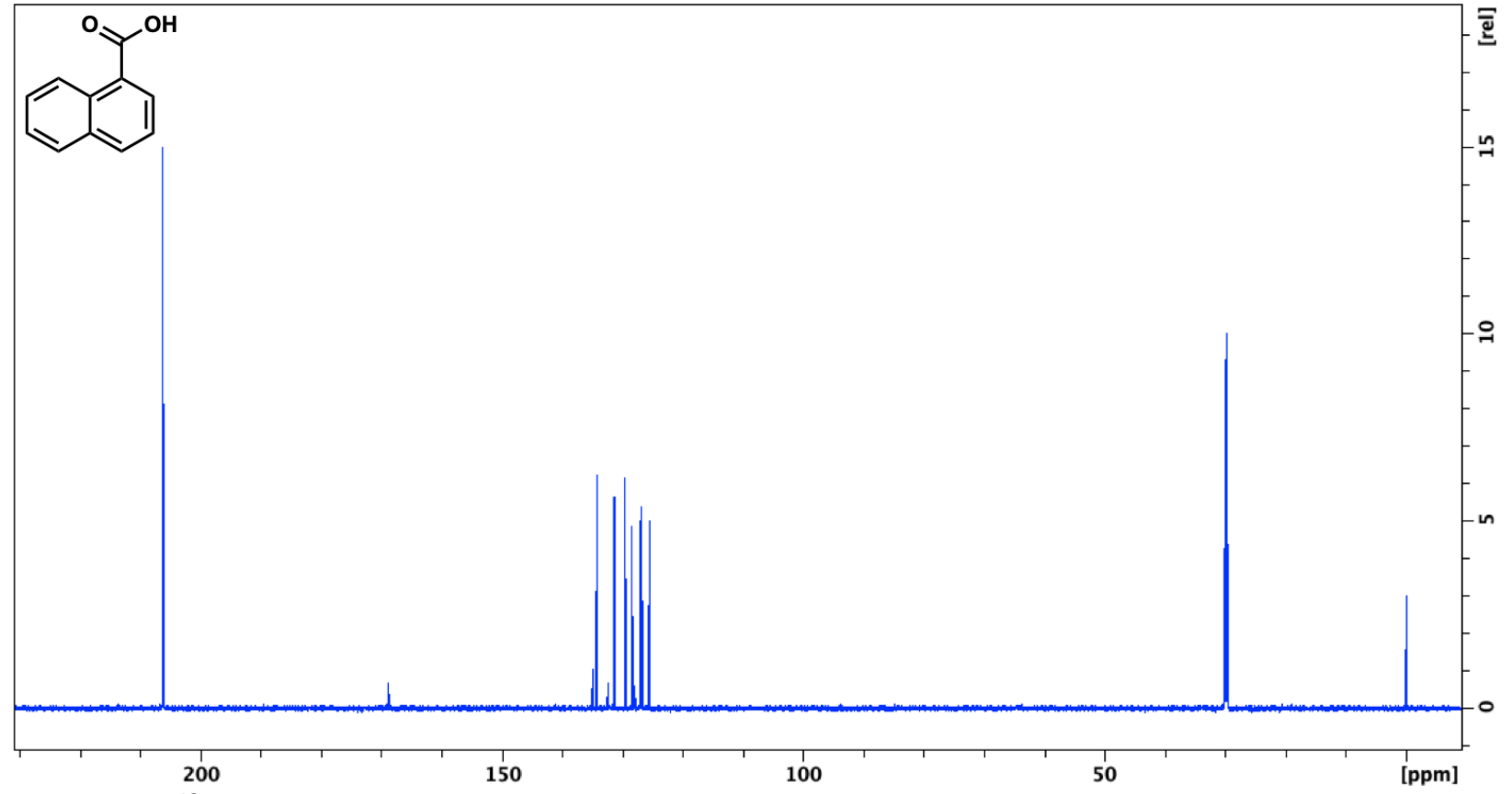

Figure S14. ${ }^{13} \mathrm{C}$ NMR spectrum of 1-naphthoic acid. 


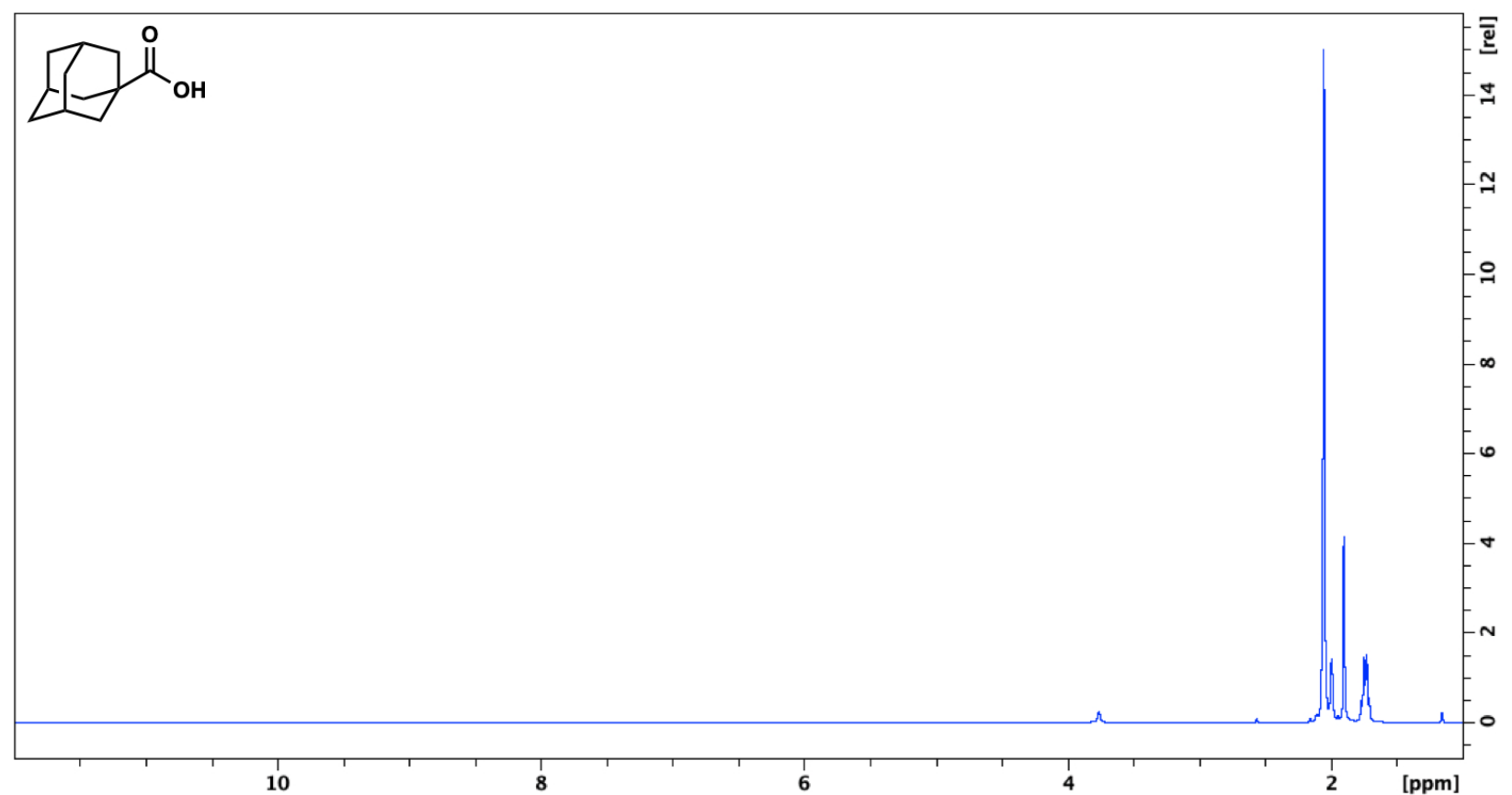

Figure S15. ${ }^{1} \mathrm{H}$ NMR spectrum of adamantane-1-carboxylic acid.

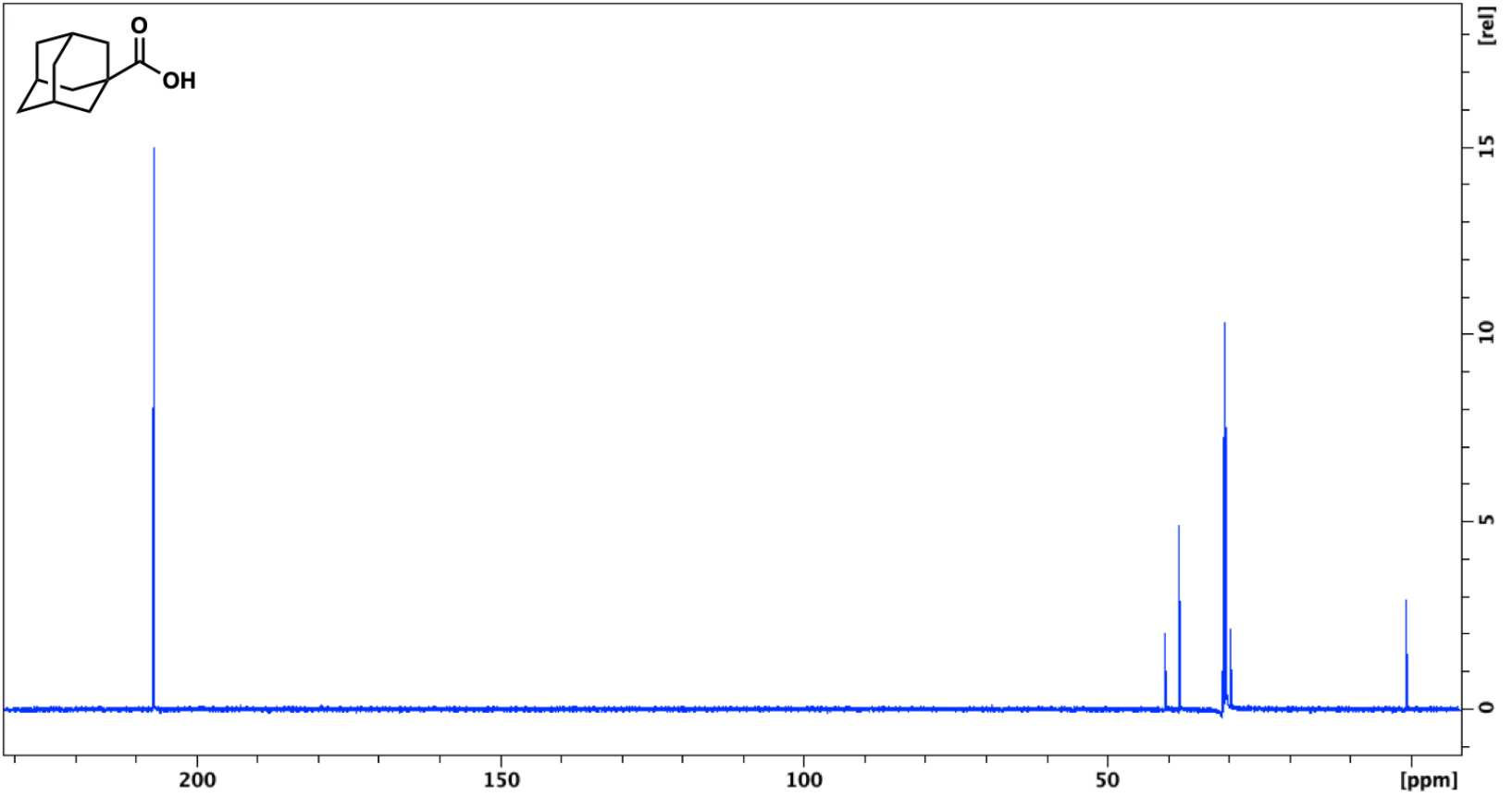

Figure S16. ${ }^{13} \mathrm{C}$ NMR spectrum of adamantane-1-carboxylic acid. 


\section{SIV. Growing Single Crystals of Photoresponsive Crystalline Adhesives}

\section{2-Nitrobenzyl Benzoate (1)}

A $100 \mathrm{mM}$ stock solution was prepared by dissolving 1 in petroleum ether and filtering the resulting solution through a GHP Acrodisc ${ }^{\circledR} 25-\mathrm{mm}$ syringe filter with a $0.2 \mathrm{~mm}$ GHP membrane (Pall Industries, Supplier No.: MS-3201). 1-mL aliquots were deposited into borosilicate glass vials (ChemGlass, Supplier No.: CG-4916-30). Each vial was capped and punctured with an 18-gauge hypodermic needle (Air-Tite, Supplier No.: N181). Crystallization was allowed to proceed via slow evaporation of solvent, yielding single crystals suitable for structure determination.

\section{2-Nitrobenzyl 2-Naphthoate (2)}

A 100 mM stock solution was prepared by dissolving 2 in petroleum ether and filtering the resulting solution through a GHP Acrodisc ${ }^{\circledast} 25-\mathrm{mm}$ syringe filter with a $0.2 \mathrm{~mm}$ GHP membrane. 1-mL aliquots were deposited into borosilicate glass vials. Each vial was capped and punctured with an 18-gauge hypodermic needle. Crystallization was allowed to proceed via slow evaporation of solvent, yielding single crystals suitable for structure determination.

\section{2-Nitrobenzyl 1-Naphthoate (3)}

A $100 \mathrm{mM}$ stock solution was prepared by dissolving 3 in petroleum ether and filtering the resulting solution through a GHP Acrodisc ${ }^{\oplus} 25-\mathrm{mm}$ syringe filter with a $0.2 \mathrm{~mm}$ GHP membrane. 1-mL aliquots were deposited into borosilicate glass vials. Each vial was capped and punctured with an 18-gauge hypodermic needle. Crystallization was allowed to proceed via slow evaporation of solvent, yielding single crystals suitable for structure determination. 


\section{2-Nitrobenzyl 1-Adamantane Carboxylate (4)}

A $1 \mathrm{mM}$ solution was prepared by dissolving 4 in 9:1 dichloromethane: petroleum ether and filtering the resulting solution through a GHP Acrodisc ${ }^{\circledR} 25-\mathrm{mm}$ syringe filter with a $0.2 \mathrm{~mm}$ GHP. 1-mL aliquots were deposited into borosilicate glass vials. Each vial was capped and punctured with an 18-gauge hypodermic needle. Crystallization was allowed to proceed via slow evaporation of solvent, yielding single crystals suitable for structure determination.

\section{1,2-Bis(2-nitrophenyl)ethane (5)}

A $100 \mathrm{mM}$ stock solution was prepared by dissolving the impure product from the attempted synthesis of $\mathbf{2}$ in petroleum ether and filtering the resulting solution through a GHP Acrodisc 25 -mm syringe filter with a $0.2 \mathrm{~mm}$ GHP membrane. 1-mL aliquots were deposited into borosilicate glass vials. Each vial was capped and punctured with an 18-gauge hypodermic needle. Crystallization was allowed to proceed via slow evaporation of solvent, yielding multiple single crystals suitable for structure determination. When the crystals were analyzed, they were found to contain 1,2-bis(2-nitrophenyl)ethane. The structure of 5 has previously been reported. ${ }^{1}$ $\underline{\text { 2-Nitrobenzyl alcohol }}$

Monoclinic polymorph (6): A 100 mM stock solution was prepared by dissolving 2nitrobenzyl alcohol in petroleum ether and filtering the resulting solution through a GHP Acrodisc $^{\oplus} 25$-mm syringe filter with a $0.2 \mathrm{~mm}$ GHP membrane. 1-mL aliquots were deposited into borosilicate glass vials. Each vial was capped and punctured with an 18-gauge hypodermic needle. Crystallization was allowed to proceed via slow evaporation of solvent, yielding multiple single crystals suitable for structure determination. 
Triclinic polymorph (7): In the same experiment as described immediately above, a 100 $\mathrm{mM}$ stock solution was prepared by dissolving 2-nitrobenzyl alcohol in petroleum ether and filtering the resulting solution through a GHP Acrodisc ${ }^{\circledR} 25-\mathrm{mm}$ syringe filter with a $0.2 \mathrm{~mm}$ GHP membrane. 1-mL aliquots were deposited into borosilicate glass vials. Each vial was capped and punctured with an 18-gauge hypodermic needle. Crystallization was allowed to proceed via slow evaporation of solvent, yielding multiple single crystals suitable for structure determination. Single crystals of both the monoclinic and triclinic crystal structure crystallized under the same experimental conditions as controlled for.

\section{SV. Crystal Structure Determination via Single Crystal X-Ray Diffraction}

Crystals suitable for diffraction were shipped to Dr. Richard Staples at the University of Michigan Center for Crystallographic Research. There the crystals were mounted on a nylon loop with paratone oil on a Bruker APEX-II CCD diffractometer. The crystals were kept at $173 \pm 2 \mathrm{~K}$ during data collection. Using Olex $2,{ }^{2}$ the structures were solved with the ShelXT structure solution program, ${ }^{3}$ using the Intrinsic Phasing solution method. Each model was refined with version XL using Lease Squares minimization. ${ }^{4}$ The crystal structures of benzoic acid, 2-naphthoic acid, 1-naphthoic acid, and adamantane-1-carboxylic acid are available on the Cambridge Structural Database (CSD-REFCODE: BENZAC01, NAPHAC02, NAPOAC01, and VIDSIK, respectively). ${ }^{5}$ Visualizations of the unit cell or relevant compounds are provided in Figures S17-

\section{S31.}


Table S1. Crystallographic data of 1-4.

\begin{tabular}{|c|c|c|c|c|c|c|c|}
\hline & 1 & 2 & 3 & 4 & 5 & 6 & 7 \\
\hline formula & $\mathrm{C}_{14} \mathrm{H}_{11} \mathrm{NO}_{4}$ & $\mathrm{C}_{18} \mathrm{H}_{13} \mathrm{NO}_{4}$ & $\mathrm{C}_{18} \mathrm{H}_{13} \mathrm{NO}_{4}$ & $\mathrm{C}_{18} \mathrm{H}_{21} \mathrm{NO}_{4}$ & $\mathrm{C}_{14} \mathrm{H}_{10} \mathrm{~N}_{2} \mathrm{O}_{4}$ & $\mathrm{C}_{7} \mathrm{H}_{7} \mathrm{NO}_{3}$ & $\mathrm{C}_{10.5} \mathrm{H}_{10.5} \mathrm{~N}_{1.5} \mathrm{O}_{4.5}$ \\
\hline$D_{\text {calc. }}\left(\mathrm{g} \mathrm{cm}^{-3}\right)$ & 1.448 & 1.429 & 1.444 & 1.376 & 1.482 & 1.475 & 1.467 \\
\hline$\mu\left(\mathrm{mm}^{-1}\right)$ & 0.900 & 0.102 & 0.103 & 0.794 & 0.111 & 0.997 & 0.992 \\
\hline $\begin{array}{l}\text { formula } \\
\text { weight }\end{array}$ & 257.24 & 307.29 & 307.29 & 315.36 & 270.24 & 153.14 & 229.70 \\
\hline color & colorless & colorless & colorless & colorless & Yellow & colorless & colorless \\
\hline shape & plate & needle & needle & needle & needle & needle & needle \\
\hline size $\left(\mathrm{mm}^{3}\right)$ & $\begin{array}{c}0.28 \times 0.15 \\
\times 0.07\end{array}$ & $\begin{array}{c}0.24 \times 0.08 \\
\times 0.07\end{array}$ & $\begin{array}{c}0.26 \times 0.25 \\
\times 0.12\end{array}$ & $\begin{array}{c}0.16 \times 0.14 \\
\times 0.03\end{array}$ & $\begin{array}{c}0.43 \times 0.08 \times \\
0.07\end{array}$ & $\begin{array}{c}0.51 \times 0.17 \\
\times 0.12\end{array}$ & $0.41 \times 0.12 \times 0.11$ \\
\hline$T(\mathrm{~K})$ & $173(2)$ & $173(2)$ & $173(2)$ & $173(2)$ & $173(2)$ & $173(2)$ & $173(2)$ \\
\hline $\begin{array}{l}\text { crystal } \\
\text { system }\end{array}$ & monoclinic & monoclinic & triclinic & monoclinic & monoclinic & monoclinic & Triclinic \\
\hline space group & $P 2_{1} / c$ & $P 2_{1} / c$ & $P-1$ & $P 2_{1} / m$ & $P 2_{1} / n$ & $P 2{ }_{1} / c$ & $P-1$ \\
\hline$a(\AA)$ & $\begin{array}{l}7.53850 \\
(10)\end{array}$ & $7.3728(12)$ & $7.524(2)$ & $8.2744(4)$ & $3.6912(6)$ & $7.6375(2)$ & $7.01080(10)$ \\
\hline$b(\AA)$ & $\begin{array}{c}8.21730 \\
(10)\end{array}$ & $12.308(2)$ & $8.428(2)$ & $6.8135(4)$ & $14.400(2)$ & 39.0017 (9) & $7.74180(10)$ \\
\hline$c(\AA)$ & 19.2944 (3) & $15.748(3)$ & $12.886(4)$ & $13.9417(8)$ & $11.4516(18)$ & $7.0193(2)$ & $19.7128(4)$ \\
\hline$\alpha\left(^{\circ}\right)$ & 90 & 90 & $73.244(3)$ & 90 & 90 & 90 & $98.6410(10)$ \\
\hline$\beta\left({ }^{\circ}\right)$ & $\begin{array}{c}99.1250 \\
(10)\end{array}$ & $91.199(2)$ & $76.527(3)$ & $104.470(4)$ & 95.8970 (19) & $\begin{array}{c}98.3720 \\
(10)\end{array}$ & $93.7360(10)$ \\
\hline$\gamma\left({ }^{\circ}\right)$ & 90 & 90 & $65.660(3)$ & 90 & 90 & 90 & $99.1600(10)$ \\
\hline$V\left(\AA^{3}\right)$ & $1180.09(3)$ & $1428.8(4)$ & $706.7(3)$ & 761.07 (7) & 605.49 (17) & 2068.60 (9) & $1039.94(3)$ \\
\hline$Z$ & 4 & 4 & 2 & 2 & 2 & 12 & 4 \\
\hline$Z^{\prime}$ & 1 & 1 & 1 & 0.5 & 0.5 & 3 & 2 \\
\hline $\begin{array}{c}\text { wavelength } \\
(\AA ̊)\end{array}$ & 1.541838 & 0.71030 & 0.710730 & 1.541838 & 0.710730 & 1.541838 & 1.541838 \\
\hline $\begin{array}{l}\text { radiation } \\
\text { type }\end{array}$ & $\mathrm{CuK}_{\alpha}$ & $\operatorname{MoK}_{\alpha}$ & $\operatorname{MoK}_{\alpha}$ & $\mathrm{CuK}_{\alpha}$ & $\operatorname{MoK}_{\alpha}$ & $\mathrm{CuK}_{\alpha}$ & $\mathrm{CuK}_{\alpha}$ \\
\hline$\Theta_{\min }\left({ }^{\circ}\right)$ & 4.642 & 2.100 & 1.665 & 5.521 & 2.280 & 2.266 & 2.276 \\
\hline$\Theta_{\max }\left({ }^{\circ}\right)$ & 72.174 & 25.384 & 26.063 & 68.083 & 26.344 & 72.103 & 70.079 \\
\hline $\begin{array}{l}\text { measured } \\
\text { reflections }\end{array}$ & 7228 & 11294 & 12041 & 4098 & 10217 & 12216 & 12061 \\
\hline $\begin{array}{l}\text { independent } \\
\text { reflections }\end{array}$ & 2295 & 2614 & 2791 & 1471 & 1246 & 3995 & 3792 \\
\hline $\begin{array}{c}\text { reflections } \\
\text { used }\end{array}$ & 1819 & 1442 & 2049 & -- & -- & 3494 & 3342 \\
\hline $\begin{array}{l}\text { reflections } \\
\text { with I }>2(I)\end{array}$ & -- & -- & -- & 761 & 1011 & -- & -- \\
\hline$R_{\text {int }}$ & 0.0327 & 0.0714 & 0.0285 & 0.0733 & 0.0360 & 0.0367 & 0.0309 \\
\hline parameters & 172 & 208 & 208 & 130 & 111 & 310 & 310 \\
\hline restraints & 0 & 0 & 0 & 0 & 0 & 0 & 0 \\
\hline largest peak & 0.161 & 0.199 & 0.399 & 0.274 & 0.510 & 0.319 & 0.155 \\
\hline deepest hole & -0.233 & -0.211 & -0.215 & -0.292 & -0.221 & -0.278 & -0.287 \\
\hline GooF & 1.027 & 0.993 & 1.068 & 1.014 & 1.117 & 1.085 & $1 / 043$ \\
\hline$w R_{2}$ (all data) & 0.1133 & 0.1556 & 0.1785 & 0.1941 & 0.2156 & 0.1601 & 0.1289 \\
\hline$w R_{2}$ & 0.1039 & 0.1201 & 0.1606 & 0.1569 & 0.2061 & 0.1565 & 0.1218 \\
\hline$R_{1}$ (all data) & 0.0526 & 0.1135 & 0.0738 & 0.1373 & 0.0826 & 0.0646 & 0.0424 \\
\hline$R_{1}$ & 0.0401 & 0.0526 & 0.0573 & 0.0684 & 0.0720 & 0.0582 & 0.0367 \\
\hline
\end{tabular}


(a)

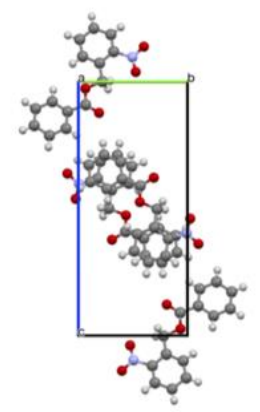

(b)

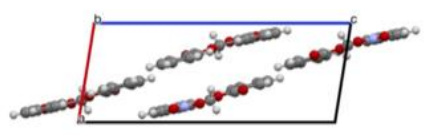

(c)

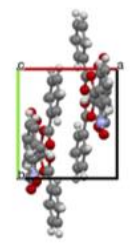

Figure S17. Unit cell of 2-nitrobenzyl benzoate (1). (a) Unit cell as viewed down the crystallographic a-axis. (b) Unit cell as viewed down the crystallographic b-axis. (c) Unit cell as viewed down the crystallographic c-axis.

(a)

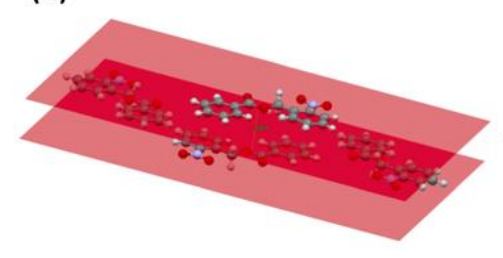

(b)

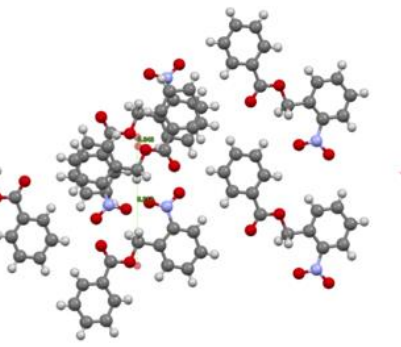

(c)

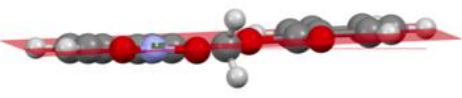

Figure S18. Relevant measurements within the unit cell of 2-nitrobenzyl benzoate (1).

(a)

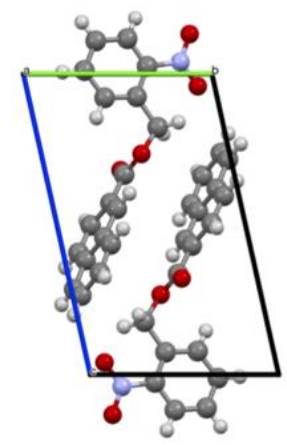

(b)

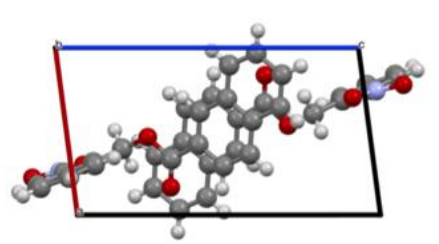

(c)

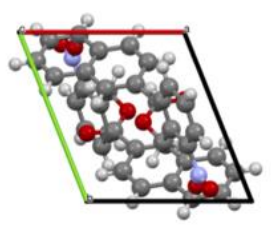

Figure S19. Unit cell of 2-nitrobenzyl 2-naphthoate (2). (a) Unit cell as viewed down the crystallographic a-axis. (b) Unit cell as viewed down the crystallographic b-axis. (c) Unit cell as viewed down the crystallographic c-axis. 

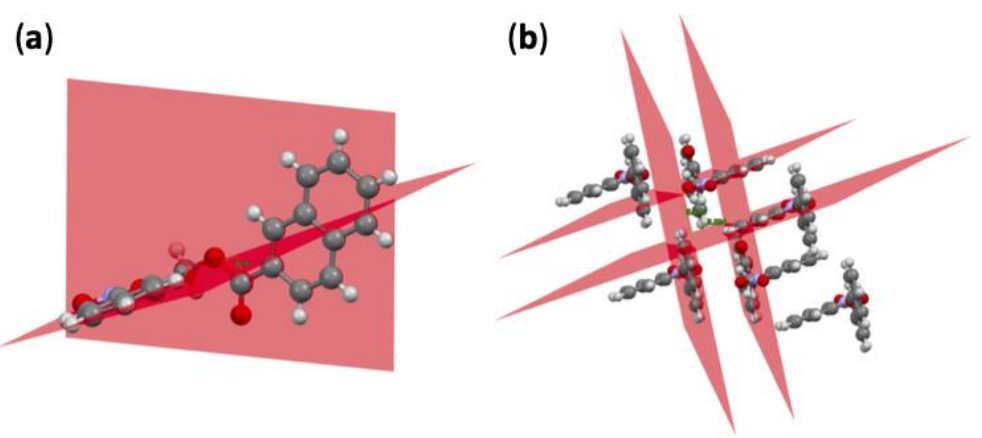

Figure S20. Relevant measurements within the unit cell of 2-nitrobenzyl 2-naphthoate (2).
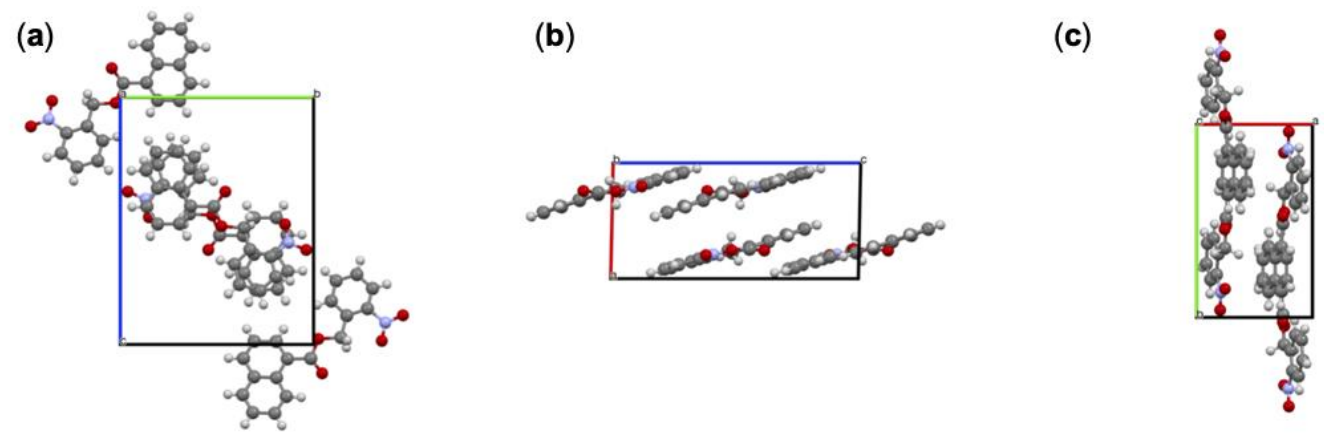

Figure S21. Unit cell of 2-nitrobenzyl 1-naphthoate (3). (a) Unit cell as viewed down the crystallographic a-axis. (b) Unit cell as viewed down the crystallographic b-axis. (c) Unit cell as viewed down the crystallographic c-axis.
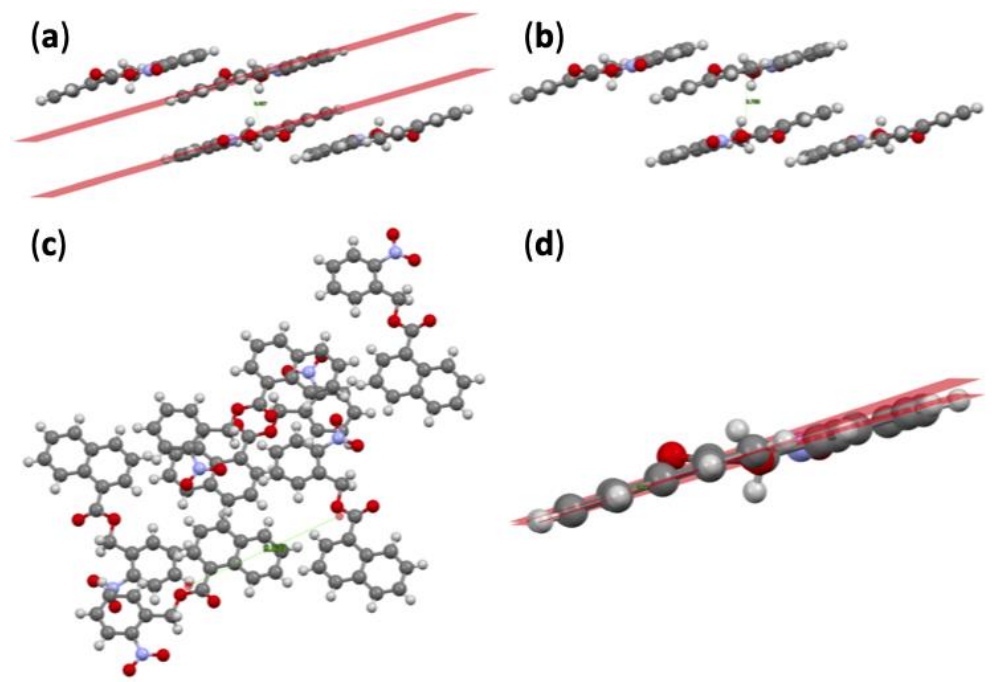

(d)

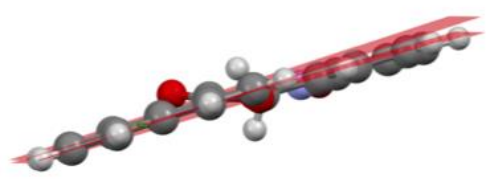

Figure S22. Relevant measurements within the unit cell of 2-nitrobenzyl 1-naphthoate (3). 
(a)

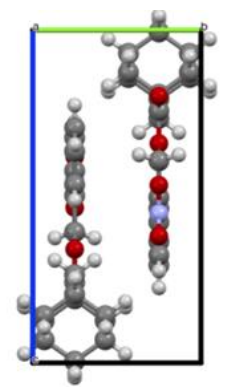

(b)

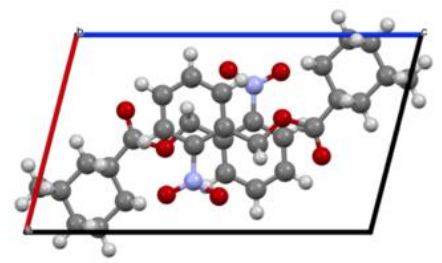

(c)

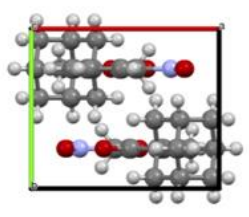

Figure S23. Unit cell of 2-nitrobenzyl 1-adamantane carboxylate (4). (a) Unit cell as viewed down the crystallographic a-axis. (b) Unit cell as viewed down the crystallographic b-axis. (c) Unit cell as viewed down the crystallographic c-axis.

(a)

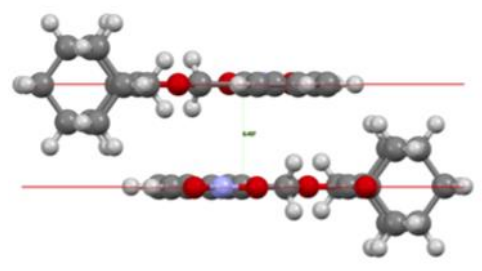

(b)

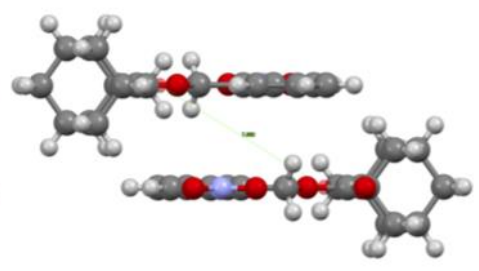

(c)

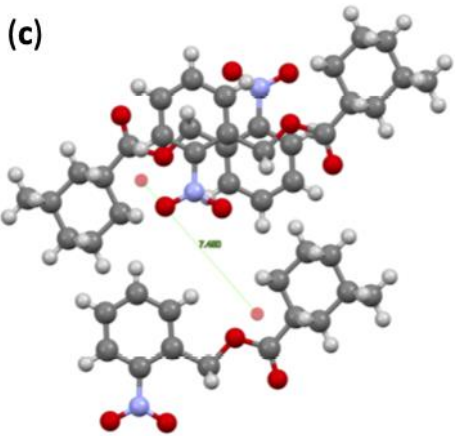

Figure S24. Relevant measurements within the unit cell of 2-nitrobenzyl 1-adamantane carboxylate (4).

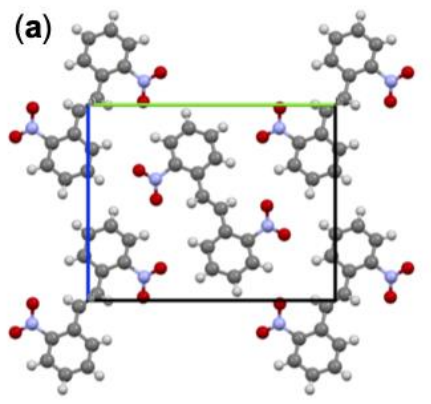

(b)

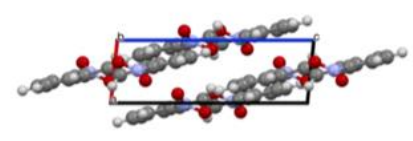

(c)

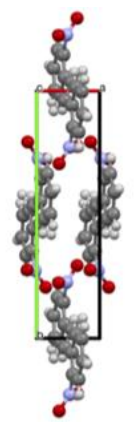

Figure S25. Unit cell of 1,2-bis(2-nitrophenyl)ethane (5). (a) Unit cell as viewed down the crystallographic a-axis. (b) Unit cell as viewed down the crystallographic b-axis. (c) Unit cell as viewed down the crystallographic c-axis. 
(a)

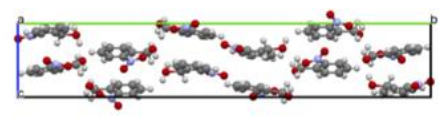

(b)

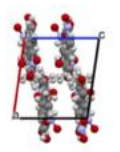

(c)

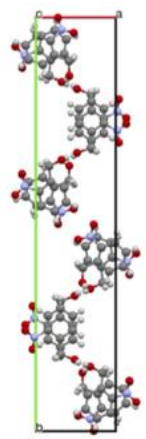

Figure S26. Unit cell of monoclinic 2-nitrobenzyl alcohol (6). (a) Unit cell as viewed down the crystallographic a-axis. (b) Unit cell as viewed down the crystallographic b-axis. (c) Unit cell as viewed down the crystallographic c-axis.

(a)

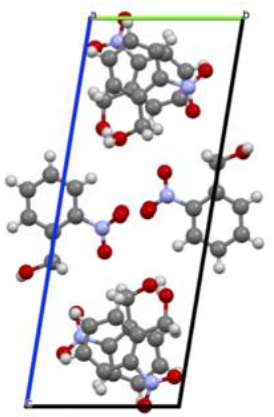

(b)

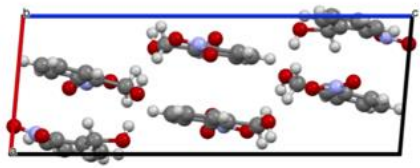

(c)

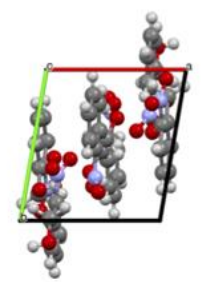

Figure S27. Unit cell of triclinic 2-nitrobenzyl alcohol (7). (a) Unit cell as viewed down the crystallographic a-axis. (b) Unit cell as viewed down the crystallographic b-axis. (c) Unit cell as viewed down the crystallographic c-axis.

(a)

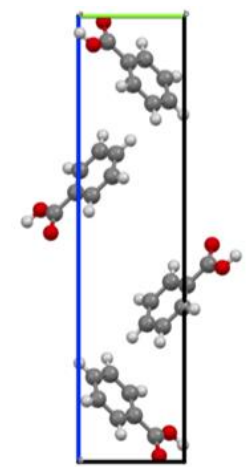

(b)

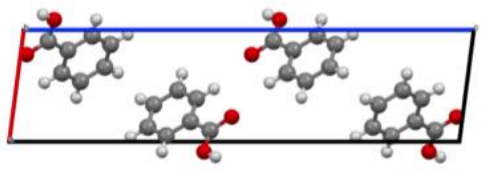

(c)

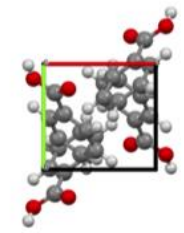

Figure S28. Unit cell of benzoic acid. (a) Unit cell as viewed down the crystallographic a-axis. (b) Unit cell as viewed down the crystallographic b-axis. (c) Unit cell as viewed down the crystallographic c-axis. 
(a)

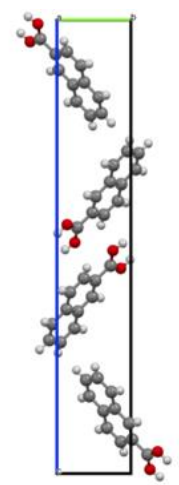

(b)

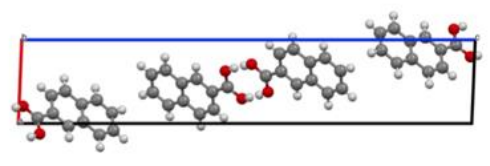

(c)

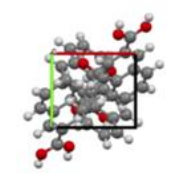

Figure S29. Unit cell of 2-naphthoic acid. (a) Unit cell as viewed down the crystallographic a-axis. (b) Unit cell as viewed down the crystallographic b-axis. (c) Unit cell as viewed down the crystallographic c-axis.

(a)

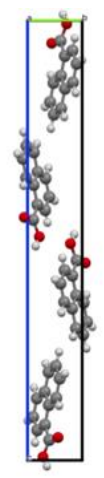

(b)

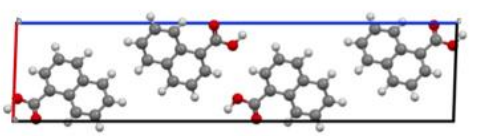

(c)

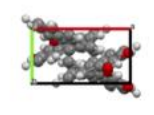

Figure S30. Unit cell of 1-naphthoic acid. (a) Unit cell as viewed down the crystallographic a-axis. (b) Unit cell as viewed down the crystallographic b-axis. (c) Unit cell as viewed down the crystallographic c-axis.

(a)

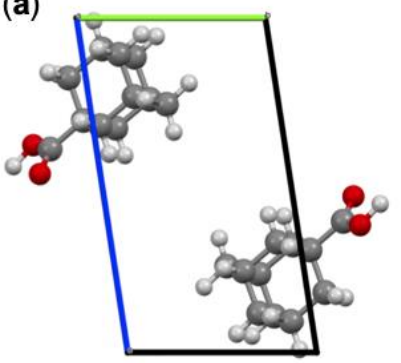

(b)

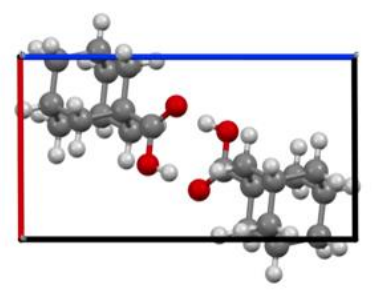

(c)

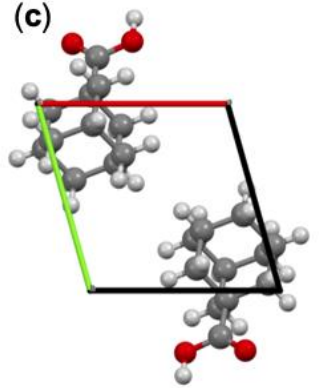

Figure S31. Unit cell of adamantane-1-carboxylic acid. (a) Unit cell as viewed down the crystallographic a-axis. (b) Unit cell as viewed down the crystallographic b-axis. (c) Unit cell as viewed down the crystallographic c-axis.

\section{SVI. Hirshfeld Surface Calculations and Fingerprint Plot Analysis}

Hirshfeld surface analysis was conducted using CrystalExplorer software Version: $17.5 .^{6-}$

${ }^{10}$ Crystallographic information files (CIFs) for each material were imported into the program, and 
the Surface Generation dialogue box was used to calculate a Hirshfeld surface (Property: none, Resolution: very high). From the calculated surface, the Display Fingerprint Plot option was selected to visualize the fingerprint plot. Within the fingerprint plot window, the option to filter the plot by element was selected. Each combination of "inside" and "outside" atom was selected, and the percent surface area for that combination was recorded (Figure S32). Standard software options were used to save/export the data.

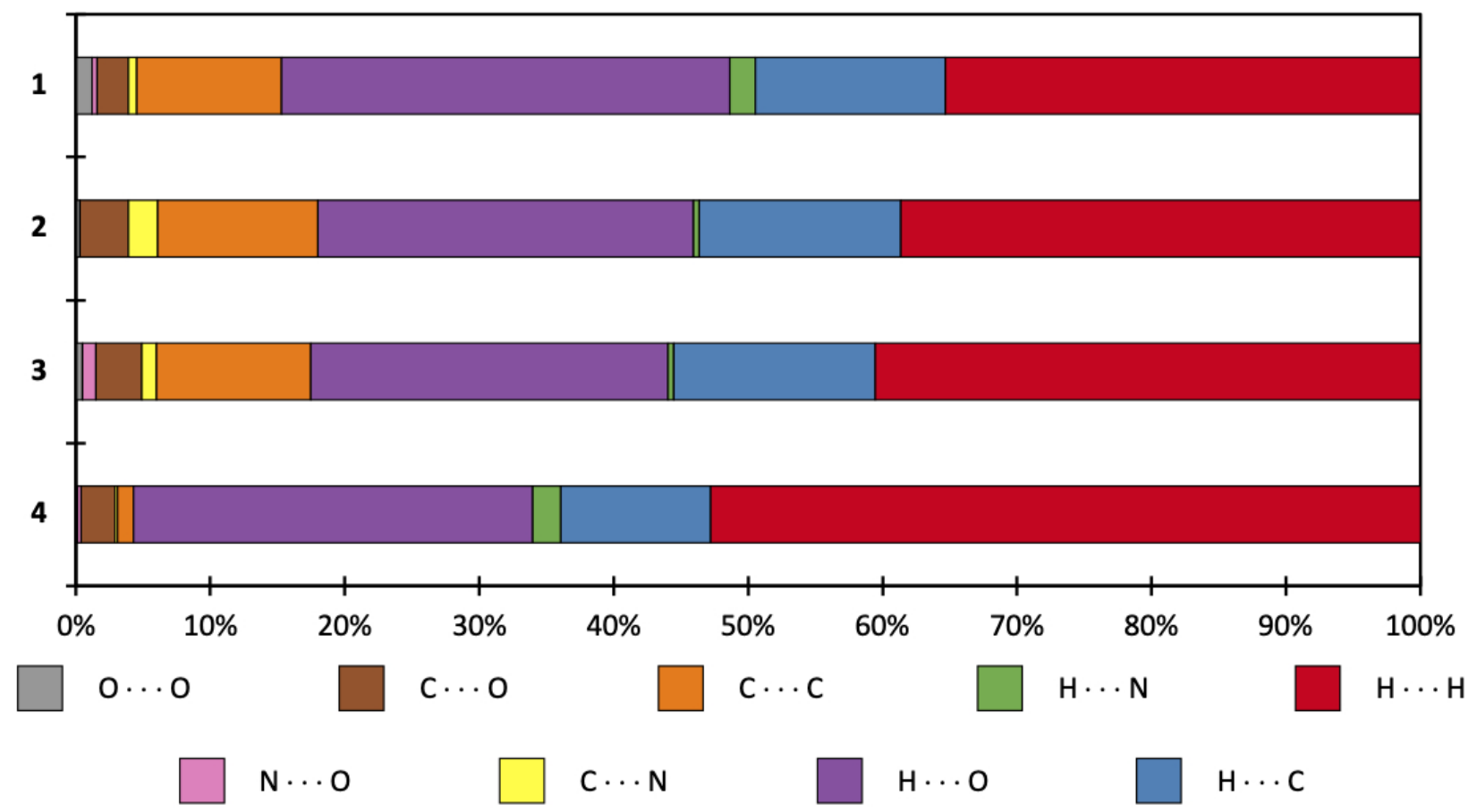

Figure S32. Quantification of the intermolecular interactions present within each photoresponsive crystalline material. Percentages of each interaction were calculated using Hirshfeld Surface Analysis in CrystalExplorer.

\section{SVII. Calculation of Crystal Structure Correlation Coefficients}

Correlation coefficients were calculated following the structural genetic fingerprinting procedure of Spackman and coworkers. ${ }^{11}$ Intensity data from the fingerprint plot of each material analyzed was exported from CrystalExplorer as a series of $(x, y$, intensity) values, then imported 
following script:

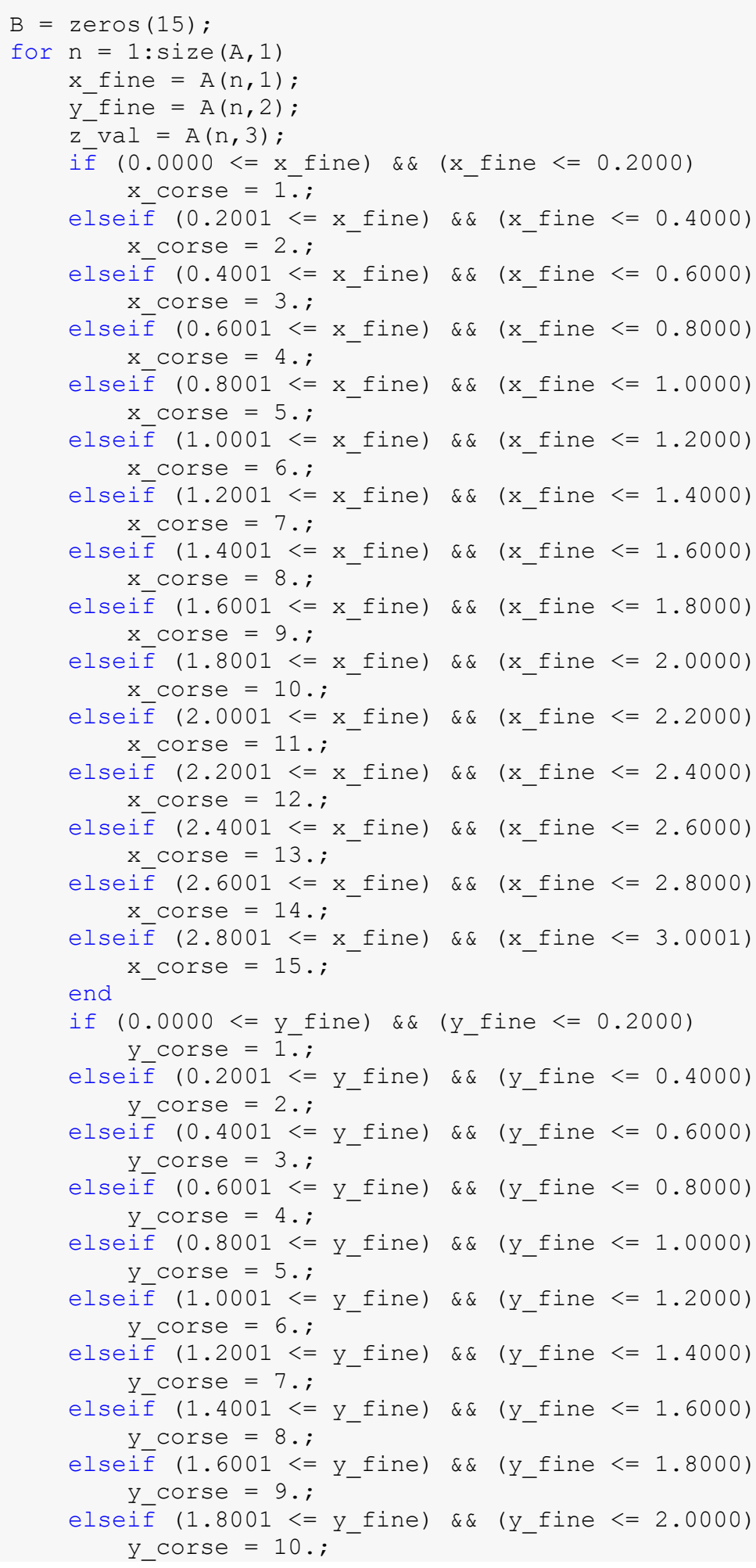




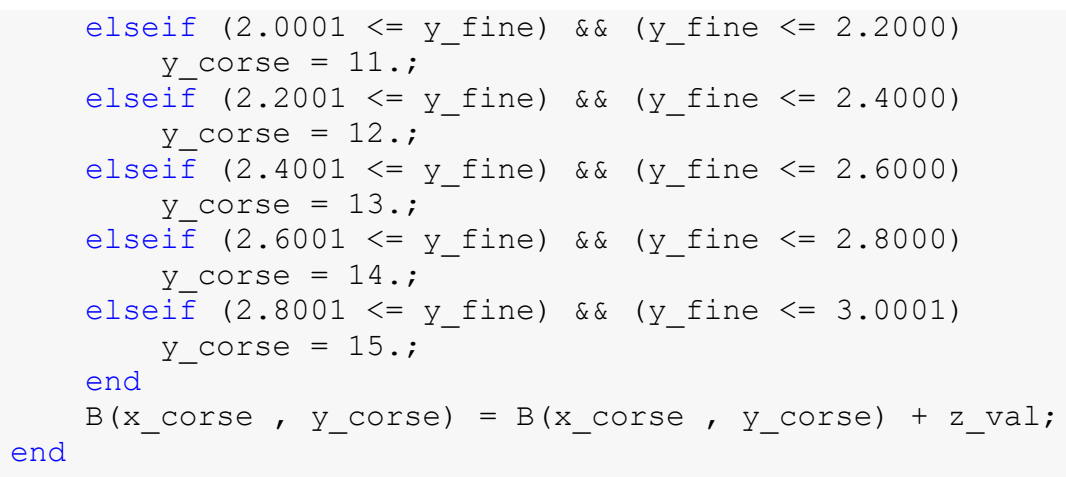

The resulting matrix, $B$, was a $15 \times 15$ matrix containing the binned data, which was then saved in the workspace under a different variable name. Once the data from all relevant crystal structures was processed, the correlation coefficient between two matrices, matrix_1 and matrix_2, was calculated by "corrcoef (matrix_1, matrix_2);". When comparing two matrices, rows and columns that were identical in both were ignored to enhance the difference.

\section{SVIII. Melt-Bonding Quartz Substrates with Photoresponsive Crystalline}

\section{Adhesives}

For melt-bonding of quartz microscope slides, the hotplate and substrates were preheated at $\sim 10{ }^{\circ} \mathrm{C}$ above the melting point of the adhesive to ensure complete and rapid melting. Melt-bonding temperatures were $130,110,135$, and $150{ }^{\circ} \mathrm{C}$, for $1-4$, respectively. Once both substrates had preheated, $60 \mathrm{mg}$ adhesive powder was placed on the bottom substrate, and the top substrate was then added allowing for a $20 \times 25 \mathrm{~mm}^{2}$ bonded overlap. While in the liquid phase, the adhesive was allowed to reach its thermodynamic and/or kinetic equilibrium with respect to surface wetting of the adherends and film thickness. Once equilibrium had been achieved, the assembly was removed from hotplate and placed on a lab jack that acted as a cooling stage. As the melted adhesive cooled it fused and bonded the substrates. 


\section{SIX. Obtaining and Analyzing Micrographs of Polycrystalline Films of}

\section{Photoresponsive Adhesives}

A Keyence High-Definition Light Microscope was used to image the overlap joint of each sample before and after mechanical testing. In addition to the microscope's built-in light source, an auxiliary light was used to illuminate the assemblies, and provide enhanced contrast and visibility. The built-in light source was directly above the sample, normal to the surface of the top substrate. The auxiliary light source was directed at a $\sim 15^{\circ}$ angle relative to the surface of the assembly to assist in discerning grain boundaries and voids in the polycrystalline films. A handheld UV lamp (Fisher Scientific, Supplier No.: UVP50021112) was placed behind the sample such that the darkly tinted window minimized light reflection. The same lighting conditions were used to image both substrates of each sheared assembly. Average crystal grain size was calculated according to ASTM E112-13. ${ }^{13}$

Optical micrographs were processed in Image J 5.0 to determine the surface area of the polycrystalline adhesive film. The scale was set within each image using a scale bar imaged next to the sample. The Threshold plugin enabled the contrast between crystallites and voids to be digitally optimized. Representative optical light micrographs of 1-4 before and after shearing are presented in Figures S33-S36. 
(a)<smiles>O=Nc1ccccc1COC(=O)c1ccccc1</smiles>

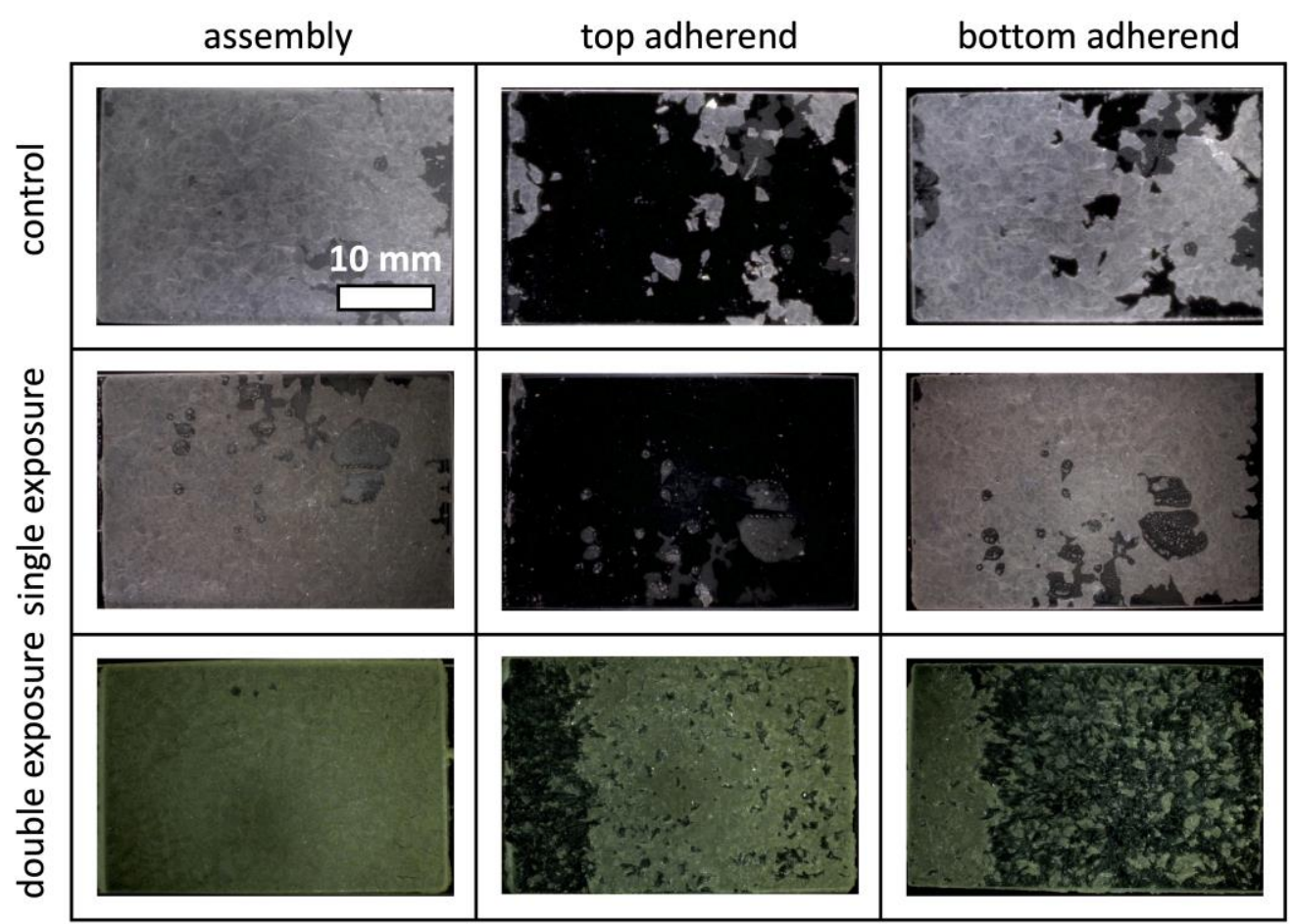

(b)

$2.5 \mathrm{~mm}$

Figure S33. Representative optical light micrographs of 2-nitrobenzyl benzoate (1). (a) Optical micrographs were obtained of the bonded assembly before fracture (assembly) and of both adherends after fracture. (b) Enlarged image of the polycrystalline film used to quantitate crystal grain size. 
(a)<smiles>O=C(OCc1ccccc1[N+](=O)[O-])c1ccc2ccccc2c1</smiles>

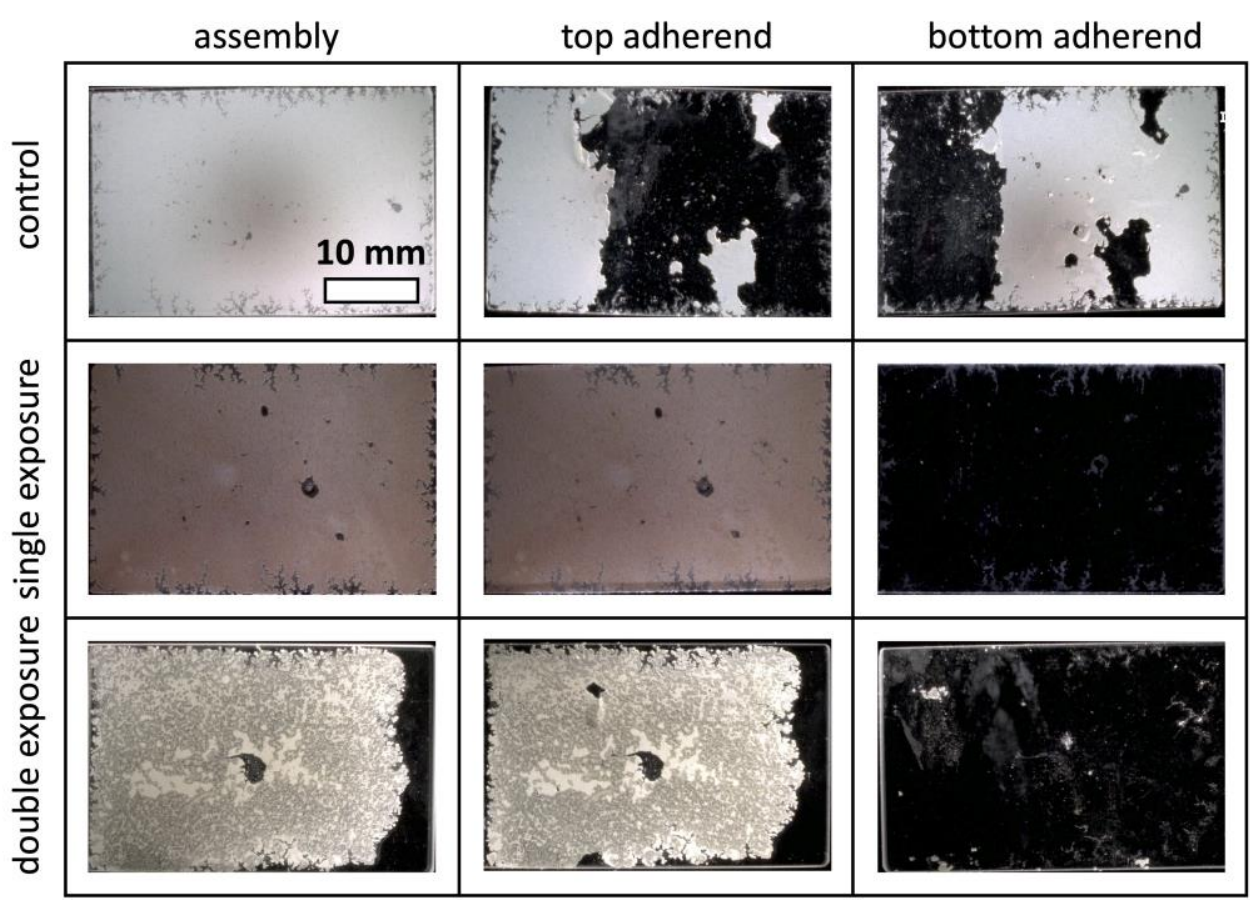

(b)

Figure S34. Representative optical light micrographs of 2-nitrobenzyl 2-naphthoate (2). (a) Optical micrographs were obtained of the bonded assembly before fracture (assembly) and of both adherends after fracture. (b) Enlarged image of the polycrystalline film used to quantitate crystal grain size. 

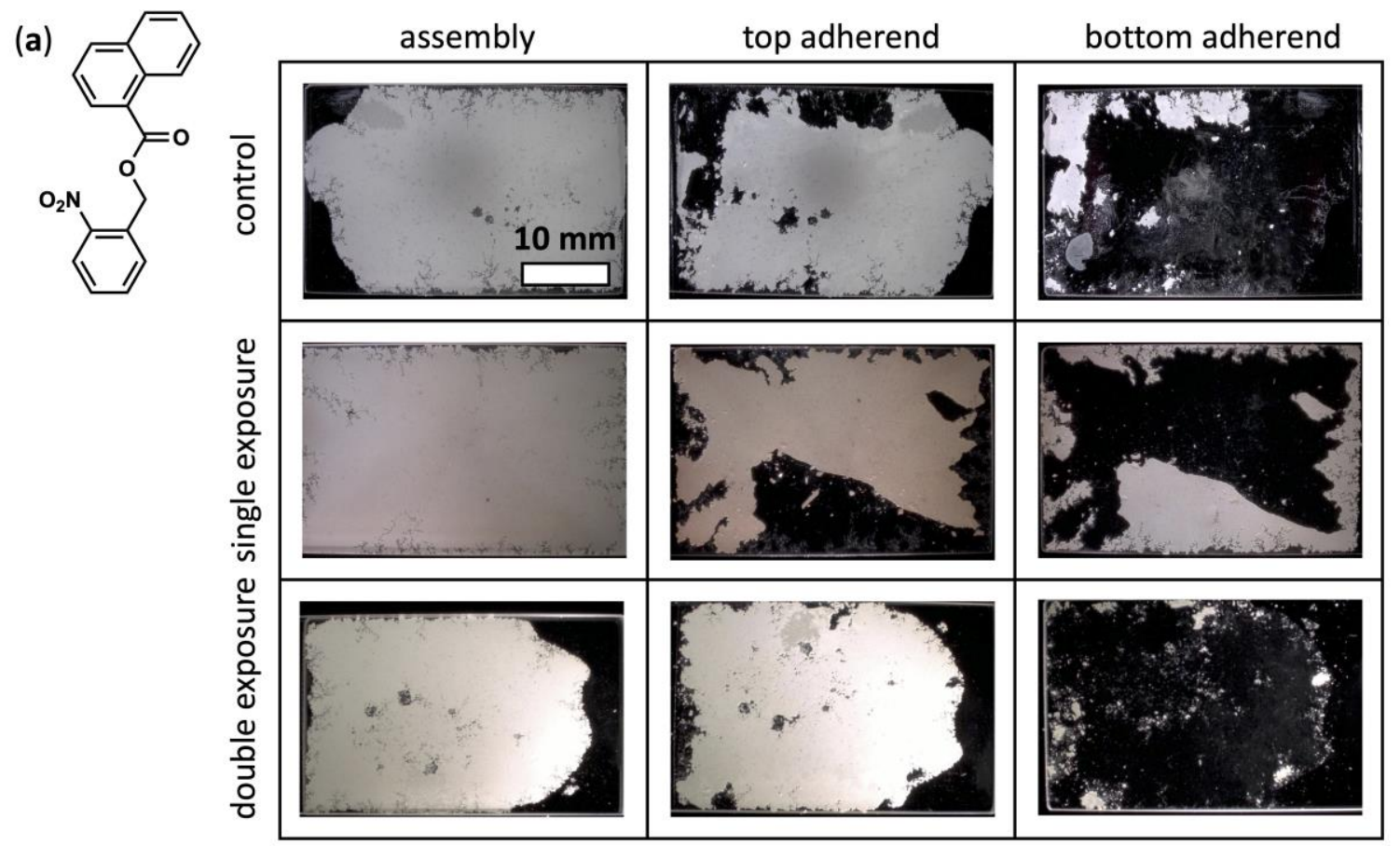

(b)

Figure S35. Representative optical light micrographs of 2-nitrobenzyl 1-naphthoate (3). (a) Optical micrographs were obtained of the bonded assembly before fracture (assembly) and of both adherends after fracture. (b) Enlarged image of the polycrystalline film used to quantitate crystal grain size. 
(a)<smiles>O=C(OCc1ccccc1[N+](=O)[O-])C12CC3CC(CC(C3)C1)C2</smiles>
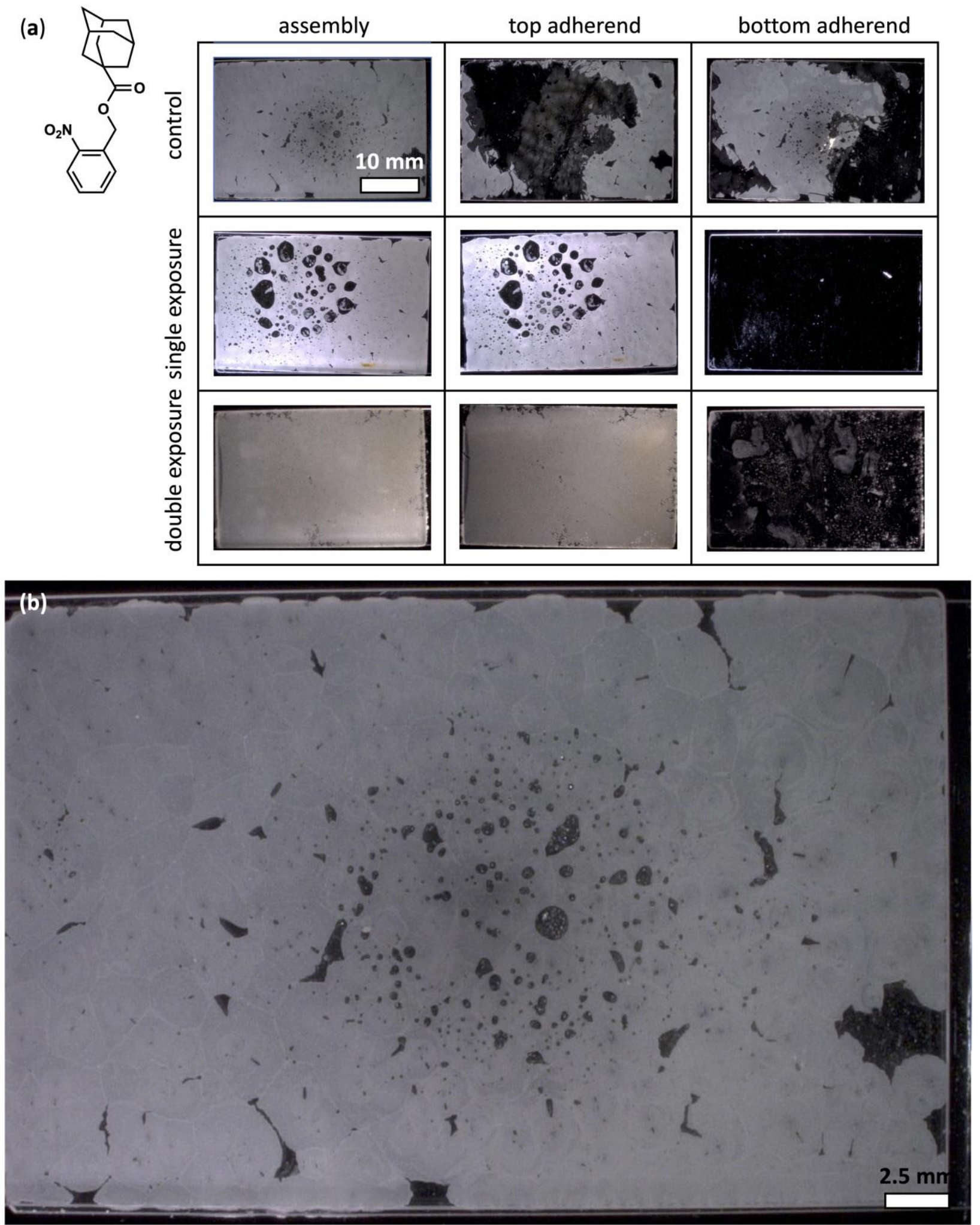

Figure S36. Representative optical light micrographs of 2-nitrobenzyl 1-adamantane carboxylate (4). (a) Optical micrographs were obtained of the bonded assembly before fracture (assembly) and of both adherends after fracture. (b) Enlarged image of the polycrystalline film used to quantitate crystal grain size. 


\section{SX. Shear Testing of the Overlap Joint for Adhesive Measurements}

We used the same shearing protocol in this study as we have in our earlier reports, which is based on the ASTM D-1002-10 standard. ${ }^{14-16}$ Generally, samples were loaded into pneumatic clamps attached to an Instron 5544and sheared at a constant loading rate of $30 \mathrm{~N} \mathrm{~min}^{-1}$. Force and extension data were recorded on Instron's Bluehill software and analyzed in Microsoft Excel. During analysis, an instrumental artifact where slack in the testing rig is recorded as extra extension was removed. Due to inherent variation between samples, we report the parameter stress, $\tau$ (Equation 1). Stress $(\mathrm{kPa})$ is the ratio of maximum force withstood before joint failure (N) to the area of the polycrystalline adhesive film $\left(\mathrm{cm}^{2}\right)$.

$$
\tau\left(N \mathrm{~cm}^{-2}\right)=\frac{\text { maximum force before failure }(N)}{\text { surface area of adhesive layer }\left(\mathrm{cm}^{2}\right)}
$$

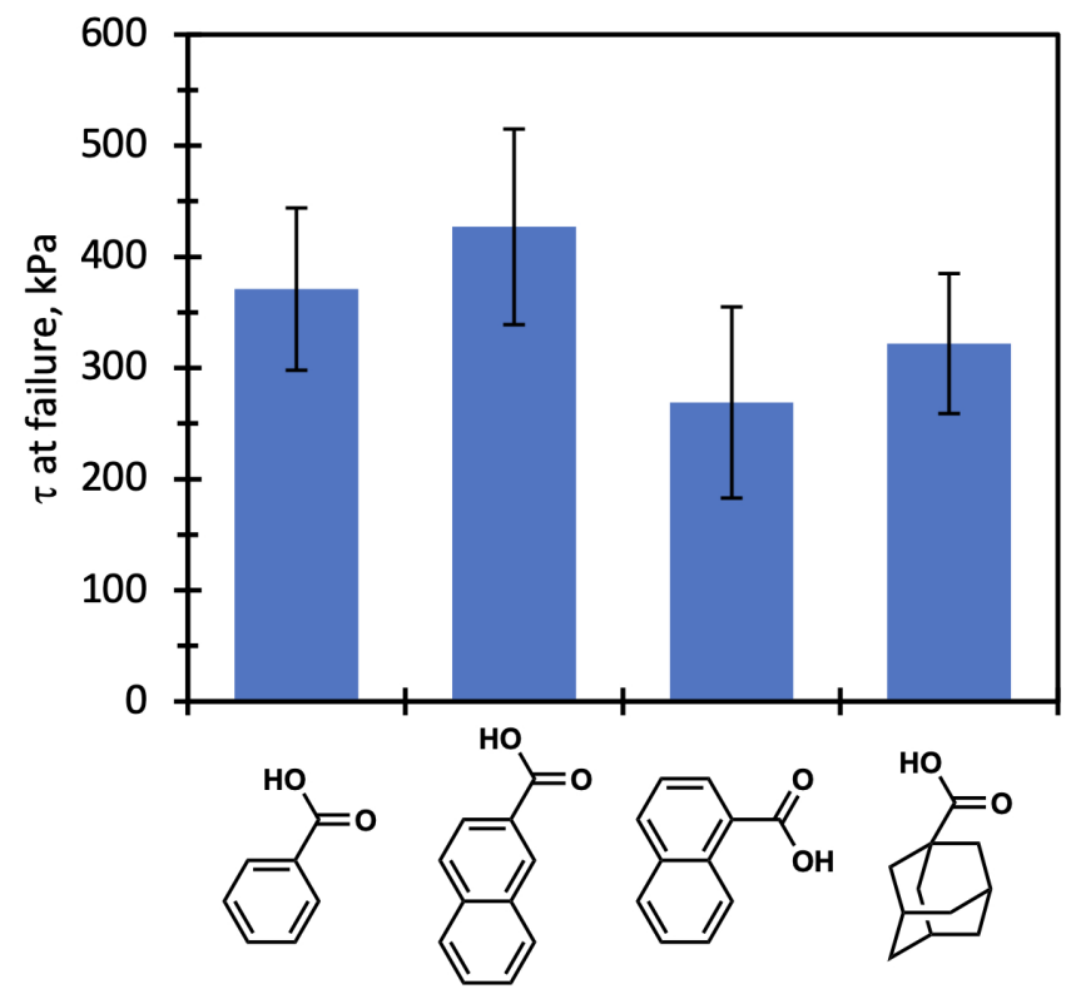

Figure S37. Shear strength of expected photoproducts. Irradiation of compounds 1-4 is expected to produce the benzoic acid, 2-naphthoic acid, 1-naphthoic acid, and adamantane-1-carboxylic acid, respectively.

SXI. Powder X-Ray Diffraction and Calculation of Preferential Orientation 
Representative samples of sheared assemblies of 1-4, benzoic acid, 2-naphthoic acid, 1-naphthoic acid, and adamantane-1-carboxylic acid were analyzed via PXRD. After shearing both adherends, along with the polycrystalline film that remained adhered to it, were analyzed. Each spectrum was collected over the range $2-90^{\circ} 2 \theta$, with a step size of $0.02^{\circ}$ and a scan rate of $10 \mathrm{~min}^{-1}$. Mercury software (Version 4.3.1) was used to calculate ideal PXRD spectra from input CIF files. ${ }^{17-22}$ Comparison of experimental and calculated spectra enabled quantification of preferential orientation due to melt-bonding.

Preferential orientation was calculated from the relative peak intensities in the experimental and calculated spectra. Five prevalent Bragg planes were included in the calculation for each material: the two most intense peaks in the experimental spectrum, and the three nextmost intense peaks in the calculated spectrum. The maximum intensity of each peak was used to represent the entire peak. Differences in the relative peak intensities, as calculated by Equation 2, were interpreted as melt-bonding induced preferential orientation. The relative distribution of prominent Bragg planes before bonding and after bonding and visualizations of the two most preferred planes of 1-4, benzoic acid, 2-naphthoic acid, 1-naphthoic acid, and adamantane-1-carboxylic acid are presented in Figures S38-S45.

$$
\text { relative peak intensity }(\%)=\frac{\text { intensity of selected peak }}{\text { sum intensity of all peaks }}
$$




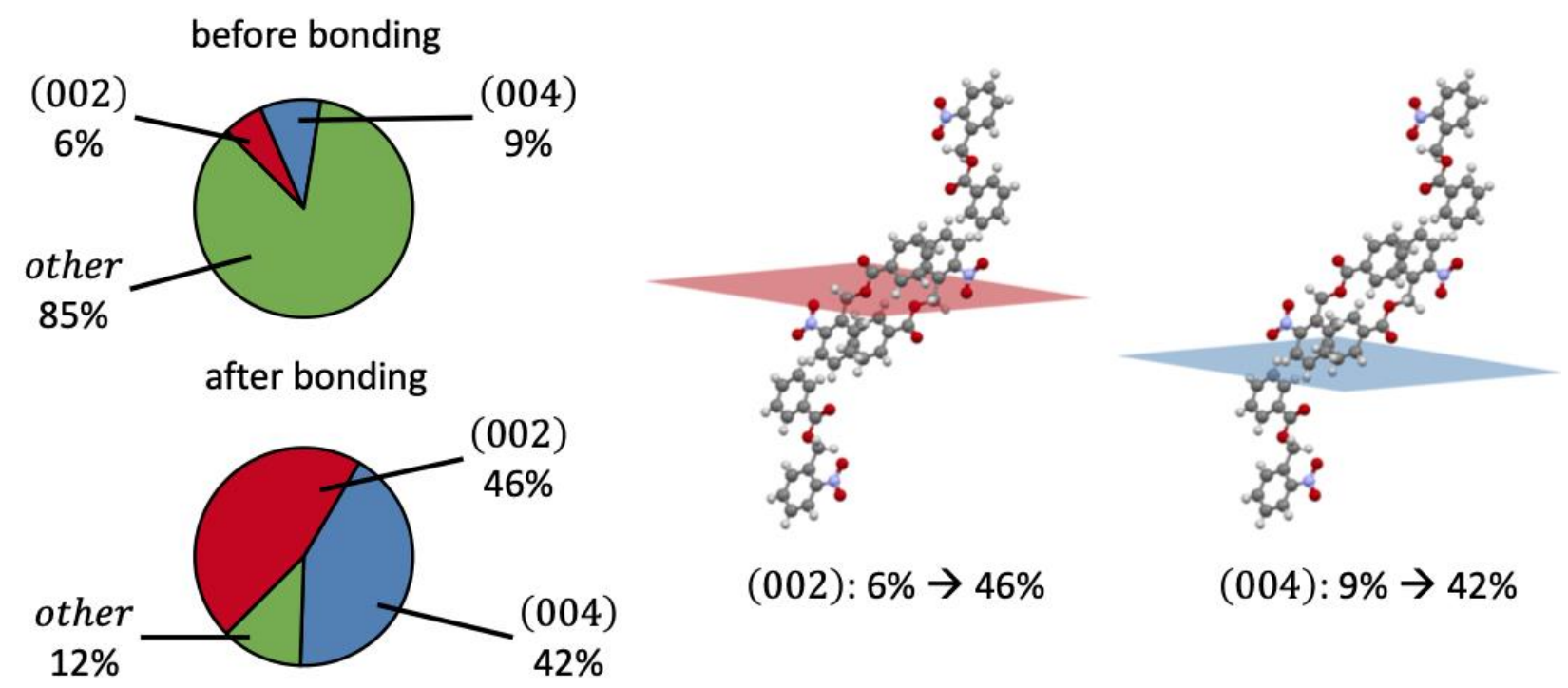

Figure S38. Preferential orientation of 2-nitrobenzyl benzoate (1) as induced by melt-bonding.

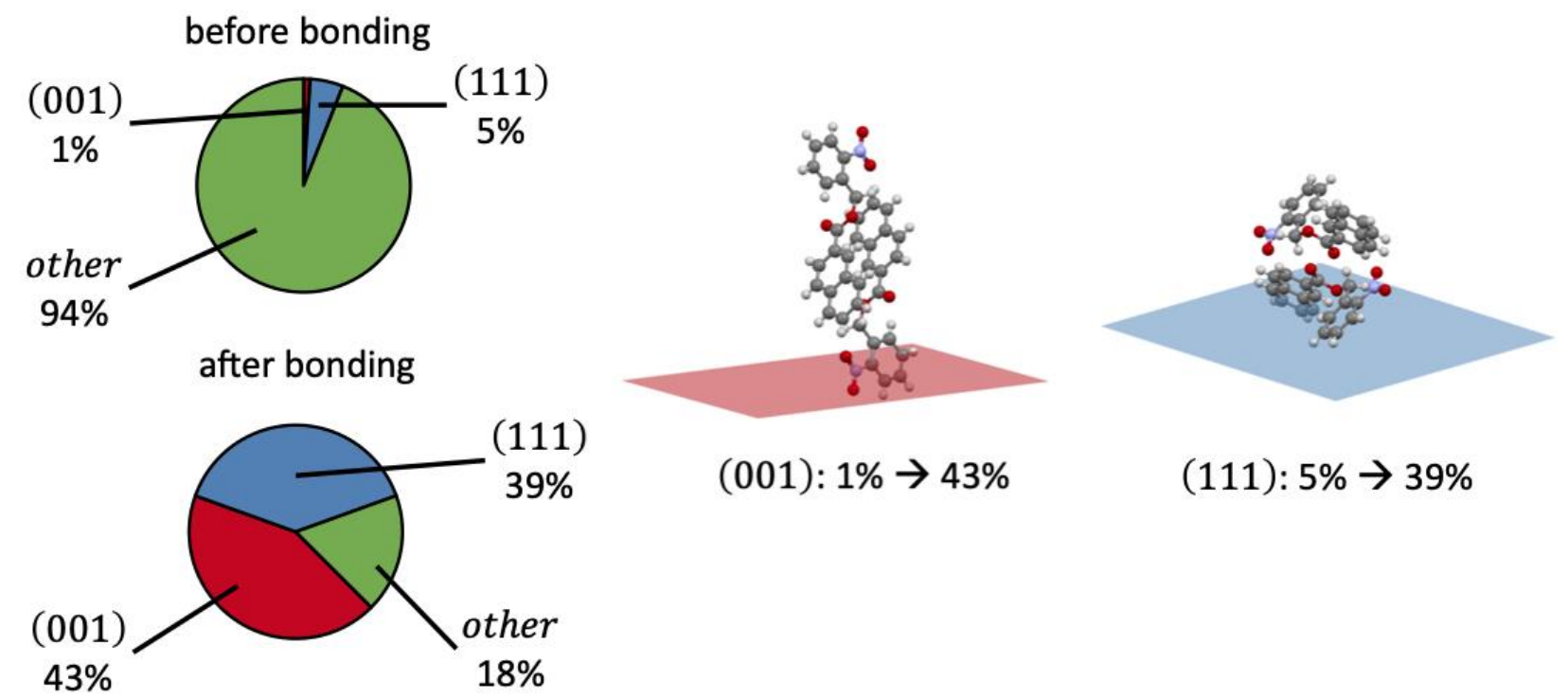

Figure S39. Preferential orientation of 2-nitrobenzyl 2-naphthoate (2) as induced by melt-bonding. 


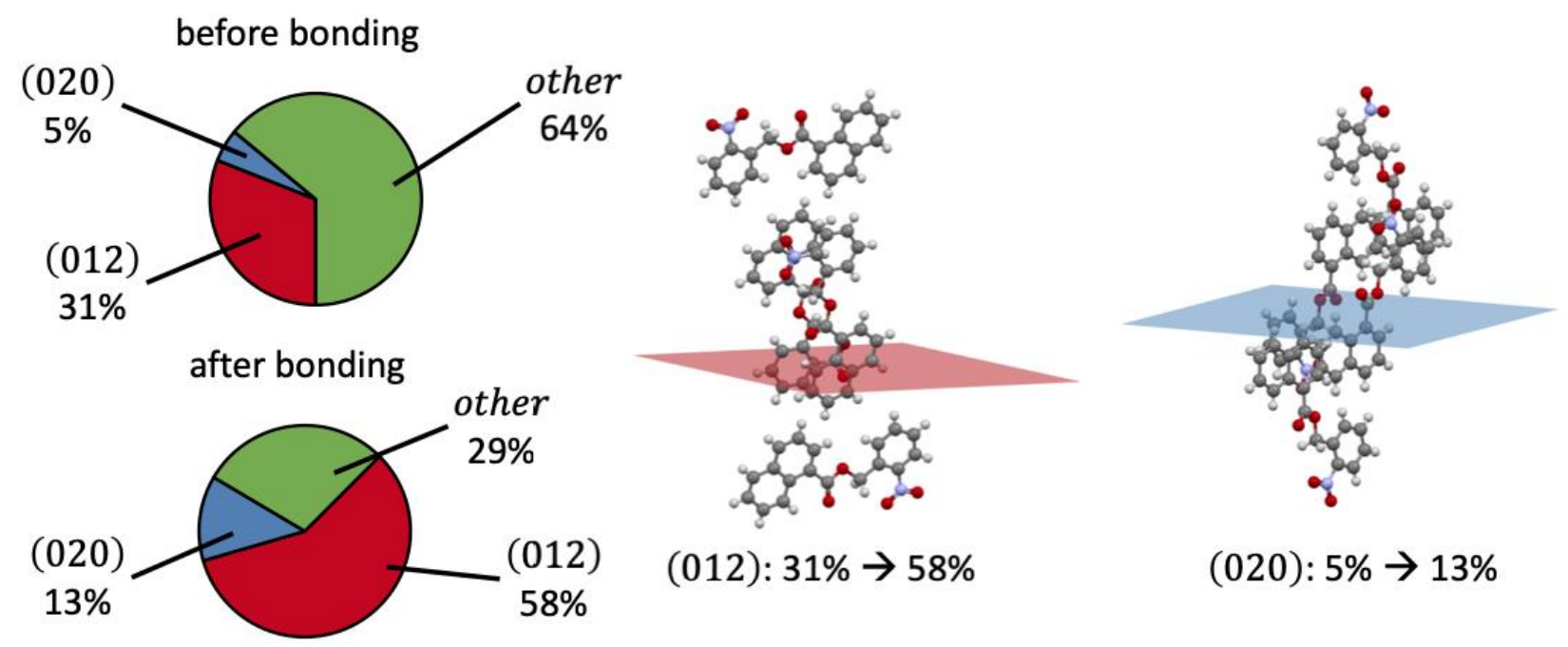

Figure S40. Preferential orientation of 2-nitrobenzyl 1-naphthoate (3) as induced by melt-bonding.

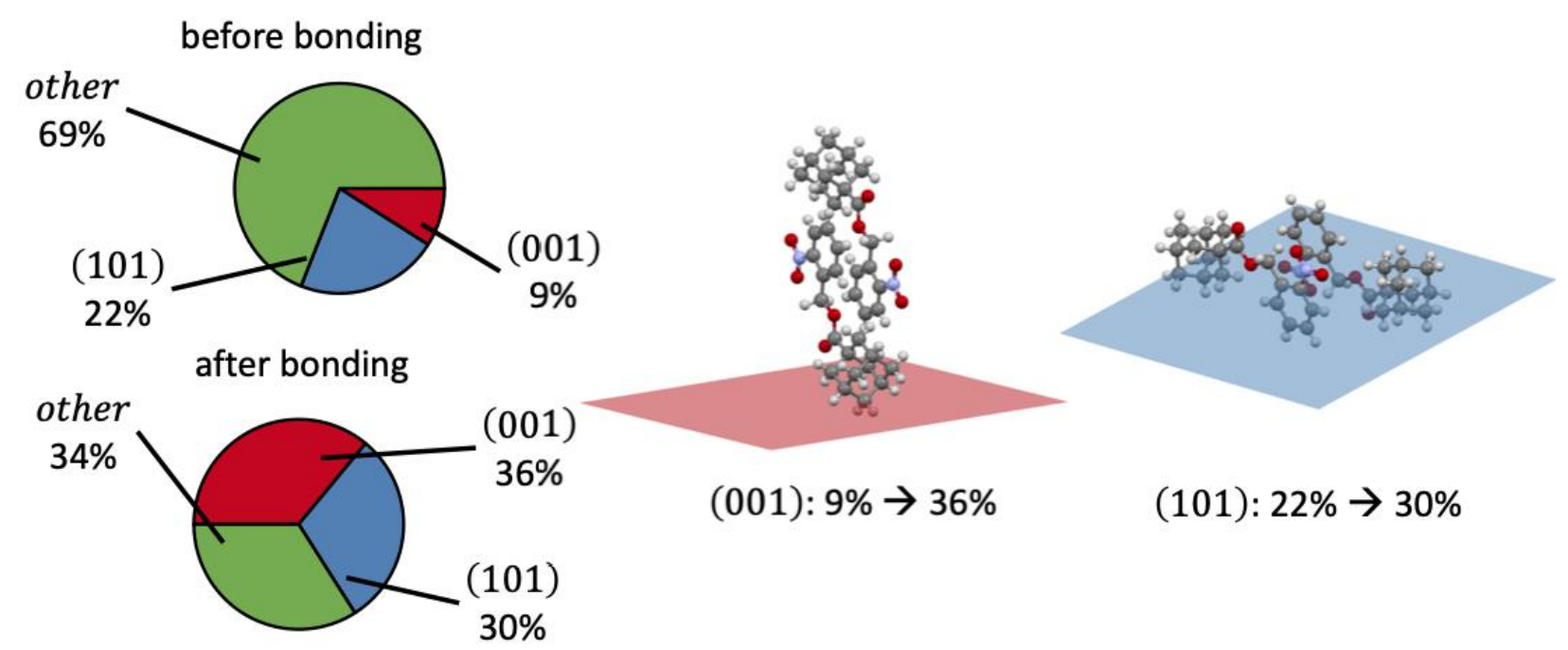

Figure S41. Preferential orientation of 2-nitrobenzyl 1-adamantane carboxylate (4) as induced by meltbonding. 


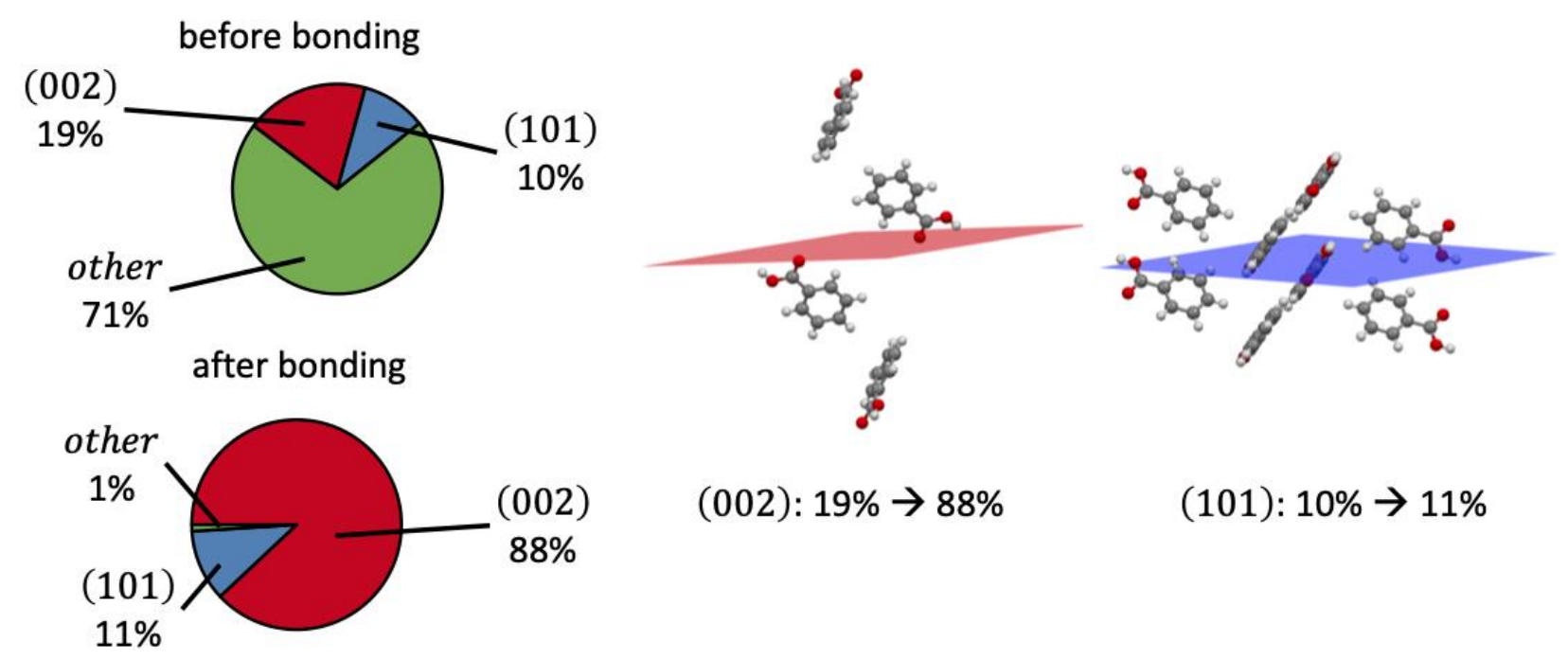

Figure S42. Preferential orientation of benzoic acid as induced by melt-bonding.
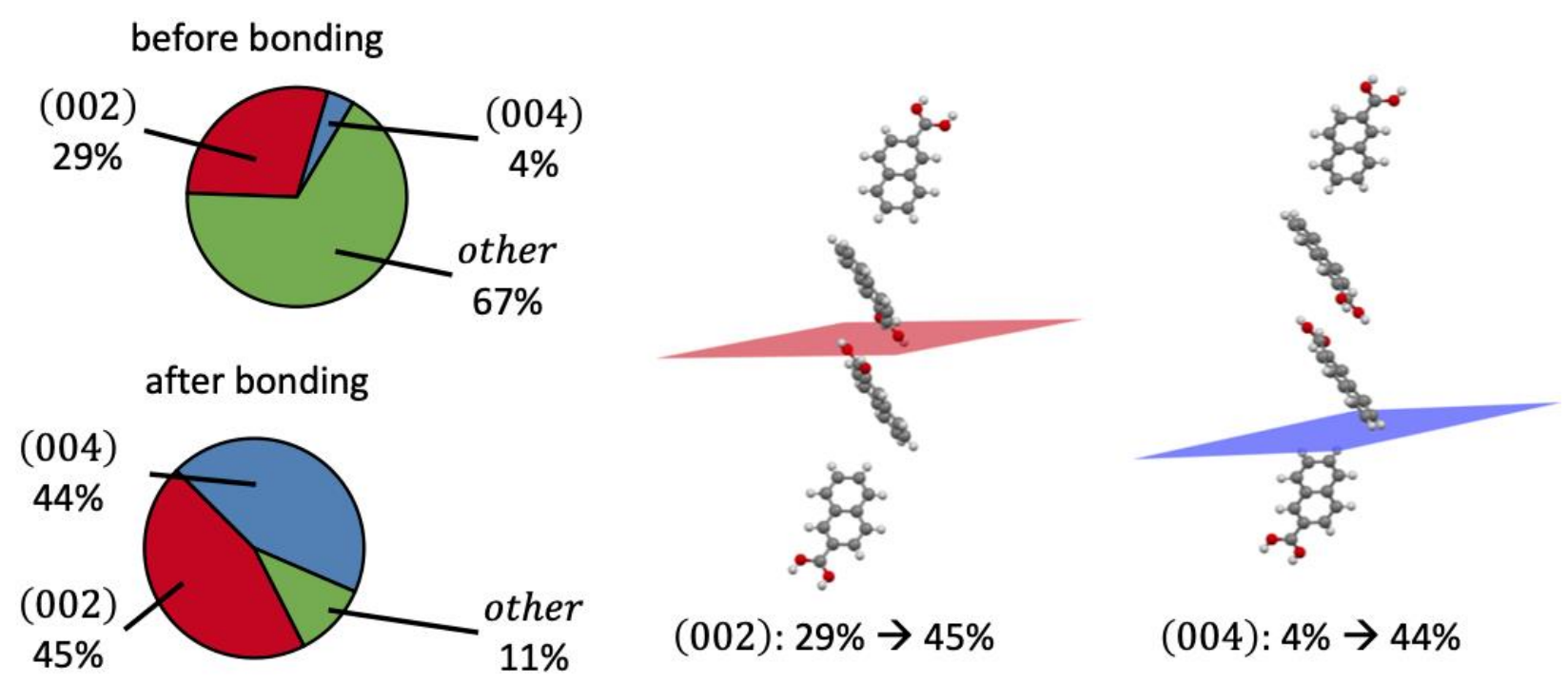

Figure S43. Preferential orientation of 2-naphthoic acid as induced by melt-bonding. 


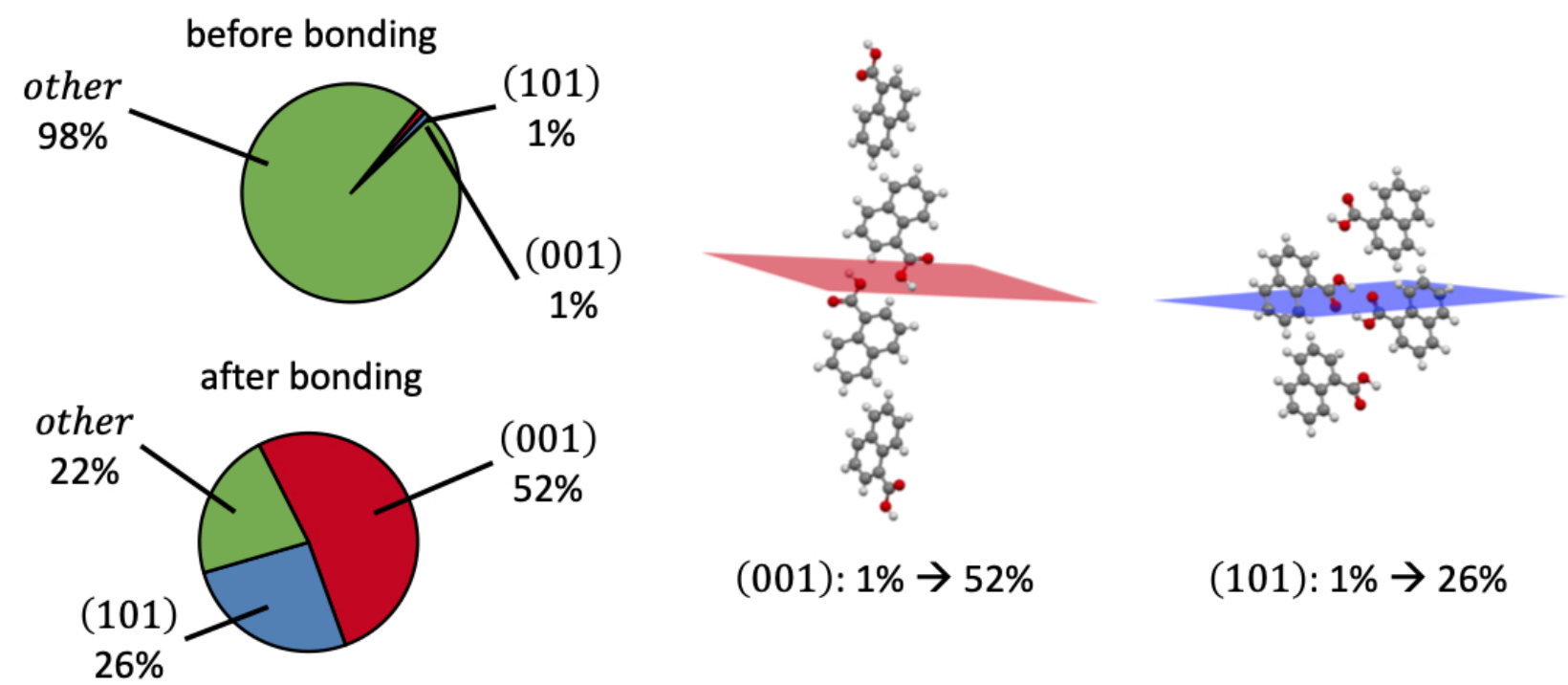

Figure S44. Preferential orientation of 1-naphthoic acid as induced by melt-bonding.

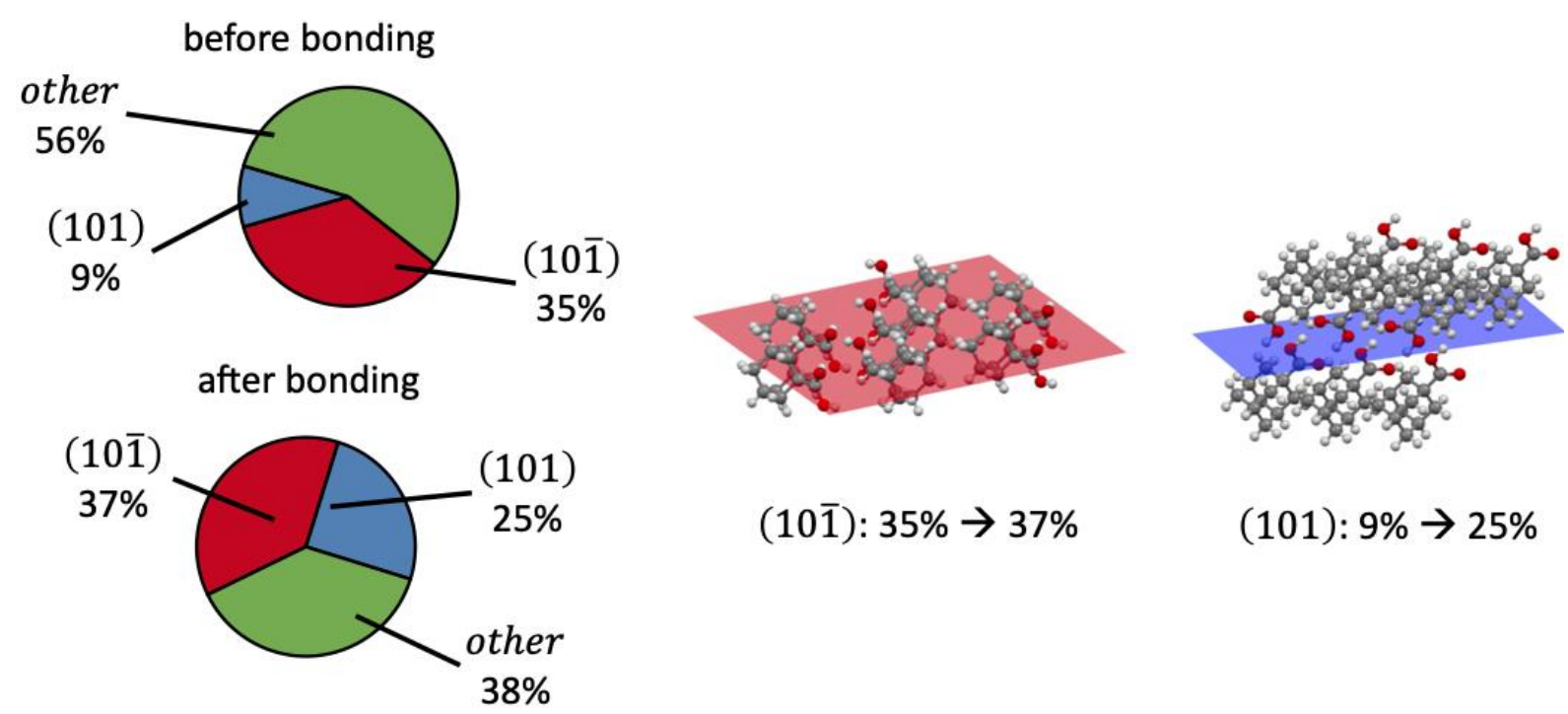

Figure S45. Preferential orientation of adamantane-1-carboxylic acid as induced by melt-bonding.

\section{SXII. UV/Vis Spectroscopy of Photoresponsive Crystalline Adhesives}

Materials 1-4 were analyzed via UV/Vis spectroscopy in both the solution and solid state using a Jasco V570 UV-Visible Spectrophotometer. For solution-state studies, a $1 \mathrm{mM}$ solution of each material in dichloromethane was prepared. Serial dilutions were then utilized to generate solutions in a range of concentrations. Each sample was scanned from $\lambda=800-200 \mathrm{~nm}$ in absorbance mode with a step size of $1 \mathrm{~nm}$ and a scan rate of $100 \mathrm{~nm} \mathrm{~min} \mathrm{~m}^{-1}$ using a quartz cuvette 
(VWR International, Supplier No.: 414004-055, path length $10 \mathrm{~mm}$ ). Each scan was background subtracted against pure dichloromethane, exported, and analyzed in Origin Pro 2020. Concentrations of $50,6.25,25$, and $50 \mu \mathrm{M}$ were found to be on scale (i.e., the maximum absorbance value was less than 1) for 1-4, respectively. The absorbance spectra of 1-4 are presented in Figures S46-S49, respectively.

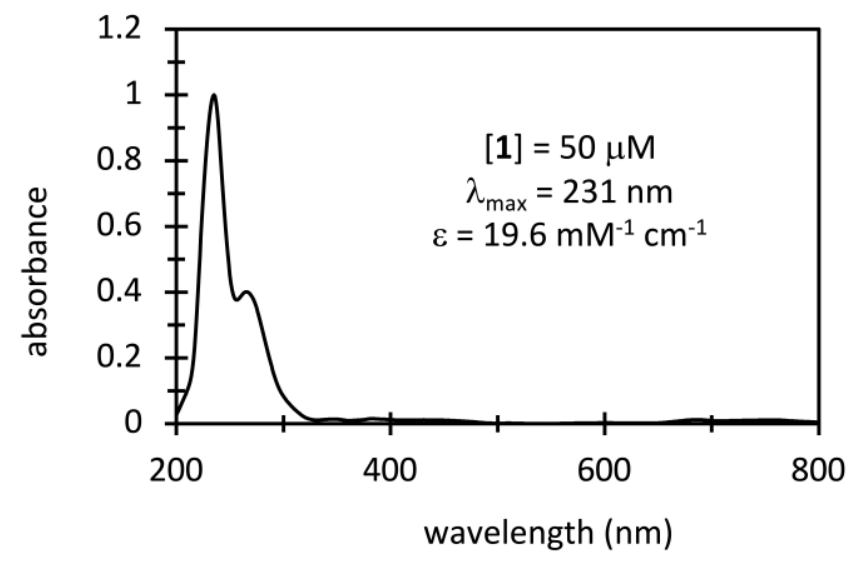

Figure S46. UV/Vis absorption spectrum of 2-nitrobenzyl benzoate (1).

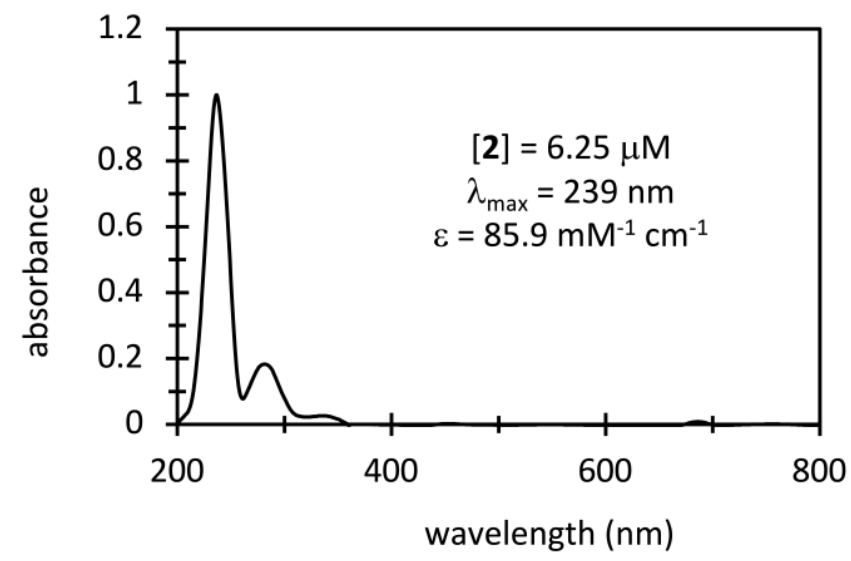

Figure S47. UV/Vis absorption spectrum of 2-nitrobenzyl 2-naphthoate (2). 


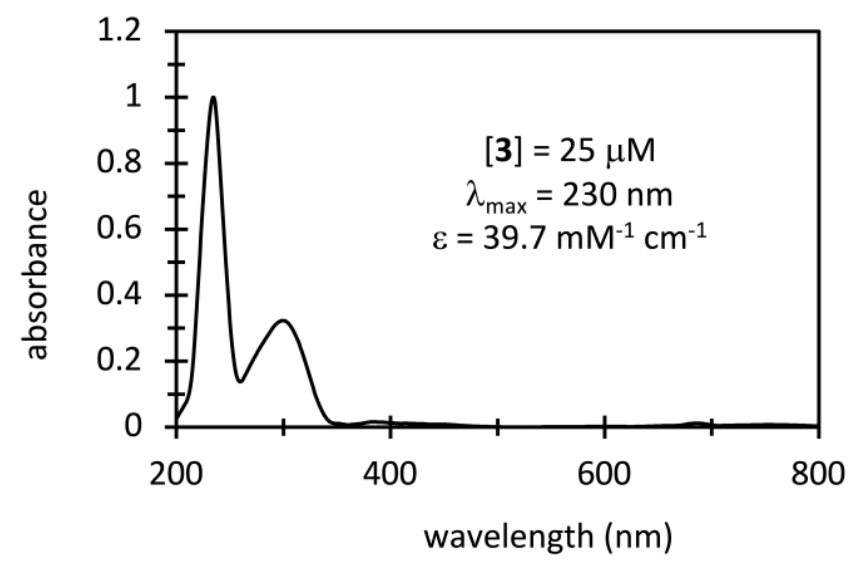

Figure S48. UV/Vis absorption spectrum of 2-nitrobenzyl 1-naphthoate (3).

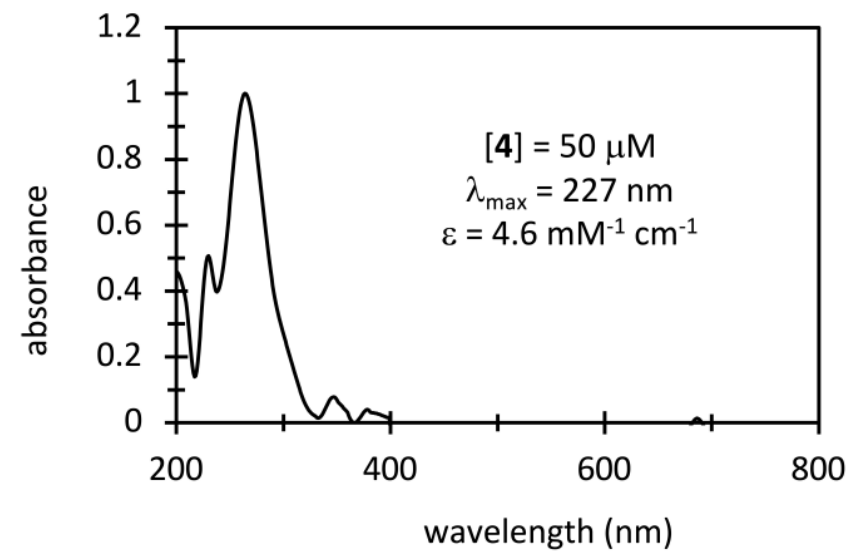

Figure S49. UV/Vis absorption spectrum of 2-nitrobenzyl 1-adamantane carboxylate (4).

For solid-state studies, melt-bonded samples of each material were prepared as described above. The bonded assemblies were then mounted in the analysis chamber of the spectrophotometer in place of a solution-filled cuvette. Each polycrystalline film was scanned from $\lambda=800-200 \mathrm{~nm}$ in transmission mode with a step size of $0.5 \mathrm{~nm}$ and a scan rate of $100 \mathrm{~nm}$ $\min ^{-1}$. Data were exported and analyzed in Origin Pro 2020. In the analysis, the transmission data was converted to give the percentage of incident light absorbed and/or scattered by the polycrystalline film, then normalized. The solution-state data from above was likewise normalized and overlaid on the solid-state data (Figures S50-S53). From these plots, it is evident that the majority of incident light in the range where the nitrobenzyl protecting groups absorb is 
scattered by the polycrystalline film. Consequently, the photochemical transformation is hypothesized to only occur as the adhesive-adherend interface and not throughout the bulk.

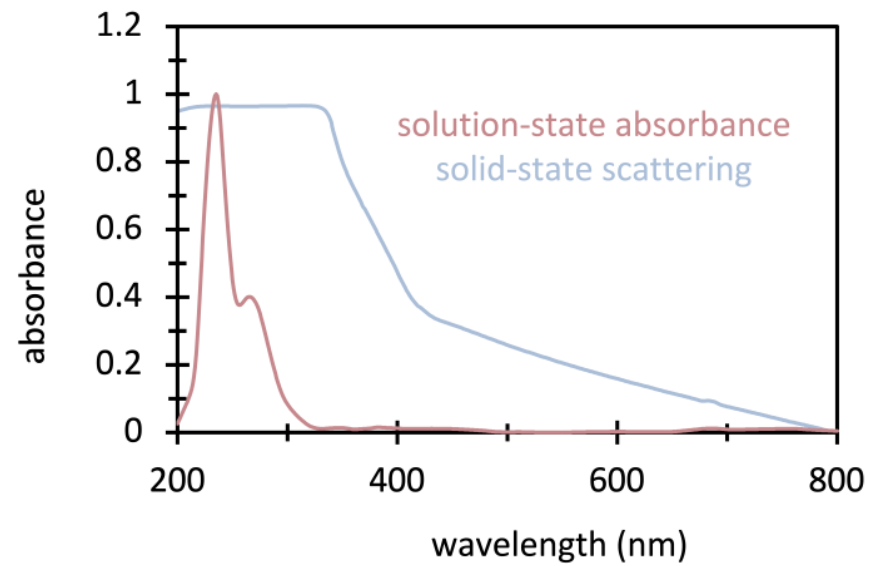

Figure S50. Solution- and solid-state UV/Vis spectra of 2-nitrobenzyl benzoate (1). In Solution, the material absorbs at $\lambda_{\max }=231 \mathrm{~nm}$. As a polycrystalline film, light scattering at the interface prevents transmission of UV light throughout the bulk of the adhesive.

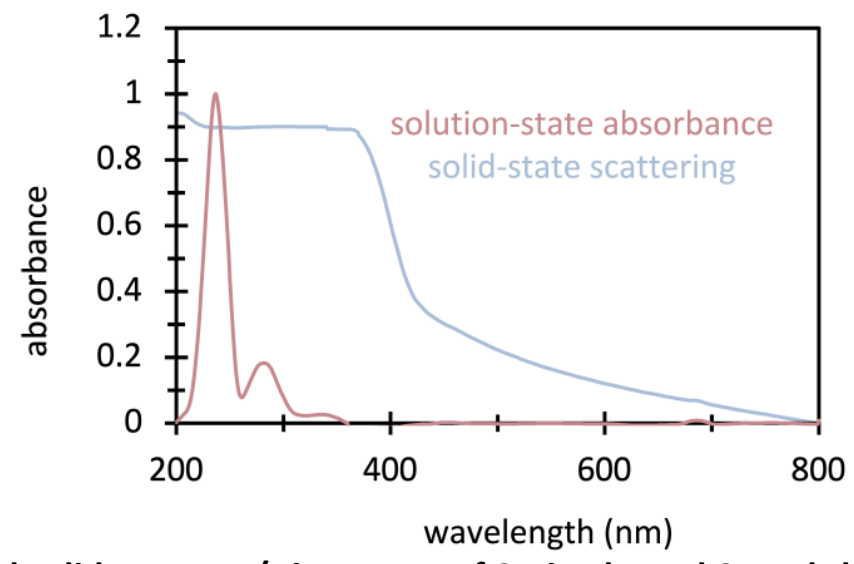

Figure S51. Solution- and solid-state UV/Vis spectra of 2-nitrobenzyl 2-naphthoate (2). In Solution, the material absorbs at $\lambda_{\max }=239 \mathrm{~nm}$. As a polycrystalline film, light scattering at the interface prevents transmission of UV light throughout the bulk of the adhesive. 


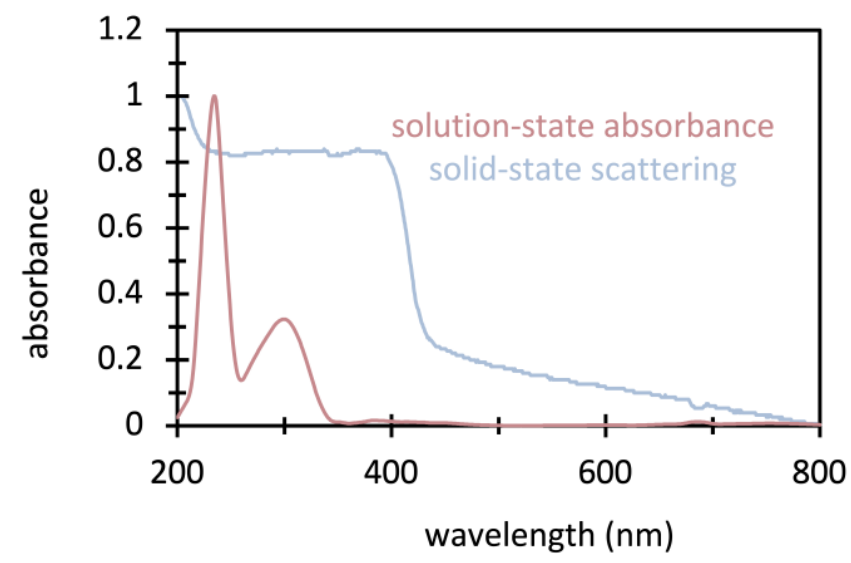

Figure S52. Solution- and solid-state UV/Vis spectra of 2-nitrobenzyl 1-naphthoate (3). In Solution, the material absorbs at $\lambda_{\max }=230 \mathrm{~nm}$. As a polycrystalline film, light scattering at the interface prevents transmission of UV light throughout the bulk of the adhesive.

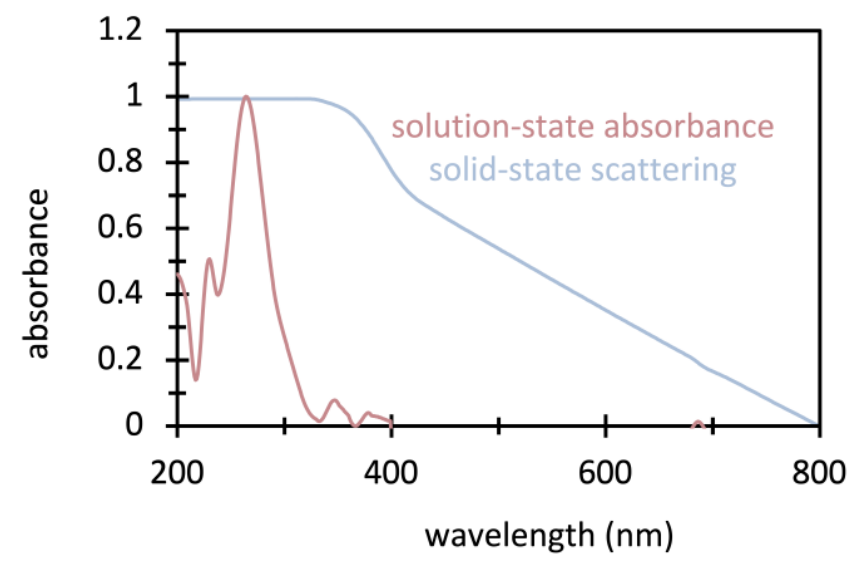

Figure S53. Solution- and solid-state UV/Vis spectra of 2-nitrobenzyl 1-adamantane carboxylate (4). In Solution, the material absorbs at $\lambda_{\max }=227 \mathrm{~nm}$. As a polycrystalline film, light scattering at the interface prevents transmission of UV light throughout the bulk of the adhesive.

\section{SXIII. Determination of the Reaction Rate for the Photolysis of Photoresponsive}

\section{Crystalline Adhesives}

Concentrated solutions of 1-4 in acetone-D6 were prepared in quartz NMR tubes. Each solution was exposed to broad spectrum UV radiation and the progress of the reaction was measured at selected time intervals (every 15 min or $1 \mathrm{~h}$ ) via NMR spectroscopy. An Ushio USH200DP Super High Pressure UV Lamp (200 W) housed in a Newport 67005 Arc Lamp Housing and powered by a Newport 69907 Universal Arc Lamp Power Supply was used as the light source. The 
reaction progress was calculated via integration of characteristic peaks for the starting material and the photoproduct. Once each reaction had gone to completion, the reaction progress at each time interval was plotted according to Equation 3 (Figures S54-S57). Reaction rates could be calculated by taking the negative of the slope of a line of best fit. Half-lives were calculated according to Equation 4.

$$
\begin{gathered}
f(t)=\ln \left(\frac{A}{A_{0}}\right) ; A \text { is }[\mathbf{1}-\mathbf{4}] \text { at time } t \text { and } A_{o} \text { is }[\mathbf{1}-\mathbf{4}] \text { at } t=0 \\
t_{1 / 2}=\frac{\ln (2)}{k} ; k \text { is the reaction rate }
\end{gathered}
$$

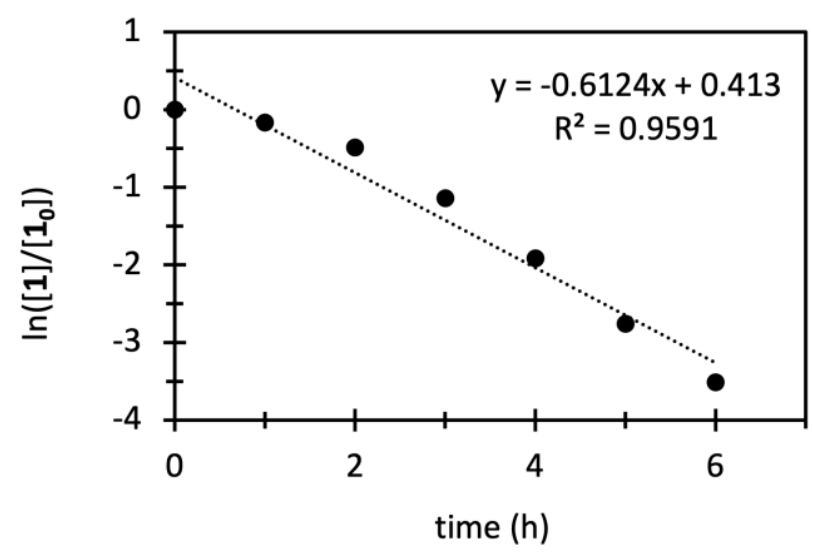

Figure S54. Progress of the photolysis of 2-nitrobenzyl benzoate (1) as monitored via NMR spectroscopy.

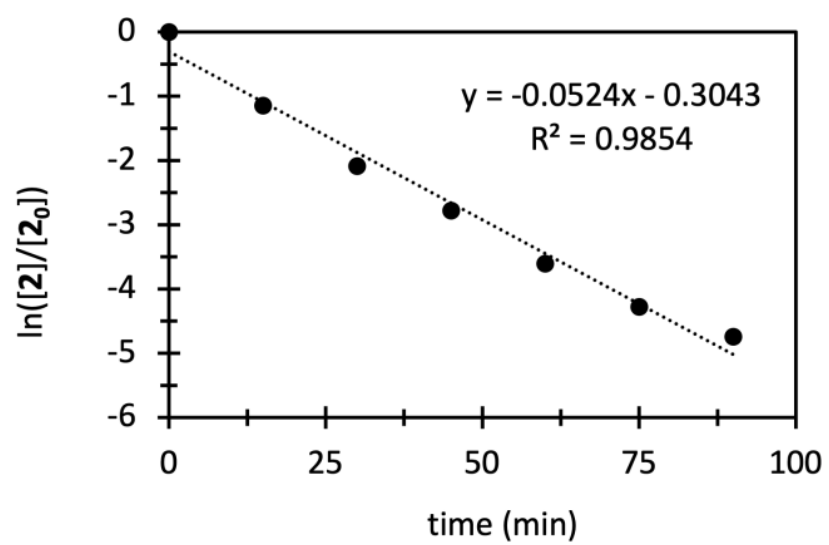

Figure S55. Progress of the photolysis of 2-nitrobenzyl 2-naphthoate (2) as monitored via NMR spectroscopy. 


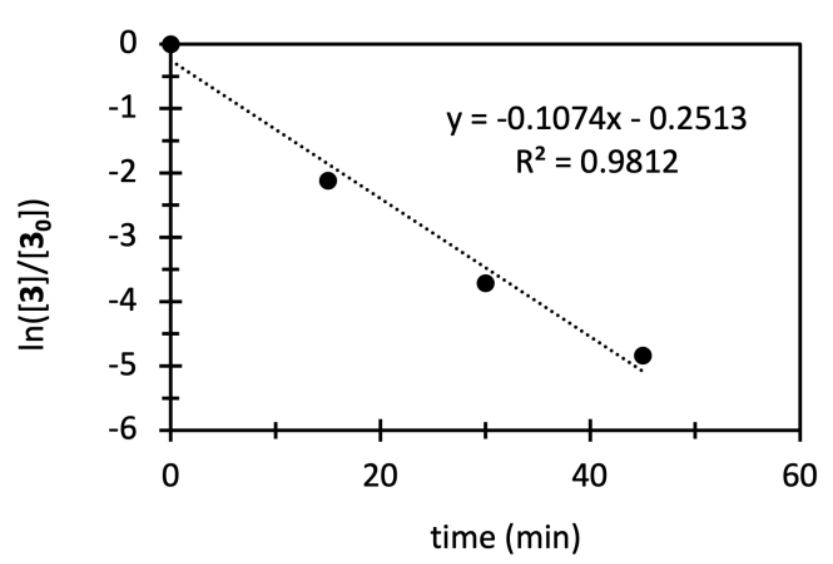

Figure S56. Progress of the photolysis of 2-nitrobenzyl 1-naphthoate (3) as monitored via NMR spectroscopy.

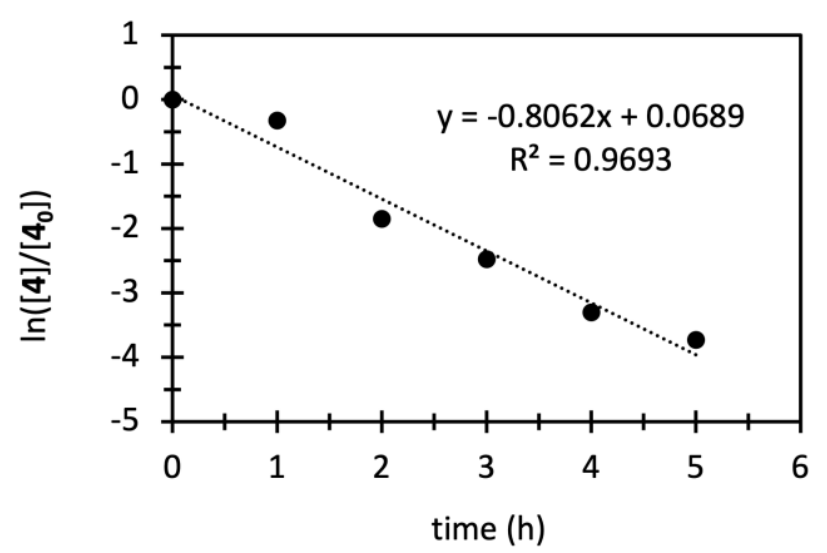

Figure S57. Progress of the photolysis of 2-nitrobenzyl 1-adamantane carboxylate (4) as monitored via NMR spectroscopy.

\section{SXIV. UV Irradiation of Quartz Assemblies Bonded with Photoresponsive}

\section{Crystalline Adhesives}

Ultraviolet (UV) exposure of 1-4 was achieved using a Hg arc lamp (Shanghai Jiguang, Supplier No.: GGZ400, 400 W, 3.24 A, 130 V) placed within a chemical fume hood lined with a reflective coating. Bonded samples were prepared as detailed above, then, during irradiation, were placed directly underneath the lamp to promote their optimal exposure. Real-time monitoring ensured that the ambient temperature never exceeded $25^{\circ} \mathrm{C}$, ensuring that all observed changes in mechanical and adhesive properties were due to UV irradiation and not thermal exposure. 
Melt-bonded samples were exposed in the solid state through either a "single exposure" or "double exposure" procedure. In the singe exposure process, a sample was exposed such that only one side of the overlap joint (i.e., one adhesive/adherend interface) was facing the UV light source. The total irradiation time was $3 \mathrm{~h}$. In the double exposure process, a sample was exposed such that one side of the overlap joint was facing the UV light source. After $3 \mathrm{~h}$ irradiation, the sample was then flipped such that the second side of the overlap joint was facing the UV light source. The second side was irradiated for another $3 \mathrm{~h}$, for a total of $6 \mathrm{~h}$ total irradiation. Mechanical testing of exposed samples was performed as detailed above.

\section{SXV. Solid-State Photolysis of Exposed Films of Photoresponsive Crystalline}

\section{Adhesives}

The changes in the crystallinity of exposed films (i.e., the polycrystalline film was exposed to the atmosphere and not covered by a quartz adherend) of materials 1-4 were monitored to visualize the interfacial intermolecular interactions that contribute to successful adhesion. In these experiments, bonded samples were prepared as detailed above. Once the sample had fully cooled and the adherend had fused, it was sheared by hand generating two quartz adherends with exposed films of adhesive material. The polycrystalline film that contained the majority of the adhesive material was analyzed via PXRD as described above. It was then subjected to broadspectrum UV irradiation (Ushio USH-200DP Super High Pressure UV Lamp, see SI Section SXIII for

specifications). The sample was analyzed via PXRD at regular time intervals for a total of 24 or $36 \mathrm{~h}$. 


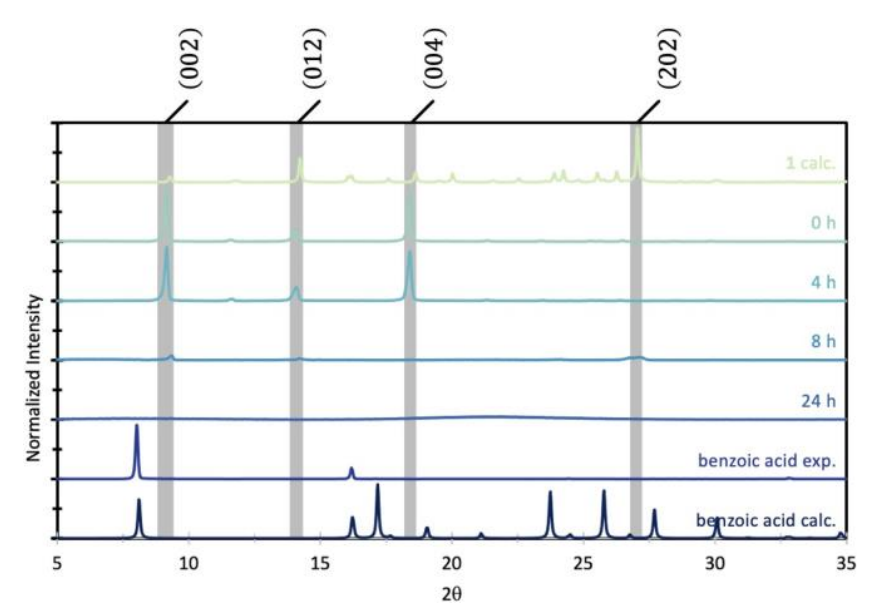

Figure S58. PXRD spectra of an exposed film of 2-nitrobenzyle benzoate (1) after UV irradiation.

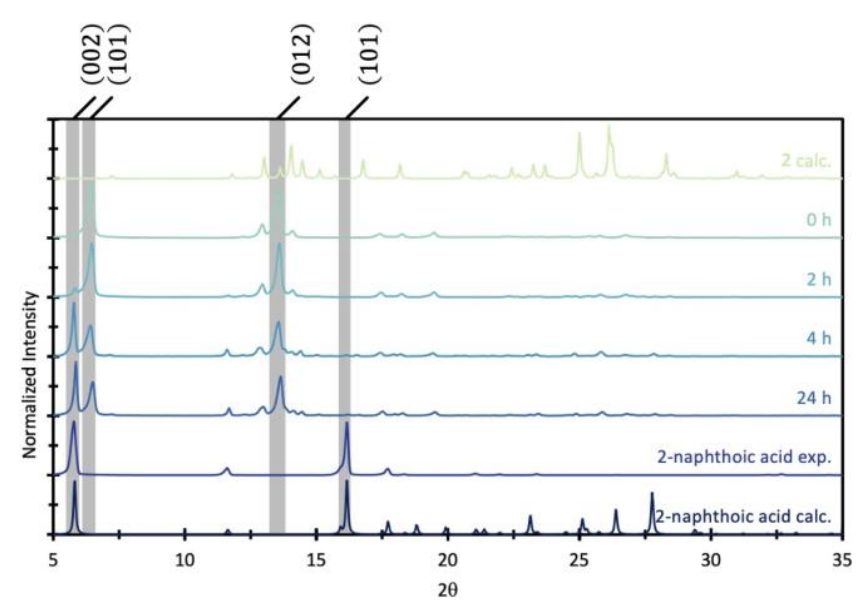

Figure S59. PXRD spectra of an exposed film of 2-nitrobenzyle 2-naphthoate (2) after UV irradiation.

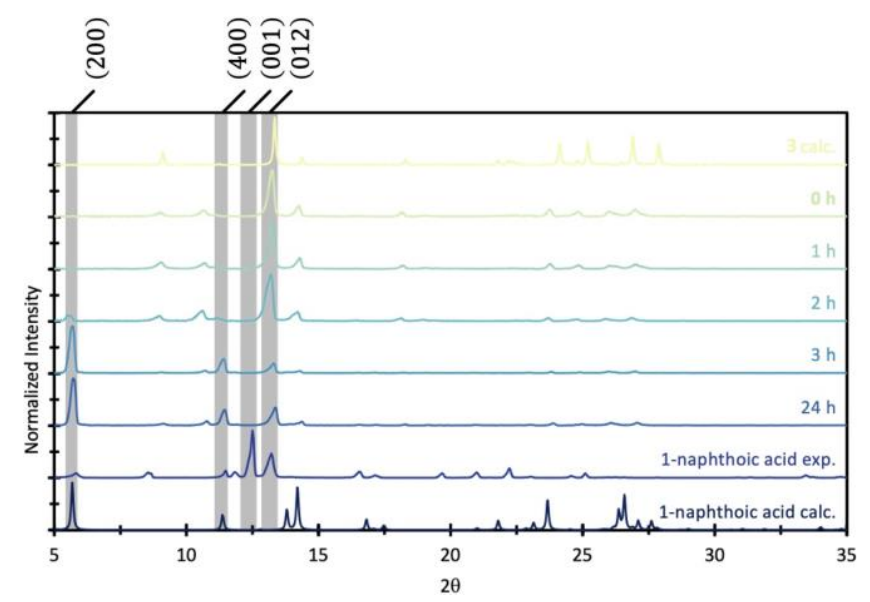

Figure S58. PXRD spectra of an exposed film of 2-nitrobenzyle 1-naphthoate (3) after UV irradiation. 


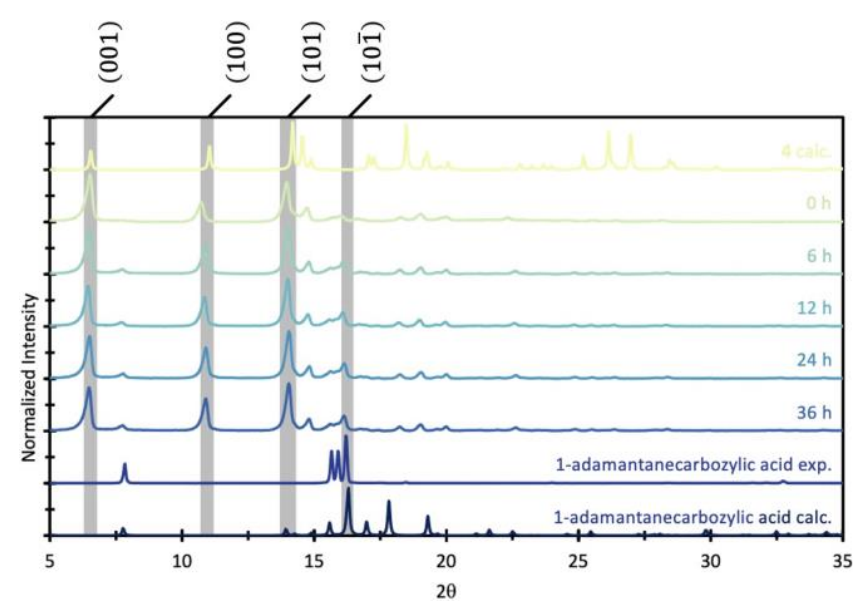

Figure S58. PXRD spectra of an exposed film of 2-nitrobenzyl 1-adamantane carboxylate (4) after UV irradiation.

\section{SXVI. Diffuse Reflectance Infrared Fourier Transform Spectroscopy of}

\section{Photoresponsive Crystalline Adhesives}

Diffuse Reflectance Infrared Fourier Transform Spectroscopy (DRIFTS) was performed in an attempt to characterize the solid-state photochemical transformation of materials 1-4. IR experiments were performed on a ThermoFisher Nicolet iS50 equipped with a DLaTGS detector and a Harrick Praying Mantis DRIFTS accessory in an open-cup configuration. Spectra were obtained over a range of $400-4000 \mathrm{~cm}^{-1}$ with a resolution of $4 \mathrm{~cm}^{-1}$ and integrated over 64 scans. $\mathrm{A} \mathrm{KBr}$ bean was subtracted from the sample beam to give double beam data. DRIFTS spectra were recorded for unexposed 1-4 and for the expected photoproducts (i.e., benzoic acid, 2-naphthoic acid, 1-naphthoic acid, and adamantane-1-carboxylic acid). Films of 1-4 were then prepared and irradiated (see SI Section SXV for details). The exposed material was then removed from the quartz slides and analyzed via DRIFTS. Significant overlap of diagnostic peaks in the DRIFTS spectra of 1-4 and the expected photoproducts limited valuable analysis (Figures S62-S65). 

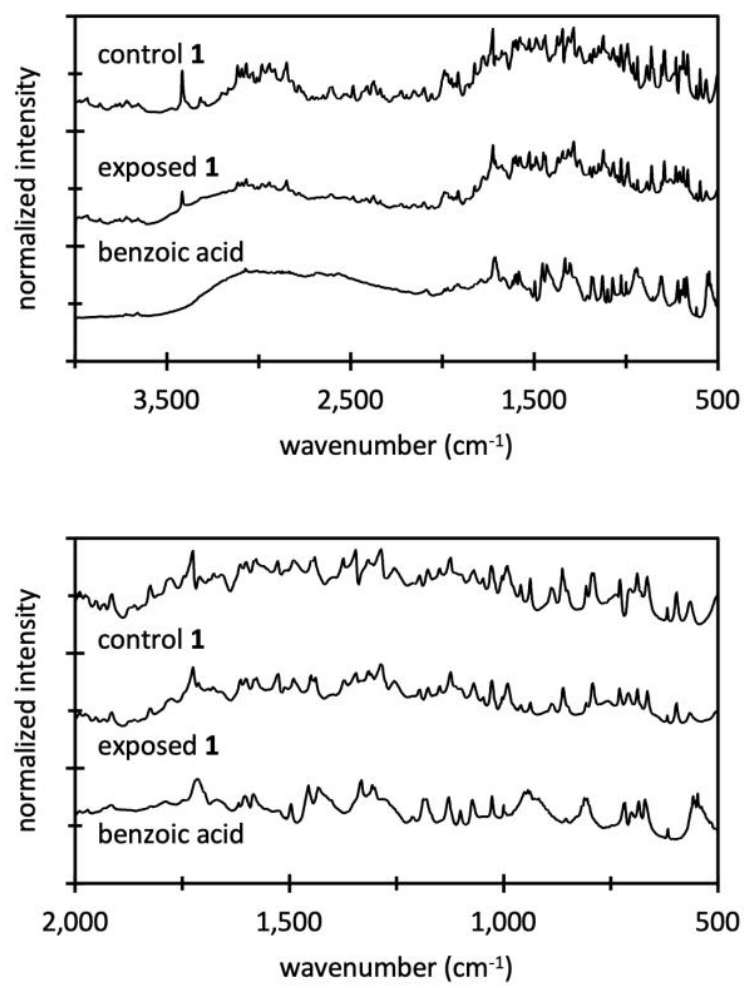

Figure S62. DRIFTS spectra of control and UV-irradiated 2-nitrobenzyl benzoate (1), and benzoic acid. 

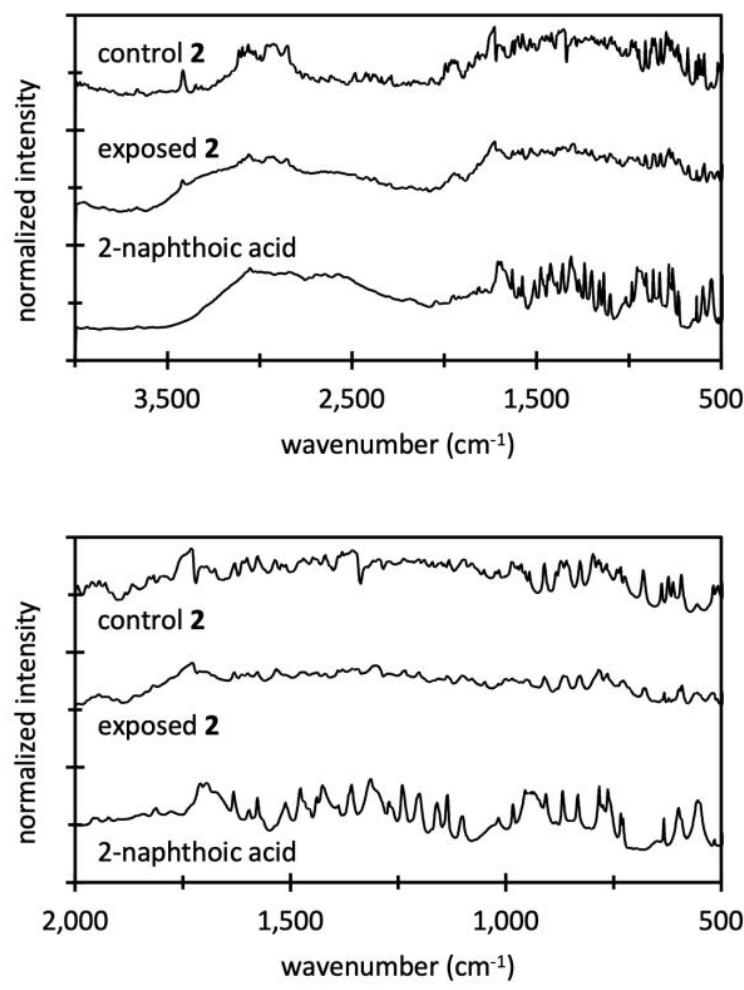

Figure S63. DRIFTS spectra of control and UV-irradiated 2-nitrobenzyl 2-naphthoate (2), and 2-naphthoic acid. 

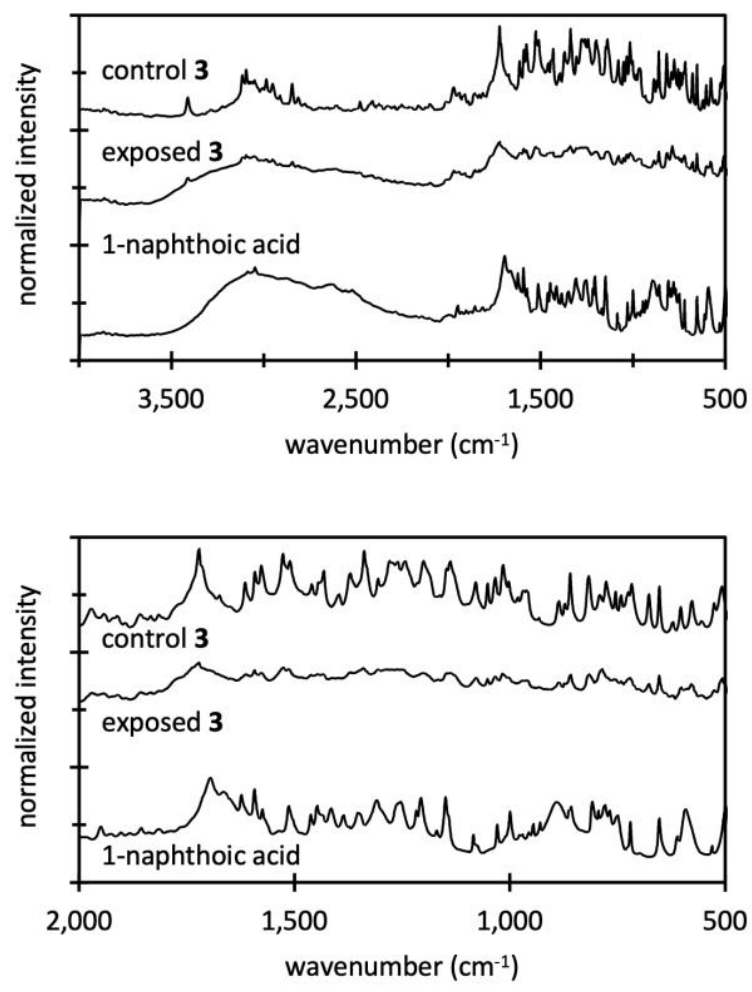

Figure S64. DRIFTS spectra of control and UV-irradiated 2-nitrobenzyl 1-naphthoate (3), and 1-naphthoic acid. 

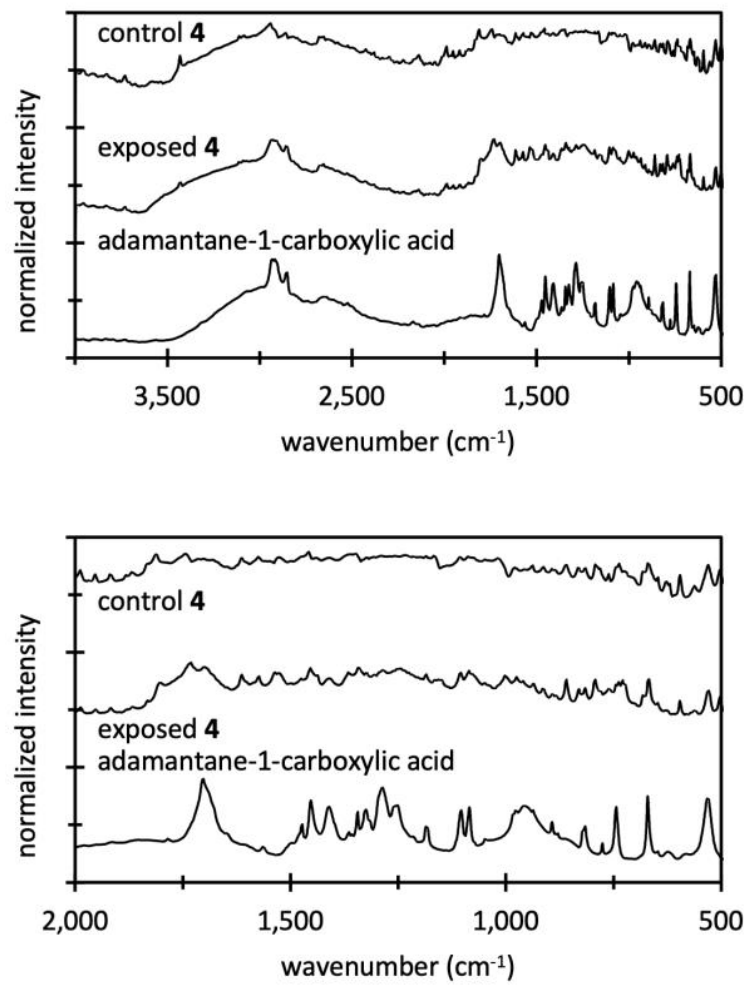

Figure S65. DRIFTS spectra of control and UV-irradiated 2-nitrobenzyl 1-adamantane carboxylate (4), and adamantane-1-carboxylic acid.

\section{SXVII. References}

(1) Bulatov, E.; Haukka, M. Non-Conventional Synthesis and Photophysical Studies of

Platinum(II) Complexes with Methylene Bridged 2,2'-Dipyridylamine Derivatives. Dalt.

Trans. 2019, 48 (10), 3369-3379. https://doi.org/10.1039/C8DT03912G.

(2) Dolomanov, O. V.; Bourhis, L. J.; Gildea, R. J.; Howarda, J. A. K.; Puschmanna, H. OLEX2: A Complete Structure Solution, Refinement and Analysis Program. J. Appl. Crystallogr. 2009, 42 (2), 339-341. https://doi.org/10.1107/S0021889808042726.

(3) Sheldrick, G. M. SHELXT - Integrated Space-Group and Crystal-Structure Determination. Acta Crystallogr. Sect. A Found. Adv. 2015, 71 (1), 3-8.

https://doi.org/10.1107/S2053273314026370. 
(4) Sheldrick, G. M. A Short History of SHELX. Acta Crystallogr. Sect. A 2008, 64 (1), 112-122. https://doi.org/10.1107/S0108767307043930.

(5) Groom, C. R.; Bruno, I. J.; Lightfoot, M. P.; Ward, S. C. The Cambridge Structural Database. Acta Crystallogr. Sect. B 2016, B72, 171-179.

https://doi.org/https://doi.org/10.1107/S2052520616003954.

(6) Turner, M. J.; McKinnon, J. J.; Wolff, S. K.; Grimwood, D. J.; Spackman, P. R.; Jayatilaka, D.; Spackman, M. A. Crystal Explorer. University of Western Australia 2020.

(7) Spackman, M. A.; McKinnon, J. J. Fingerprinting Intermolecular Interactions in Molecular Crystals. CrystEngComm 2002, 4 (66), 378-392. https://doi.org/10.1039/B203191B.

(8) McKinnon, J. J.; Spackman, M. A.; Mitchell, A. S.; IUCr. Novel Tools for Visualizing and Exploring Intermolecular Interactions in Molecular Crystals. Acta Crystallogr. Sect. B Struct. Sci. 2004, 60 (6), 627-668. https://doi.org/10.1107/S0108768104020300.

(9) McKinnon, J. J.; Jayatilaka, D.; Spackman, M. A. Towards Quantitative Analysis of Intermolecular Interactions with Hirshfeld Surfaces. Chem. Commun. 2007, 37, 3814. https://doi.org/10.1039/b704980c.

(10) Spackman, M. A.; Jayatilaka, D. Hirshfeld Surface Analysis. CrystEngComm 2009, 11 (1), 19-32. https://doi.org/10.1039/b818330a.

(11) Parkin, A.; Barr, G.; Dong, W.; Gilmore, C. J.; Jayatilaka, D.; McKinnon, J. J.; Spackman, M. A.; Wilson, C. C. Comparing Entire Crystal Structures: Structural Genetic Fingerprinting. CrystEngComm 2007, 9 (8), 648. https://doi.org/10.1039/b704177b.

(12) MATLAB. The MathWorks, Inc. 2020.

(13) E112-13 Standard Test Methods for Determineing Average Grain Size; West 
Conshohocken, PA, United States, 2013. https://doi.org/10.1520/E0112-13.

(14) Mitchell, H. T.; Smith, M. K.; Blelloch, N. D.; Van Citters, D. W.; Mirica, K. A. Polycyclic Aromatic Hydrocarbons as Sublimable Adhesives. Chem. Mater. 2017, 29 (7), 2788-2793. https://doi.org/10.1021/acs.chemmater.6b04641.

(15) Blelloch, N. D.; Mitchell, H. T.; Tymm, C. C.; Van Citters, D. W.; Mirica, K. A. Crystal Engineering of Molecular Solids as Temporary Adhesives. Chem. Mater. 2020, 32 (23), 9882-9896. https://doi.org/0.1021/acs.chemmater.0c01401.

(16) Standard Test Method for Apparent Shear Strength of Single-Lap-Joint Adhesively Bonded Metal Specimens by Tension Loading (Metal-to-Metal); West Conshohocken, PA, 2018. https://doi.org/10.1520/D1002-10.

(17) Mercury. Cambridge Crystallographic Data Centre 2020.

(18) Taylor, R.; Macrae, C. F.; IUCr. Rules Governing the Crystal Packing of Mono- and Dialcohols. Acta Crystallogr. Sect. B Struct. Sci. 2001, 57 (6), 815-827. https://doi.org/10.1107/S010876810101360X.

(19) Bruno, I. J.; Cole, J. C.; Edgington, P. R.; Kessler, M.; Macrae, C. F.; McCabe, P.; Pearson, J.; Taylor, R.; IUCr. New Software for Searching the Cambridge Structural Database and Visualizing Crystal Structures. Acta Crystallogr. Sect. B Struct. Sci. 2002, 58 (3), 389-397. https://doi.org/10.1107/S0108768102003324.

(20) Macrae, C. F.; Edgington, P. R.; McCabe, P.; Pidcock, E.; Shields, G. P.; Taylor, R.; Towler, M.; van de Streek, J.; IUCr. Mercury : Visualization and Analysis of Crystal Structures. J. Appl. Crystallogr. 2006, 39 (3), 453-457. https://doi.org/10.1107/S002188980600731X.

(21) Macrae, C. F.; Bruno, I. J.; Chisholm, J. A.; Edgington, P. R.; McCabe, P.; Pidcock, E.; 
Rodriguez-Monge, L.; Taylor, R.; van de Streek, J.; Wood, P. A.; et al. Mercury CSD 2.0 New Features for the Visualization and Investigation of Crystal Structures. J. Appl. Crystallogr. 2008, 41 (2), 466-470. https://doi.org/10.1107/S0021889807067908.

(22) Macrae, C. F.; Sovago, I.; Cottrell, S. J.; Galek, P. T. A.; McCabe, P.; Pidcock, E.; Platings, M.; Shields, G. P.; Stevens, J. S.; Towler, M.; et al. Mercury 4.0: From Visualization to Analysis, Design and Prediction. J. Appl. Crystallogr. 2020, 53 (1), 226-235. https://doi.org/10.1107/S1600576719014092. 